\title{
ETHNOBOTANICAL STUDY OF MEDICINAL PLANTS OF NAMAL VALLEY, SALT RANGE, PAKISTAN
}

\author{
SHAH, A. ${ }^{1}{ }^{*}$ - POUdEL, R. C. ${ }^{2}-$ ISHTIAQ, M. ${ }^{3}-$ SARVAT, R. ${ }^{1}-$ SHAHZAD, H. ${ }^{1}-$ ABBAS, A. ${ }^{1}-$ \\ SHOAIB, S. ${ }^{1}$ - NUZHAT, R. ${ }^{1}$ - NOOR, U. D. ${ }^{1}$ - MAHMOODA, H. ${ }^{1}$ - SUMmAYA, A. ${ }^{1}-$ IFRA, A. ${ }^{1}-$ \\ IHSAN, U. ${ }^{1}$ \\ ${ }^{1}$ Department of Botany, University of Sargodha, Sargodha-40100, Pakistan \\ ${ }^{2}$ Nepal Academy of Science and Technology, Pātan-44700, Nepal \\ ${ }^{3}$ Department of Botany, (Bhimber Campus), Mirpur University of Science \& Technology \\ Mirpur-10250 (AJK), Pakistan \\ *Corresponding author \\ e-mail: aminullah.amin@uos.edu.pk; phone:+92-48-923-0811-15ext.609
}

(Received $5^{\text {th }}$ Jan 2019; accepted $26^{\text {th }}$ Feb 2019)

\begin{abstract}
This paper presents the first quantitative ethnobotanical knowledge and practices of using native plants for different ailments from Namal Valley of Pakistan. Data was gathered by interviewing 350 informants through semi-structured questionnaires. A total of 217 taxa belonging to 166 genera and 70 families were documented. Fabaceae and Asteraceae families were found to be the most cited families (with 19 and 18 species receptively). Herbs represent the most cited life form (71\%) and flower was the most widely used part (34.8\%) with decoction as main mode of the utilization $(41.5 \%)$. On the basis of use values, the most commonly used ethnobotanical taxa in the Valley were reported to be Euphorbia heterophylla (0.7) and Merremia dissecta (0.6). The highest RFC value was noted for Aloe vera (0.14) while highest ICF value was estimated for dental problems category (0.7). Maximum similarity index was found in the studies with Bana Valley (JI 23.9). Similarity percentage of plants uses ranges from $0 \%$ to 15.7 (Bana Valley), while dissimilarity percentage varies up to $32.5 \%$ (Soon Valley). This study highlights the medicinal flora of study area that will serve as baseline for conservation and sustainable utilization through future research on bio prospecting of potential species to develop novel drugs.
\end{abstract}

Keywords: medicinal flora; conservation; Fabaceae; novel drugs; informant consensus factor

\section{Introduction}

Ethnobotanical studies on medicinal flora are crucial for developing novel drugs based on traditional knowledge of the local people (Heinrich and Gibbons, 2001; Mesfin et al., 2009; Vitalini et al., 2013). The usage of medicinal plants for medicinal and therapeutic purposes has been studied and documented globally (Kunwar et al., 2015; Bulut et al., 2017; Fortini et al., 2016; Menale et al., 2016; Shah and Rahim, 2017). The role of herbal treatment in curing various diseases is vital because of the fact that a large number of ailments are cured by plant based medications (Rehecho et al., 2011). In this context ethnobotanical researches demonstrate high significance and prominence of medicinal plants in cognitive pluralistic perspective. Such studies not only play an important role in upgrading the social status and economic values of an area but also preserve the aboriginal medico-ethnobotanical data of the indigenous communities that consequently preserve the global heritage (Sanz-Biset et al., 2009). Medicinal plants are considered very important among the rural communities due to their potential to cure health related problems for which several of remote communities are unreachable of modern health care facilities (Heinrich, 2000; Tabuti et al., 2003; Verma and Singh, 2008). Out of approximately 6000 plants species in Pakistan 600 to 700 are reported to have medicinal value. Among them 456 plants species are used in 
the formulation of approximately 350 synthetic drugs (Ahmad and Husain, 2008). Pakistan has variations on climatic conditions, ecological zones and topography where diverse flora of medical importance flourishes in dry to temperate habitats. Limited number of ethnobotanical expeditions have been conducted in well-known valleys of Pakistan (Ahmad et al., 2014; Amjad, 2015; Bano et al., 2014; Haidar and Qaiser, 2009; Haq, 2012; Hazrat et al., 2011; Khan et al., 2010; Khan et al., 2013; Khan and Khatoon, 2008; Shah and Rahim, 2017; Zabihullah et al., 2006) but most of the remote valleys are still unexplored There could be hundreds of plant species growing in these valleys having medicinal, industrial and economic potential. Local communities of various regions use these species in different ways on the basis of their cultural beliefs and inherited experience. However, little attention is being paid to the conservation and sustainable utilization of several of potential species. Salt Range of Pakistan is one of such region which is least explored regarding the documentation of medicinal wealth present in several of its valleys. Namal Valley is among the most fascinating area awaiting systematic study of its ethnobotanical knowledge associated with native flora of the region. Recently (Shah et al., 2018) has documented sixty eight (68) medicinal plants used in snakebite and scorpion sting used by the inhabitants of this valley who are predominantly pastoralists, peasants and farmers. Namal Valley is naturally gifted with diverse flora with social, economic and environmental importance. The objectives of this study were to collect record and document information regarding the plants used ethnobotanically by the aboriginal people especially with respect to the medicinal wealth of the plants. This extensive study carried out throughout the Valley revealed an exhaustive list profile of medicinal plants, based on quantitative evaluations adopting several ethnobotanical indices like use value (UV), frequency of citation (FC), relative frequency citation (RFC), family importance value (FIV), informant consensus factor (ICF) and the Jaccard index (JI).

\section{Material and Methods}

\section{Study area}

Geographically, the study area was located in the northwest of Punjab Province geographically ranging at $71^{\circ} 48^{\circ} 45^{\circ} \mathrm{E}$ longitude and $32^{\circ} 40^{\circ} 10^{\circ} \mathrm{N}$ latitude, spreading over an area of 5.5 square kilometers. The valley is bounded by Salt Range Mountains and touches western border of Mianwali district (Fig. 1). Historical and scenic places of the Valley include Namal College Mianwali, Namal Dam, Namal Lake, Sulphur spring and shrines of Hafiz Jee and Khaki Shah. Namal valley is included among the oldest civilization in Pakistan. Raring of cattle and goats provides a livelihood for the local people Most part of the Valley is covered by forests and pastures The climate of the Valley is characterized by cold, dry weather at high altitudes and humid, warm in low altitude-lying areas. Several native tribes with a rich historical background and that use the native medicinal flora and have their own traditional healers known as tabeeb or hakeem are in the Valley. These tribes are awan, malik, niazi, shah, mian etc. Climate variation and complex topography gives way to diverse flora and fauna in the Valley. Valley is carpeted with Dodonaea viscosa, Prosopis glandulosa, P. juliflora, Tamarix aphylla, T. dioica, Tephrosia pupurea, Withania coagulans, Pluchea arabica, Pulicaria glutinosa and Rhazya stricta in the low altitudes while Acacia modesta mixed with Salvadora oleoides are commonly seen in high altitudes. Viola cinerea and Pseudogaillonia hymnostephana are among the rare taxa of the Valley that have also rare occurrence not only from the Pakistan but also globally (Shah et al., 2018). 


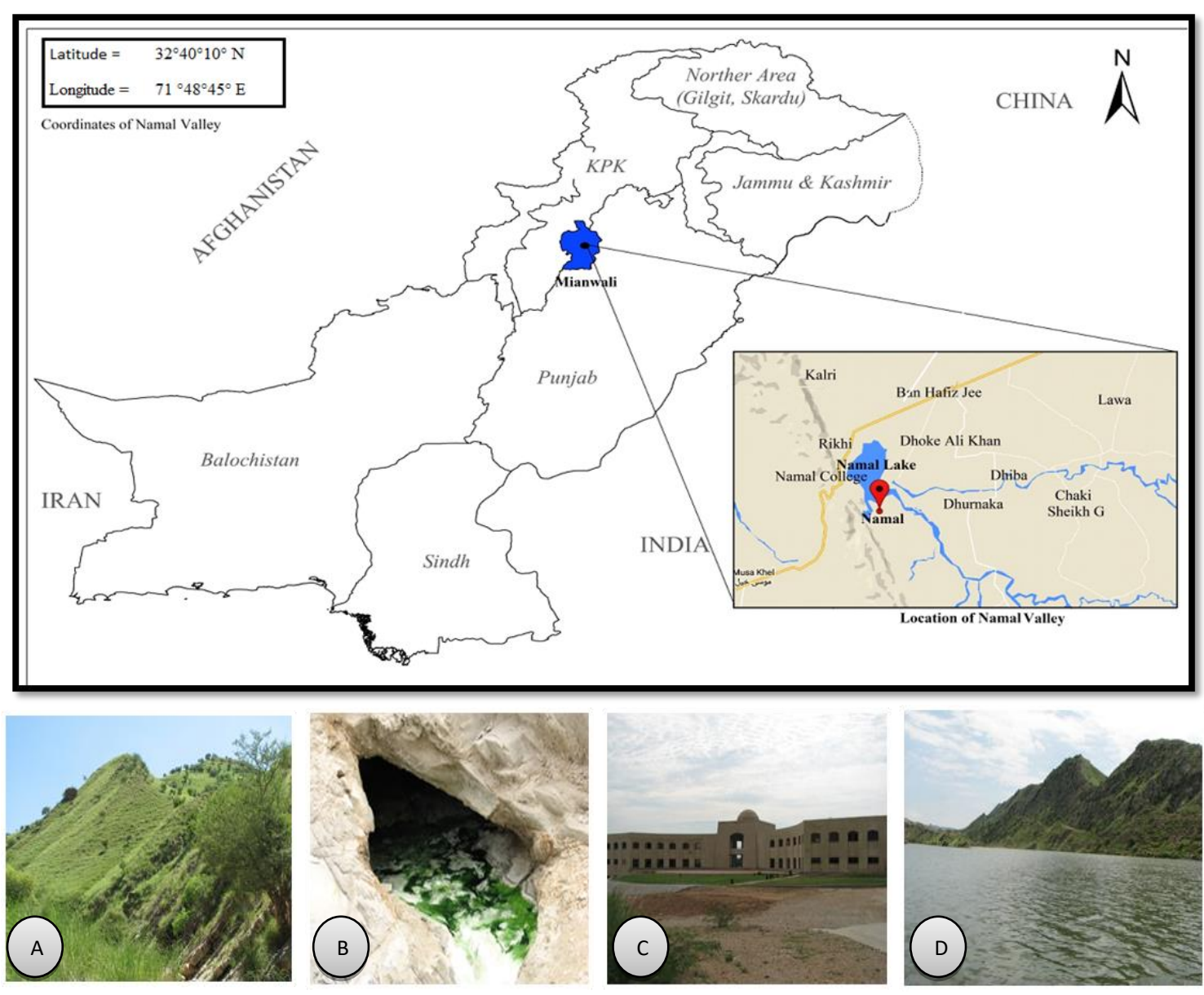

Figure 1. Map and Panoramic views of the Namal Valley

(A- Lush green scenic Valley hills; B-Sulphur Spring; C-Namal college; D- Namal Lake)

\section{Ethnobotanical data collection}

Data were collected from all the villages of the Valley from 2013-2016 representing all the seasons following the method of (Martin, 1985; Heinrich et al., 2009). A total of 16 field trips (representing all seasons) were carried out to collect plants and to document ethnobotanical information. Keeping in view the aim of quantitative approach to record ethnobotanical information, the participant observation method was used along with open-ended interviews and questionnaires. Knowledgeable people of the Valley including 12 traditional healers, shepherds, herbal medicinal venders, were consulted. The people in the local area speak Saraiki language and therefore, interviews were conducted in Saraiki language. All the documented data was later translated into English. A total of 350 informants ( 240 males and 110 females) of all group ages (25 to $\geq 65$ ) were interviewed. Information regarding vernacular names, ethnobotanical uses etc. was recorded and the details are presented in Table 1. 
Table 1. Ethnobotanical uses of plants in Namal Valley, Salt Range, Pakistan

\begin{tabular}{|c|c|c|c|c|c|c|c|c|c|c|}
\hline $\begin{array}{l}\text { Sr. } \\
\text { No. }\end{array}$ & $\begin{array}{c}\text { Species/ Family } \\
\text { Name/ Vernacular } \\
\text { Name/ Voucher } \\
\text { number } \\
\end{array}$ & $\begin{array}{l}\text { Habit } \\
\text { /Type }\end{array}$ & $\begin{array}{l}\text { Part(s) used/ } \\
\text { Mode of } \\
\text { utilization }\end{array}$ & $\begin{array}{l}\text { Medicinal Value/ } \\
\text { Disease treated }\end{array}$ & $\mathbf{U R}^{*}$ & $\mathbf{U V}^{*}$ & FC $^{*}$ & RFC $^{*}$ & $\begin{array}{l}\text { Previous reports } \\
\text { for comparison** }\end{array}$ & Picture \\
\hline 1 & $\begin{array}{l}\text { Abutilon indicum } \\
\text { (L.) Sweet/ } \\
\text { Malvaceae } \\
\text { SAN-SR-06 }\end{array}$ & $\begin{array}{c}\text { Shrub } \\
\text { / W }\end{array}$ & $\begin{array}{c}\text { Flower, leaves/ } \\
\text { Decoction }\end{array}$ & $\begin{array}{c}\text { Respiratory disorders, } \\
\text { menstrual disorders }\end{array}$ & 2 & 0.2 & 10 & 0.03 & $\begin{array}{c}1^{*}, 2^{*}, 3^{*}, 4^{*}, 5^{*}, \\
6^{*}, 7^{*}, 8^{*}, 9^{*}, 10^{*}, \\
11^{*}, 12^{*}, 13^{*}, 14^{*}, \\
15^{*}, 16^{*}, 17^{*}, 18^{*}, \\
19^{*}, 20^{*}, 21^{*}, 22^{*}, \\
23^{*}\end{array}$ & \\
\hline 2 & $\begin{array}{c}\text { Acacia modesta } \\
\text { Wall./ Fabaceae } \\
\text { Phulahi } \\
\text { SAN-SR-55 }\end{array}$ & $\begin{array}{c}\text { Tree/ } \\
\mathrm{W}\end{array}$ & $\begin{array}{l}\text { Twigs, pods/ } \\
\text { Raw, } \\
\text { decoction }\end{array}$ & $\begin{array}{l}\text { Gummosis, miswak } \\
\text { sticks, toothache, } \\
\text { diarrhea, Expectorant, } \\
\text { Gynaecological } \\
\text { problems }\end{array}$ & 6 & 0.17 & 35 & 0.1 & $\begin{array}{c}1^{*}, 2^{\star}, 3^{\bullet}, 4^{*}, 5^{\star} \\
6^{*}, 7^{*}, 8^{\bullet}, 9^{*}, 10^{*}, \\
11^{*}, 12^{*}, 13^{*}, 14^{*}, \\
15^{*}, 16^{*}, 17^{*}, 18^{*}, \\
19^{*}, 20^{*}, 21^{*}, 22^{*}, \\
2^{*}\end{array}$ & \\
\hline 3 & $\begin{array}{c}\text { Acacia nilotica }(\text { L.) } \\
\text { Delile./ Fabaceae } \\
\text { Kikar, Babool } \\
\text { SAN-SR-64 }\end{array}$ & $\begin{array}{c}\text { Tree/ } \\
\text { W }\end{array}$ & $\begin{array}{l}\text { Twigs, pods / } \\
\text { Raw, } \\
\text { decoction }\end{array}$ & $\begin{array}{l}\text { Gummosis, miswak } \\
\text { sticks, toothache, } \\
\text { Expectorant, } \\
\text { Gynaecological } \\
\text { problems }\end{array}$ & 5 & 0.2 & 25 & 0.07 & $\begin{array}{c}1^{\bullet}, 2^{*}, 3^{\bullet}, 4^{*}, 5^{\mathbf{}} \\
6^{*}, 7^{*}, 8^{\star}, 9^{*}, 10^{*}, \\
11^{*}, 12^{*}, 13^{\bullet}, 14^{*}, \\
15^{*}, 16^{*}, 17^{*}, 18^{*}, \\
19^{*}, 20^{*}, 21^{*}, 22^{*}, \\
23^{*}\end{array}$ & \\
\hline
\end{tabular}




\begin{tabular}{|c|c|c|c|c|c|c|c|c|c|c|}
\hline $\begin{array}{l}\text { Sr. } \\
\text { No. }\end{array}$ & $\begin{array}{c}\text { Species/ Family } \\
\text { Name/ Vernacular } \\
\text { Name/ Voucher } \\
\text { number } \\
\end{array}$ & $\begin{array}{l}\text { Habit } \\
\text { /Type }\end{array}$ & $\begin{array}{l}\text { Part(s) used/ } \\
\text { Mode of } \\
\text { utilization }\end{array}$ & $\begin{array}{l}\text { Medicinal Value/ } \\
\text { Disease treated }\end{array}$ & $\mathbf{U R}^{*}$ & $\mathbf{U V}^{*}$ & $\mathbf{F C}^{*}$ & RFC $^{*}$ & $\begin{array}{l}\text { Previous reports } \\
\text { for comparison** }\end{array}$ & Picture \\
\hline 4 & $\begin{array}{c}\text { Achyranthes aspera } \\
\text { L./ Amaranthaceae } \\
\text { Puthkanda } \\
\text { SAN-SR-45 }\end{array}$ & $\begin{array}{l}\text { Herb/ } \\
\text { W }\end{array}$ & $\begin{array}{l}\text { Whole plant/ } \\
\text { Decoction }\end{array}$ & $\begin{array}{l}\text { Cough, cold, febrifuge, } \\
\text { menstrual problems, } \\
\text { kidney pain }\end{array}$ & 5 & 0.42 & 12 & 0.03 & $\begin{array}{c}1^{*}, 2^{*}, 3^{*}, 4^{\star}, 5^{*}, 6^{*}, \\
7^{*}, 8^{\bullet}, 9^{*}, 10^{*}, 11^{\bullet} \\
12^{*}, 13^{*}, 14^{*}, 15^{*} \\
16^{*}, 17^{\bullet}, 18^{*}, 19^{*} \\
20^{*}, 21^{*}, 22^{*}, 23^{\star}\end{array}$ & \\
\hline 5 & $\begin{array}{l}\text { Adiantum capillus- } \\
\text { veneris L./ Pteridaceae } \\
\text { Paersichhayon } \\
\text { SAN-SR-33 }\end{array}$ & $\begin{array}{l}\text { Herb/ } \\
\text { W }\end{array}$ & $\begin{array}{l}\text { Whole plant/ } \\
\text { Decoction }\end{array}$ & $\begin{array}{l}\text { Hair tonic, febrifuge, } \\
\text { respiratory problems }\end{array}$ & 3 & 0.43 & 7 & 0.02 & $\begin{array}{c}1^{*}, 2^{*}, 3^{*}, 4^{*}, 5^{*}, 6^{*}, \\
7^{*}, 8^{*}, 9^{*}, 10^{*}, 11^{*} \\
12^{*}, 13^{*}, 14^{*}, 15^{*} \\
16^{*}, 17^{*}, 18^{*}, 19^{*} \\
20^{*}, 21^{*}, 22^{*}, 23^{*}\end{array}$ & \\
\hline 6 & $\begin{array}{c}\text { Aerva javanica } \\
\text { (Burm.f.) Juss. ex } \\
\text { Schult./ } \\
\text { Amaranthaceae } \\
\text { Bui } \\
\text { SAN-SR-91 }\end{array}$ & $\begin{array}{l}\text { Herb/ } \\
\text { W }\end{array}$ & $\begin{array}{l}\text { Leaves/ } \\
\text { Decoction }\end{array}$ & $\begin{array}{l}\text { Skin problems, joint } \\
\text { pain, vomiting, eye } \\
\text { infection }\end{array}$ & 4 & 0.2 & 20 & 0.06 & $\begin{array}{c}1^{*}, 2^{*}, 3^{*}, 4^{*}, 5^{*}, 6^{*} \\
7^{*}, 8^{*}, 9^{*}, 10^{*}, 11^{*} \\
12^{*}, 13^{*}, 14^{*}, 15^{*} \\
16^{*}, 17^{*}, 18^{*}, 19^{*} \\
20^{*}, 21^{*}, 22^{*}, 23^{\star}\end{array}$ & \\
\hline 7 & $\begin{array}{c}\text { Ageratum conyzoides } \\
\text { (L.) L. / Asteraceae } \\
\text { Osarri } \\
\text { SAN-SR-109 }\end{array}$ & $\begin{array}{l}\text { Herb/ } \\
\text { W }\end{array}$ & $\begin{array}{l}\text { Whole plant/ } \\
\text { Decoction, juice }\end{array}$ & $\begin{array}{l}\text { Skin problems, fever, } \\
\text { cough }\end{array}$ & 3 & 0.2 & 15 & 0.04 & $\begin{array}{c}1^{*}, 2^{*}, 3^{*}, 4^{*}, 5^{*}, 6^{*}, \\
7^{*}, 8^{*}, 9^{\star}, 10^{*}, 11^{*} \\
12^{*}, 13^{*}, 14^{*}, 15^{*} \\
16^{*}, 17^{*}, 18^{*}, 19^{*} \\
20^{*}, 21^{*}, 22^{*}, 23^{*}\end{array}$ & \\
\hline
\end{tabular}

APPLIED ECOLOGY AND ENVIRONMENTAL RESEARCH 17(2):4725-4805.

http://www.aloki.hu • ISSN 15891623 (Print) • ISSN 17850037 (Online)

DOI: http://dx.doi.org/10.15666/aeer/1702_47254805

(c) 2019, ALÖKI Kft., Budapest, Hungary 


\begin{tabular}{|c|c|c|c|c|c|c|c|c|c|c|}
\hline $\begin{array}{l}\text { Sr. } \\
\text { No. }\end{array}$ & $\begin{array}{c}\text { Species/ Family } \\
\text { Name/ Vernacular } \\
\text { Name/ Voucher } \\
\text { number } \\
\end{array}$ & $\begin{array}{l}\text { Habit } \\
\text { /Type }\end{array}$ & $\begin{array}{l}\text { Part(s) used/ } \\
\text { Mode of } \\
\text { utilization }\end{array}$ & $\begin{array}{l}\text { Medicinal Value/ } \\
\text { Disease treated }\end{array}$ & $\mathbf{U} \mathbf{R}^{*}$ & $\mathbf{U V}^{*}$ & FC $^{*}$ & RFC $^{*}$ & $\begin{array}{l}\text { Previous reports } \\
\text { for comparison** }\end{array}$ & Picture \\
\hline 8 & $\begin{array}{c}\text { Ajuga bracteosa Wall. } \\
\text { ex Benth./ Lamiaceae } \\
\text { SAN-SR-75 }\end{array}$ & $\begin{array}{l}\text { Herb/ } \\
\text { W }\end{array}$ & $\begin{array}{l}\text { Whole plant/ } \\
\text { Decoction }\end{array}$ & $\begin{array}{l}\text { Malaria fever, vomiting, } \\
\text { headache }\end{array}$ & 3 & 0.43 & 7 & 0.02 & 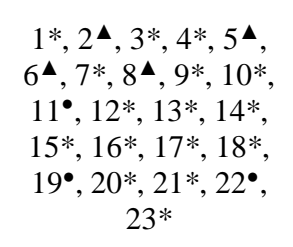 & \\
\hline 9 & $\begin{array}{c}\text { Albizia lebbeck (L.) } \\
\text { Benth../ } \\
\text { Fabaceae } \\
\text { Kala Shrin } \\
\text { SAN-SR-145 }\end{array}$ & $\begin{array}{l}\text { Tree/ } \\
\text { W,C }\end{array}$ & $\begin{array}{l}\text { Leaves/ } \\
\text { Decoction }\end{array}$ & $\begin{array}{c}\text { Cardio tonic, diarrhea. } \\
\text { Aphrodisiac, skin } \\
\text { problems, }\end{array}$ & 4 & 0.16 & 25 & 0.07 & $\begin{array}{c}1^{*}, 2^{*}, 3^{*}, 4^{*}, 5^{*}, 6^{*}, \\
7^{*}, 8^{*}, 9^{*}, 10^{*}, 11^{*}, \\
12^{*}, 13^{*}, 14^{*}, 15^{*} \\
16^{*}, 17^{*}, 18^{*}, 19^{*} \\
20^{*}, 21^{*}, 22^{*}, 23^{*}\end{array}$ & \\
\hline 10 & $\begin{array}{c}\text { Albizia procera } \\
\text { (Roxb.) Benth./ } \\
\text { Fabaceae } \\
\text { Safed shrin } \\
\text { SAN-SR-139 }\end{array}$ & $\begin{array}{l}\text { Tree } \\
\text { W,C }\end{array}$ & $\begin{array}{l}\text { Leaves/ } \\
\text { Decoction }\end{array}$ & $\begin{array}{c}\text { Inflammation, menstrual } \\
\text { problems, Hepatic pain } \\
\text { epilepsy }\end{array}$ & 4 & 0.11 & 35 & 0.1 & $\begin{array}{c}1^{*}, 2^{*}, 3^{*}, 4^{*}, 5^{*}, 6^{*} \\
7^{*}, 8^{*}, 9^{*}, 10^{*}, 11^{*} \\
12^{*}, 13^{*}, 14^{*}, 15^{*} \\
16^{*}, 17^{*}, 18^{*}, 19^{*} \\
20^{*}, 21^{*}, 22^{*}, 23^{*}\end{array}$ & \\
\hline 11 & $\begin{array}{c}\text { Alhagi maurorum } \\
\text { Medik./ Fabaceae } \\
\text { Oont katara } \\
\text { SAN-SR-128 }\end{array}$ & $\begin{array}{l}\text { Shrub/ } \\
\text { W }\end{array}$ & Pods, leaves & Piles, laxative, obesity & 3 & 0.5 & 6 & 0.02 & $\begin{array}{c}1^{*}, 2^{*}, 3^{*}, 4^{*}, 5^{*}, 6^{*} \\
7^{*}, 8^{*}, 9^{*}, 10^{*}, 11^{*} \\
12^{*}, 13^{*}, 14^{*}, 15^{*} \\
16^{*}, 17^{*}, 18^{*}, 19^{*} \\
20^{*}, 21^{*}, 22^{*}, 23^{*}\end{array}$ & \\
\hline
\end{tabular}

APPLIED ECOLOGY AND ENVIRONMENTAL RESEARCH 17(2):4725-4805.

http://www.aloki.hu • ISSN 15891623 (Print) • ISSN 17850037 (Online)

DOI: http://dx.doi.org/10.15666/aeer/1702_47254805

(c) 2019, ALÖKI Kft., Budapest, Hungary 


\begin{tabular}{|c|c|c|c|c|c|c|c|c|c|c|}
\hline $\begin{array}{l}\text { Sr. } \\
\text { No. }\end{array}$ & $\begin{array}{c}\text { Species/ Family } \\
\text { Name/ Vernacular } \\
\text { Name/ Voucher } \\
\text { number } \\
\end{array}$ & $\begin{array}{l}\text { Habit } \\
\text { /Type }\end{array}$ & $\begin{array}{l}\text { Part(s) used/ } \\
\text { Mode of } \\
\text { utilization }\end{array}$ & $\begin{array}{l}\text { Medicinal Value/ } \\
\text { Disease treated }\end{array}$ & $\mathbf{U R}^{*}$ & $\mathbf{U V}^{*}$ & $\mathbf{F C}^{*}$ & RFC $^{*}$ & $\begin{array}{l}\text { Previous reports } \\
\text { for comparison }^{* *}\end{array}$ & Picture \\
\hline 12 & $\begin{array}{c}\text { Aloe vera }(\text { L.) Burm.f. } \\
\text { Syn. Aloe barbadensis } \\
\text { Mill./ } \\
\text { Asphodelaceae } \\
\text { Kowar gandal } \\
\text { SAN-SR-100 }\end{array}$ & $\begin{array}{l}\text { Herb / } \\
\text { W,C }\end{array}$ & Leaves/ Latex & $\begin{array}{c}\text { Skin problems, } \\
\text { febrifuge, malaria, } \\
\text { inflammation, obesity, } \\
\text { digestive problems }\end{array}$ & 6 & 0.12 & 50 & 0.14 & $\begin{array}{c}1^{\bullet}, 2^{*}, 3^{*}, 4^{*}, 5^{*}, 6^{*}, \\
7^{*}, 8^{*}, 9^{*}, 10^{*}, 11^{*}, \\
12^{*}, 13^{*}, 14^{*}, 15^{*} \\
16^{*}, 17^{\bullet}, 18^{*}, 19^{*} \\
20^{*}, 21^{*}, 22^{*}, 23^{*}\end{array}$ & \\
\hline 13 & $\begin{array}{c}\text { Alternanthera pungens } \\
\text { Kunth/ Amaranthaceae } \\
\text { Khaki booti } \\
\text { SAN-SR-68 }\end{array}$ & $\begin{array}{l}\text { Herb/ } \\
\text { W }\end{array}$ & $\begin{array}{l}\text { Leaves/ } \\
\text { Decoction }\end{array}$ & $\begin{array}{l}\text { Menstrual disorders, } \\
\text { febrifuge, body pain }\end{array}$ & 3 & 0.23 & 13 & 0.04 & $\begin{array}{c}1^{*}, 2^{*}, 3^{*}, 4^{*}, 5^{*}, 6^{*}, \\
7^{*}, 8^{\mathbf{4}}, 9^{*}, 10^{*}, 11^{*}, \\
12^{*}, 13^{*}, 14^{*}, 15^{*}, \\
16^{*}, 17^{*}, 18^{*}, 19^{*}, \\
2^{*}, 21^{*}, 22^{*}, 23^{*}\end{array}$ & \\
\hline 14 & $\begin{array}{c}\text { Amaranthus } \\
\text { graecizans L./ } \\
\text { Amaranthaceae } \\
\text { Marierri } \\
\text { SAN-SR-112 }\end{array}$ & $\begin{array}{l}\text { Herb/ } \\
\text { W }\end{array}$ & Leaves/ Cooked & $\begin{array}{c}\text { Laxative, } \\
\text { gastrointestinal } \\
\text { problems, galactagogue }\end{array}$ & 3 & 0.15 & 20 & 0.06 & $\begin{array}{c}1^{*}, 2^{*}, 3^{*}, 4^{*}, 5^{*}, 6^{*}, \\
7^{*}, 8^{*}, 9^{*}, 10^{*}, 11^{*}, \\
12^{*}, 13^{*}, 14^{*}, 15^{*}, \\
16^{*}, 17^{*}, 18^{*}, 19^{*}, \\
20^{\mathbf{4}}, 21^{*}, 22^{*}, 23^{*}\end{array}$ & \\
\hline 15 & $\begin{array}{c}\text { Amaranthus viridis L./ } \\
\text { Amaranthaceae } \\
\text { Bathu } \\
\text { SAN-SR-01 }\end{array}$ & $\begin{array}{c}\text { Herb/ } \\
\text { W }\end{array}$ & Leaves/ Cooked & $\begin{array}{c}\text { Laxative, } \\
\text { gastrointestinal } \\
\text { problems, respiratory } \\
\text { disorders }\end{array}$ & 3 & 0.15 & 20 & 0.06 & $\begin{array}{c}1^{*}, 2^{*}, 3^{\mathbf{4}}, 4^{*}, 5^{*}, 6^{*}, \\
7^{*}, 8^{\bullet}, 9^{*}, 10^{*}, 11^{*}, \\
12^{*}, 13^{*}, 14^{*}, 15^{*}, \\
16^{*}, 17^{*}, 18^{*}, 19^{*}, \\
20^{\mathbf{4}}, 21^{*}, 22^{*}, 23^{*}\end{array}$ & \\
\hline
\end{tabular}

APPLIED ECOLOGY AND ENVIRONMENTAL RESEARCH 17(2):4725-4805.

http://www.aloki.hu • ISSN 15891623 (Print) • ISSN 17850037 (Online)

DOI: http://dx.doi.org/10.15666/aeer/1702_47254805

(c) 2019, ALÖKI Kft., Budapest, Hungary 


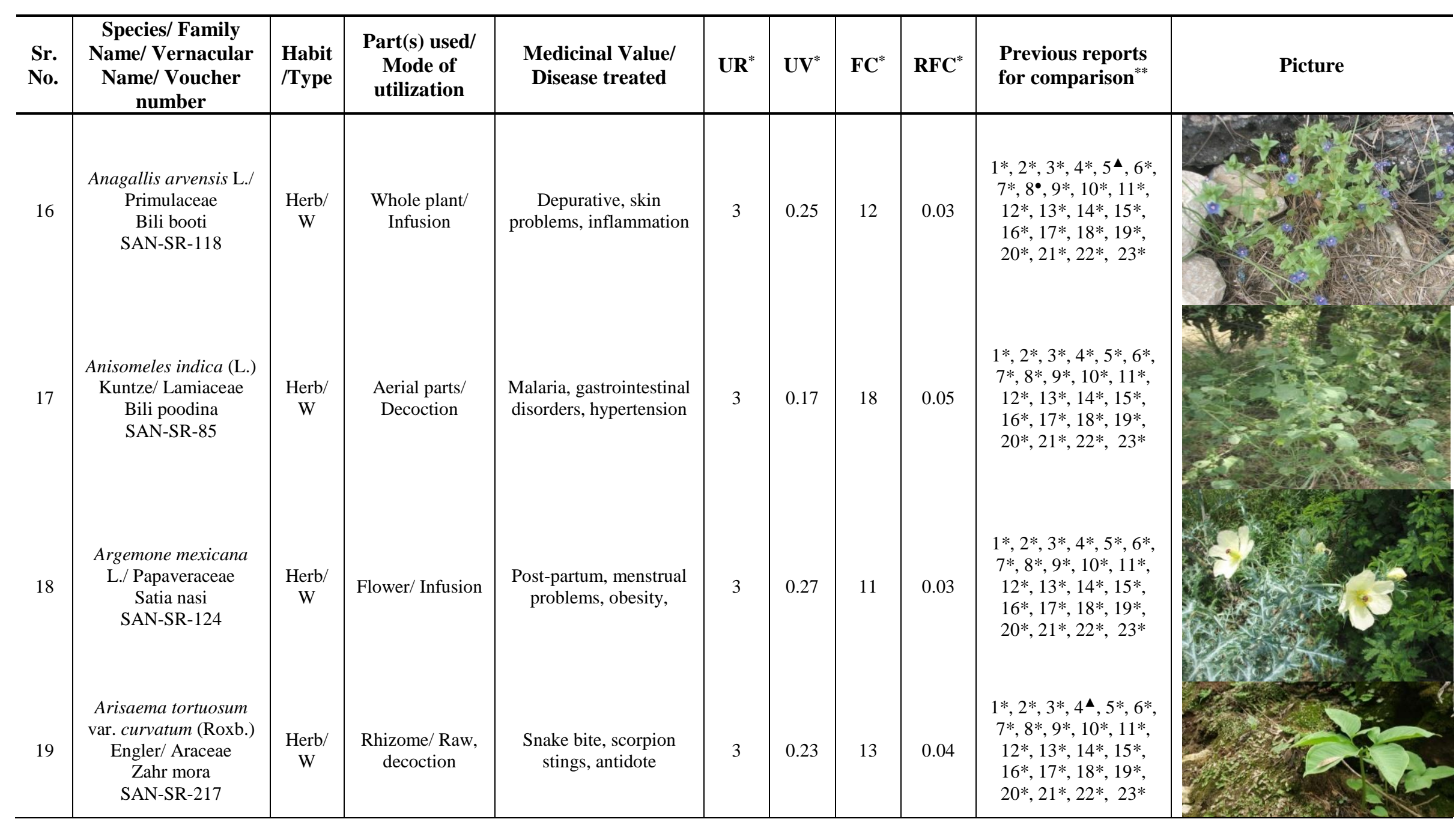

APPLIED ECOLOGY AND ENVIRONMENTAL RESEARCH 17(2):4725-4805.

http://www.aloki.hu • ISSN 15891623 (Print) • ISSN 17850037 (Online)

DOI: http://dx.doi.org/10.15666/aeer/1702_47254805

(c) 2019, ALÖKI Kft., Budapest, Hungary 


\begin{tabular}{|c|c|c|c|c|c|c|c|c|c|c|}
\hline $\begin{array}{l}\text { Sr. } \\
\text { No. }\end{array}$ & $\begin{array}{c}\text { Species/ Family } \\
\text { Name/ Vernacular } \\
\text { Name/ Voucher } \\
\text { number }\end{array}$ & $\begin{array}{l}\text { Habit } \\
\text { /Type }\end{array}$ & $\begin{array}{l}\text { Part(s) used/ } \\
\text { Mode of } \\
\text { utilization }\end{array}$ & $\begin{array}{l}\text { Medicinal Value/ } \\
\text { Disease treated }\end{array}$ & $\mathbf{U R}^{*}$ & $\mathbf{U V}^{*}$ & $\mathbf{F C}^{*}$ & RFC $^{*}$ & $\begin{array}{l}\text { Previous reports } \\
\text { for comparison }\end{array}$ & Picture \\
\hline 20 & $\begin{array}{c}\text { Arundo donax L./ } \\
\text { Poaceae } \\
\text { Narrki } \\
\text { SAN-SR-189 }\end{array}$ & $\begin{array}{l}\text { Herb/ } \\
\text { W }\end{array}$ & $\begin{array}{l}\text { Aerial parts/ } \\
\text { Decoction }\end{array}$ & $\begin{array}{l}\text { Fever, bloating, } \\
\text { menstrual problems }\end{array}$ & 3 & 0.42 & 7 & 0.02 & $\begin{array}{c}1^{*}, 2^{*}, 3^{\star}, 4^{*}, 5^{*}, 6^{*}, \\
7^{*}, 8^{*}, 9^{*}, 10^{*}, 11^{*}, \\
12^{*}, 13^{*}, 14^{*}, 15^{*} \\
16^{*}, 17^{*}, 18^{*}, 19^{*} \\
20^{*}, 21^{*}, 22^{*}, 23^{*}\end{array}$ & \\
\hline 21 & $\begin{array}{c}\text { Asparagus capitatus } \\
\text { Baker/ Asparagaceae } \\
\text { SAN-SR-210 }\end{array}$ & $\begin{array}{l}\text { Herb/ } \\
\text { W }\end{array}$ & $\begin{array}{l}\text { Whole plant/ } \\
\text { Decoction }\end{array}$ & $\begin{array}{l}\text { Body pain, fever, } \\
\text { gastrointestinal } \\
\text { problems }\end{array}$ & 3 & 0.16 & 19 & 0.05 & $\begin{array}{c}1^{*}, 2^{*}, 3^{*}, 4^{*}, 5^{*}, 6^{*}, \\
7^{*}, 8^{*}, 9^{*}, 10^{*}, 11^{*} \\
12^{*}, 13^{*}, 14^{*}, 15^{*} \\
16^{*}, 17^{*}, 18^{*}, 19^{*} \\
20^{*}, 21^{*}, 22^{*}, 23^{*}\end{array}$ & \\
\hline 22 & $\begin{array}{c}\text { Asphodelus tenuifolius } \\
\text { Cav./ Asphodelaceae } \\
\text { Wassli } \\
\text { SAN-SR-54 }\end{array}$ & $\begin{array}{c}\text { Herb/ } \\
\text { W }\end{array}$ & Seeds/ Raw & $\begin{array}{l}\text { Piles, febrifuge, } \\
\text { anthelmintic, ring worm }\end{array}$ & 4 & 0.18 & 22 & 0.06 & $\begin{array}{c}1^{*}, 2^{*}, 3^{*}, 4^{*}, 5^{*}, 6^{*} \\
7^{*}, 8^{*}, 9^{*}, 10^{*}, 11^{*} \\
12^{*}, 13^{*}, 14^{*}, 15^{*} \\
16^{*}, 17^{*}, 18^{*}, 19^{*} \\
20^{*}, 21^{*}, 22^{*}, 23^{*}\end{array}$ & \\
\hline
\end{tabular}




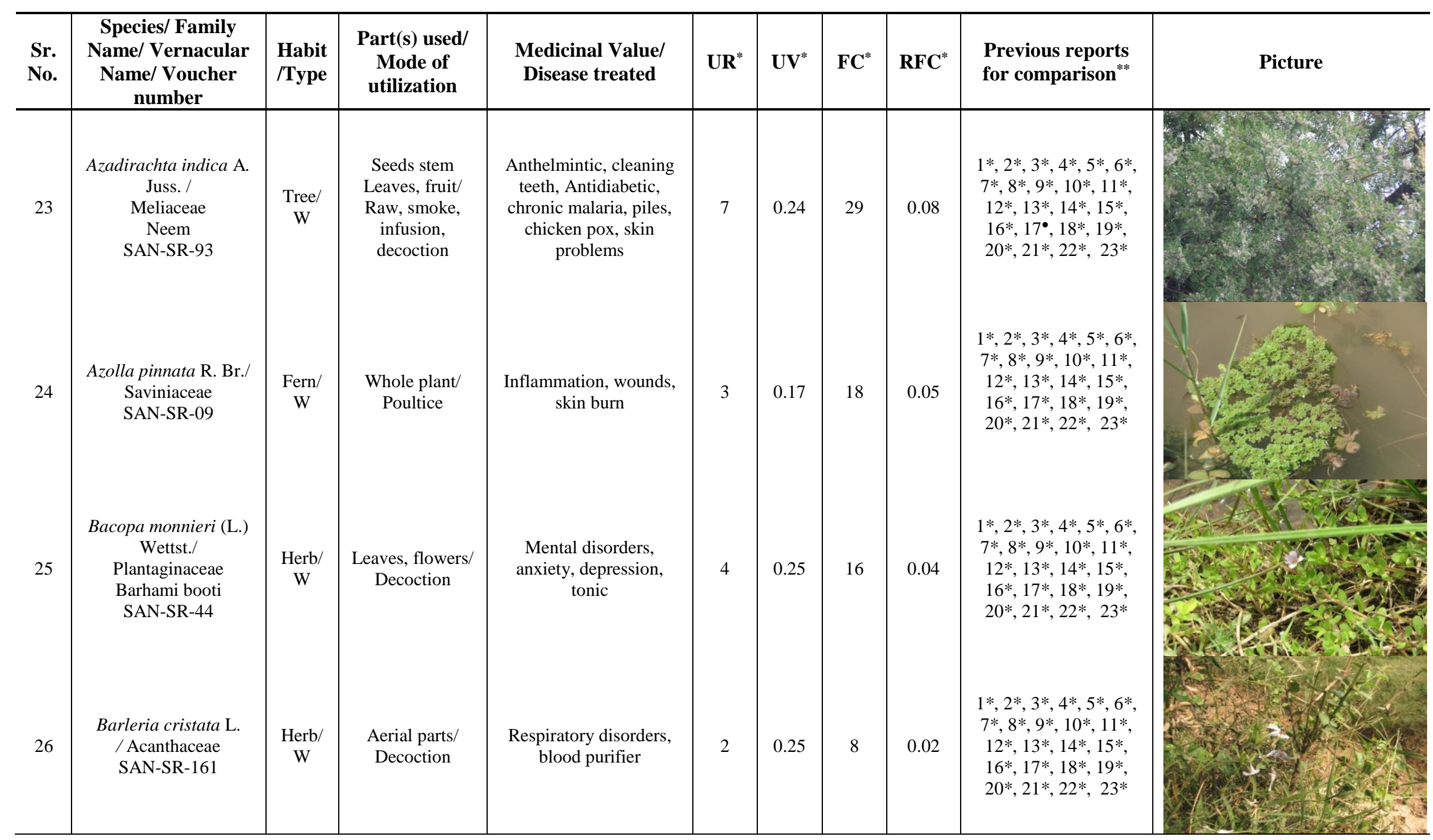




\begin{tabular}{|c|c|c|c|c|c|c|c|c|c|c|}
\hline $\begin{array}{l}\text { Sr. } \\
\text { No. }\end{array}$ & $\begin{array}{c}\text { Species/ Family } \\
\text { Name/ Vernacular } \\
\text { Name/ Voucher } \\
\text { number } \\
\end{array}$ & $\begin{array}{l}\text { Habit } \\
\text { /Type }\end{array}$ & $\begin{array}{l}\text { Part(s) used/ } \\
\text { Mode of } \\
\text { utilization }\end{array}$ & $\begin{array}{l}\text { Medicinal Value/ } \\
\text { Disease treated }\end{array}$ & $\mathbf{U} \mathbf{R}^{*}$ & $\mathbf{U V}^{*}$ & $\mathbf{F C}^{*}$ & RFC $^{*}$ & $\begin{array}{l}\text { Previous reports } \\
\text { for comparison** }\end{array}$ & Picture \\
\hline 27 & $\begin{array}{l}\text { Boerhavia procumbens } \\
\text { Banks ex. Roxb. } \\
\text { /Nyctaginaceae } \\
\text { It-sit } \\
\text { SAN-SR-61 }\end{array}$ & $\begin{array}{l}\text { Herb/ } \\
\text { W }\end{array}$ & $\begin{array}{l}\text { Leaves/ } \\
\text { Decoction }\end{array}$ & $\begin{array}{c}\text { Poor appetite, febrifuge, } \\
\text { snake bite, piles, } \\
\text { diuretic }\end{array}$ & 5 & 0.31 & 16 & 0.04 & $\begin{array}{c}1^{*}, 2^{*}, 3^{*}, 4^{*}, 5^{*}, 6^{*}, \\
7^{*}, 8^{*}, 9^{*}, 10^{*}, 11^{*}, \\
12^{*}, 13^{*}, 14^{*}, 15^{*}, \\
16^{*}, 17^{*}, 18^{*}, 19^{*}, \\
20^{*}, 21^{*}, 22^{*}, 23^{*}\end{array}$ & \\
\hline 28 & $\begin{array}{c}\text { Broussonetia } \\
\text { papyrifera (L.) Vent./ } \\
\text { Moraceae } \\
\text { Kagazi toot } \\
\text { SAN-SR-214 }\end{array}$ & $\begin{array}{l}\text { Tree/ } \\
\text { W }\end{array}$ & Leaves/ Infusion & Dysentery & 1 & 0.5 & 2 & 0.01 & $\begin{array}{c}1^{*}, 2^{*}, 3^{*}, 4^{*}, 5^{*}, 6^{*}, \\
7^{*}, 8^{*}, 9^{*}, 10^{*}, 11^{*}, \\
12^{*}, 13^{*}, 14^{*}, 15^{*}, \\
16^{*}, 17^{*}, 18^{*}, 19^{*}, \\
20^{*}, 21^{*}, 22^{*}, 23^{*}\end{array}$ & \\
\hline 29 & $\begin{array}{c}\text { Calligonum comosum } \\
\text { L'Hér./ Polygonaceae } \\
\text { Khippi } \\
\text { SAN-SR-200 }\end{array}$ & $\begin{array}{c}\text { Shrub/ } \\
\text { W }\end{array}$ & $\begin{array}{l}\text { Leaves/ } \\
\text { Decoction }\end{array}$ & $\begin{array}{l}\text { Digestive problems, } \\
\text { body pain, headache, }\end{array}$ & 3 & 0.17 & 17 & 0.05 & $\begin{array}{c}1^{*}, 2^{*}, 3^{*}, 4^{*}, 5^{*}, 6^{*}, \\
7^{*}, 8^{*}, 9^{*}, 10^{*}, 11^{*}, \\
12^{*}, 13^{*}, 14^{*}, 15^{*}, \\
16^{*}, 17^{*}, 18^{*}, 19^{*}, \\
20^{*}, 21^{*}, 22^{*}, 23^{*}\end{array}$ & \\
\hline 30 & $\begin{array}{l}\text { Calotropis procera } \\
\text { (Aiton) Dryand/ } \\
\text { Apocynaceae } \\
\text { Akrra } \\
\text { SAN-SR-16 }\end{array}$ & $\begin{array}{c}\text { Shrub/ } \\
\text { W }\end{array}$ & $\begin{array}{l}\text { Leaves, flower/ } \\
\text { Latex }\end{array}$ & $\begin{array}{l}\text { Snake bite, rheumatism, } \\
\text { wound healing, } \\
\text { febrifuge, mumps, } \\
\text { toothache }\end{array}$ & 6 & 0.13 & 45 & 0.13 & $\begin{array}{c}1^{*}, 2^{\star}, 3^{*}, 4^{*}, 5^{\bullet}, 6^{*}, \\
7^{*}, 8^{\bullet}, 9^{*}, 10^{*}, 11^{*}, \\
12^{*}, 13^{*}, 14^{*}, 15^{*}, \\
16^{*}, 17^{\bullet}, 18^{*}, 19^{*} \\
20^{*}, 21^{*}, 22^{*}, 23^{*}\end{array}$ & \\
\hline
\end{tabular}

APPLIED ECOLOGY AND ENVIRONMENTAL RESEARCH 17(2):4725-4805.

http://www.aloki.hu • ISSN 15891623 (Print) • ISSN 17850037 (Online)

DOI: http://dx.doi.org/10.15666/aeer/1702_47254805

(c) 2019, ALÖKI Kft., Budapest, Hungary 


\begin{tabular}{|c|c|c|c|c|c|c|c|c|c|c|}
\hline $\begin{array}{l}\text { Sr. } \\
\text { No. }\end{array}$ & $\begin{array}{c}\text { Species/ Family } \\
\text { Name/ Vernacular } \\
\text { Name/ Voucher } \\
\text { number } \\
\end{array}$ & $\begin{array}{l}\text { Habit } \\
\text { /Type }\end{array}$ & $\begin{array}{l}\text { Part(s) used/ } \\
\text { Mode of } \\
\text { utilization }\end{array}$ & $\begin{array}{l}\text { Medicinal Value/ } \\
\text { Disease treated }\end{array}$ & $\mathbf{U} \mathbf{R}^{*}$ & $\mathbf{U V} \mathbf{V}^{*}$ & $\mathbf{F C}^{*}$ & RFC $^{*}$ & $\begin{array}{l}\text { Previous reports } \\
\text { for comparison }\end{array}$ & Picture \\
\hline 31 & $\begin{array}{c}\text { Cannabis sativa } \text { L./ } \\
\text { Cannabaceae/ Bhang } \\
\text { SAN-SR-22 }\end{array}$ & $\begin{array}{l}\text { Herb/ } \\
\text { W }\end{array}$ & $\begin{array}{l}\text { Leaves/ Raw, } \\
\text { Decoction }\end{array}$ & $\begin{array}{l}\text { Insomnia, hypertension, } \\
\text { abdominal pain, } \\
\text { toothache }\end{array}$ & 4 & 0.16 & 25 & 0.07 & 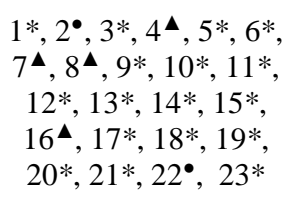 & \\
\hline 32 & $\begin{array}{c}\text { Capparis decidua } \\
\text { (Forssk.) Edgew./ } \\
\text { Capparaceae } \\
\text { Karein } \\
\text { SAN-SR-03 }\end{array}$ & $\begin{array}{c}\text { Tree/ } \\
\text { W }\end{array}$ & $\begin{array}{l}\text { Fruit, flower/ } \\
\text { Raw, juice }\end{array}$ & $\begin{array}{l}\text { Digestive problems, } \\
\text { tonic, obesity }\end{array}$ & 3 & 0.1 & 30 & 0.08 & $\begin{array}{c}1^{*}, 2^{*}, 3^{*}, 4^{*}, 5^{*}, 6^{*} \\
7^{*}, 8^{*}, 9^{*}, 10^{*}, 11^{*} \\
12^{*}, 13^{*}, 14^{*}, 15^{*} \\
16^{*}, 17^{\star}, 18^{*}, 19^{*} \\
20^{*}, 21^{*}, 22^{*}, 23^{*}\end{array}$ & \\
\hline 33 & $\begin{array}{l}\text { Capparis spinosa L./ } \\
\text { Capparaceae } \\
\text { SAN-SR-47 }\end{array}$ & $\begin{array}{l}\text { Shrub/ } \\
\text { W }\end{array}$ & $\begin{array}{l}\text { Stem, flower/ } \\
\text { Juice }\end{array}$ & Toothache, abortifacient & 2 & 0.25 & 8 & 0.02 & $\begin{array}{c}1^{*}, 2^{*}, 3^{*}, 4^{*}, 5^{*}, 6^{*}, \\
7^{*}, 8^{*}, 9^{*}, 10^{\mathbf{4}}, 11^{*}, \\
12^{*}, 13^{*}, 14^{*}, 15^{*} \\
16^{*}, 17^{*}, 18^{*}, 19^{*} \\
20^{*}, 21^{*}, 22^{*}, 23^{*}\end{array}$ & \\
\hline 34 & $\begin{array}{l}\text { Capsella bursa- } \\
\text { pastoris }(\mathrm{L} .) \text { Medik./ } \\
\text { Brassicaceae } \\
\text { SAN-SR-102 }\end{array}$ & $\begin{array}{l}\text { Herb/ } \\
\text { W }\end{array}$ & $\begin{array}{l}\text { Leaves/ Paste, } \\
\text { decoction }\end{array}$ & $\begin{array}{l}\text { Skin problems, } \\
\text { postpartum, cardiotonic }\end{array}$ & 3 & 0.16 & 19 & 0.05 & 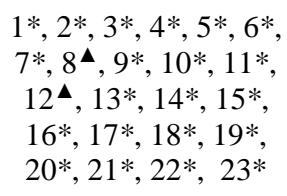 & \\
\hline
\end{tabular}

APPLIED ECOLOGY AND ENVIRONMENTAL RESEARCH 17(2):4725-4805.

http://www aloki.hu • ISSN 15891623 (Print) • ISSN 17850037 (Online)

DOI: http://dx.doi.org/10.15666/aeer/1702_47254805

(๖) 2019, ALÖKI Kft., Budapest, Hungary 


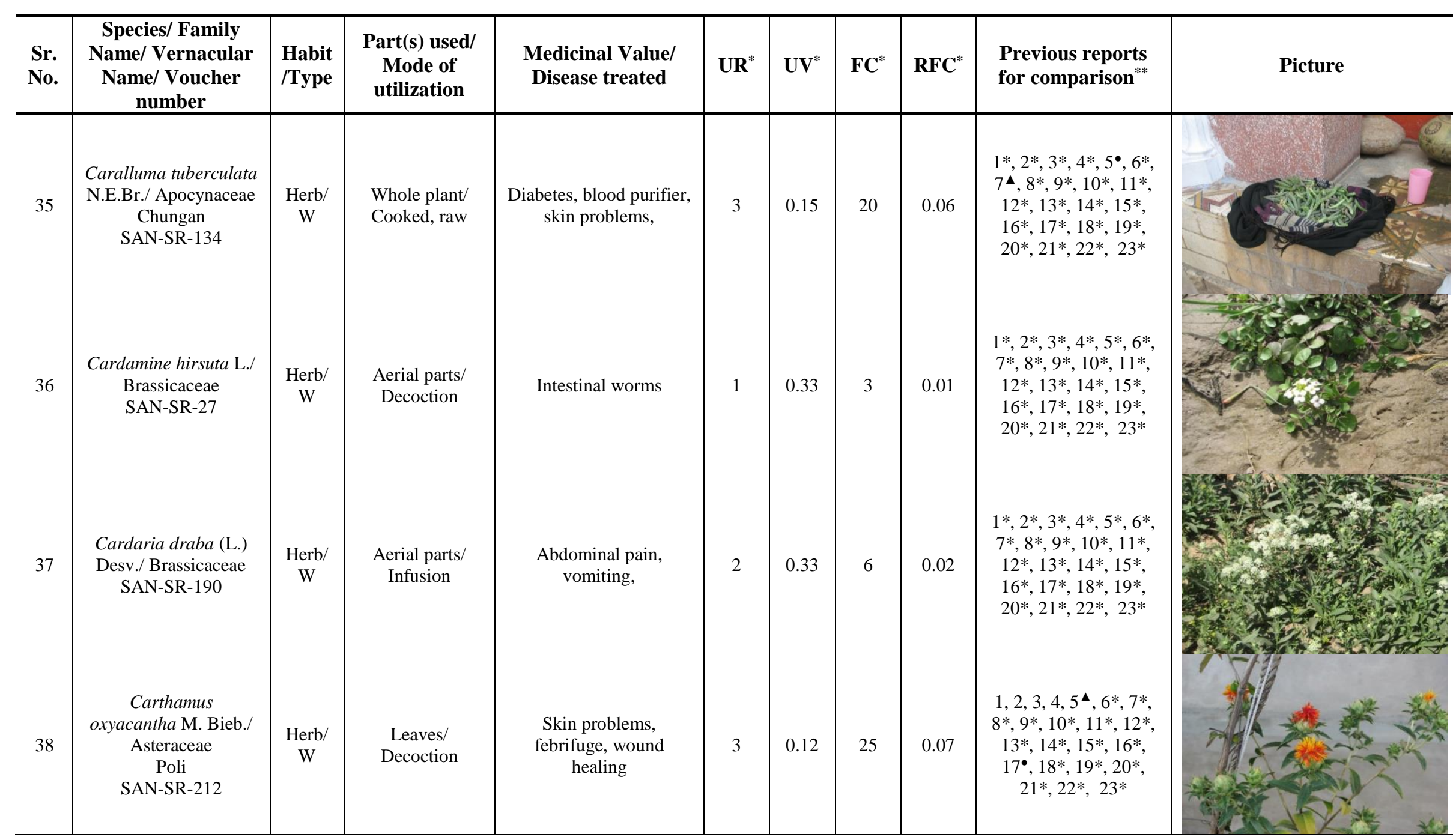

APPLIED ECOLOGY AND ENVIRONMENTAL RESEARCH 17(2):4725-4805.

http://www.aloki.hu • ISSN 15891623 (Print) • ISSN 17850037 (Online)

DOI: http://dx.doi.org/10.15666/aeer/1702_47254805

(c) 2019, ALÖKI Kft., Budapest, Hungary 


\begin{tabular}{|c|c|c|c|c|c|c|c|c|c|c|}
\hline $\begin{array}{l}\text { Sr. } \\
\text { No. }\end{array}$ & $\begin{array}{c}\text { Species/ Family } \\
\text { Name/ Vernacular } \\
\text { Name/ Voucher } \\
\text { number } \\
\end{array}$ & $\begin{array}{l}\text { Habit } \\
\text { /Type }\end{array}$ & $\begin{array}{l}\text { Part(s) used/ } \\
\text { Mode of } \\
\text { utilization }\end{array}$ & $\begin{array}{c}\text { Medicinal Value/ } \\
\text { Disease treated }\end{array}$ & $\mathbf{U R}^{*}$ & $\mathbf{U V}^{*}$ & $\mathbf{F C}^{*}$ & RFC $^{*}$ & $\begin{array}{l}\text { Previous reports } \\
\text { for comparison }\end{array}$ & Picture \\
\hline 39 & $\begin{array}{l}\text { Cenchrus echinatus } \\
\text { L./ Poaceae } \\
\text { SAN-SR-24 }\end{array}$ & $\begin{array}{l}\text { Herb/ } \\
\text { W }\end{array}$ & ----- & ----- & 0 & & 6 & 0.02 & $\begin{array}{c}1^{*}, 2^{*}, 3^{*}, 4^{*}, 5^{*}, 6^{*} \\
7^{*}, 8^{*}, 9^{*}, 10^{*}, 11^{*} \\
12^{*}, 13^{*}, 14^{*}, 15^{*} \\
16^{*}, 17^{*}, 18^{*}, 19^{*} \\
20^{*}, 21^{*}, 22^{*}, 23^{*}\end{array}$ & \\
\hline 40 & $\begin{array}{c}\text { Centaurium } \\
\text { pulchellum (Sw.) } \\
\text { Druce/ Gentianaceae } \\
\text { SAN-SR-66 }\end{array}$ & $\begin{array}{c}\text { Herb/ } \\
\text { W }\end{array}$ & $\begin{array}{l}\text { Leaves/ } \\
\text { Decoction }\end{array}$ & $\begin{array}{l}\text { Fever, kidney pain, } \\
\text { diuretic }\end{array}$ & 3 & 0.18 & 16 & 0.04 & $\begin{array}{c}1^{*}, 2^{*}, 3^{*}, 4^{*}, 5^{*}, 6^{*} \\
7^{*}, 8^{*}, 9^{*}, 10^{*}, 11^{*} \\
12^{*}, 13^{*}, 14^{*}, 15^{*} \\
16^{*}, 17^{*}, 18^{*}, 19^{*} \\
20^{*}, 21^{*}, 22^{*}, 23^{*}\end{array}$ & \\
\hline 41 & $\begin{array}{c}\text { Ceratonia siliqua } \text { L./ } \\
\text { Fabaceae } \\
\text { SAN-SR-125 }\end{array}$ & $\begin{array}{l}\text { Tree/ } \\
\text { W }\end{array}$ & $\begin{array}{c}\text { Seeds/ } \\
\text { Decoction }\end{array}$ & $\begin{array}{l}\text { Gastrointestinal } \\
\text { problems, obesity }\end{array}$ & 2 & 0.08 & 25 & 0.07 & $\begin{array}{c}1^{*}, 2^{*}, 3^{*}, 4^{*}, 5^{*}, 6^{*}, \\
7^{*}, 8^{*}, 9^{*}, 10^{*}, 11^{*} \\
12^{*}, 13^{*}, 14^{*}, 15^{*} \\
16^{*}, 17^{*}, 18^{*}, 19^{*} \\
20^{*}, 21^{*}, 22^{*}, 23^{*}\end{array}$ & \\
\hline 42 & $\begin{array}{c}\text { Chenopodium murale } \\
\text { L./ Chenopodiaceae } \\
\text { Dosaga } \\
\text { SAN-SR-43 }\end{array}$ & $\begin{array}{l}\text { Herb/ } \\
\text { W }\end{array}$ & Leaves/ Cooked & $\begin{array}{l}\text { Digestive problems, } \\
\text { menstrual problems }\end{array}$ & 2 & 0.12 & 17 & 0.05 & $\begin{array}{c}1^{*}, 2^{*}, 3^{*}, 4^{*}, 5^{*}, 6^{*}, \\
7^{*}, 8^{*}, 9^{*}, 10^{*}, 11^{*} \\
12^{*}, 13^{*}, 14^{*}, 15^{*} \\
16^{*}, 17^{*}, 18^{*}, 19^{*} \\
20^{*}, 21^{*}, 22^{*}, 23^{*}\end{array}$ & \\
\hline
\end{tabular}

APPLIED ECOLOGY AND ENVIRONMENTAL RESEARCH 17(2):4725-4805.

http://www.aloki.hu • ISSN 15891623 (Print) • ISSN 17850037 (Online)

DOI: http://dx.doi.org/10.15666/aeer/1702_47254805

(c) 2019, ALÖKI Kft., Budapest, Hungary 


\begin{tabular}{|c|c|c|c|c|c|c|c|c|c|c|}
\hline $\begin{array}{l}\text { Sr. } \\
\text { No. }\end{array}$ & $\begin{array}{c}\text { Species/ Family } \\
\text { Name/ Vernacular } \\
\text { Name/ Voucher } \\
\text { number } \\
\end{array}$ & $\begin{array}{l}\text { Habit } \\
\text { /Type }\end{array}$ & $\begin{array}{l}\text { Part(s) used/ } \\
\text { Mode of } \\
\text { utilization }\end{array}$ & $\begin{array}{l}\text { Medicinal Value/ } \\
\text { Disease treated }\end{array}$ & $\mathbf{U R}^{*}$ & $\mathbf{U V}^{*}$ & $\mathbf{F C}^{*}$ & $\mathbf{R F C}^{*}$ & $\begin{array}{l}\text { Previous reports } \\
\text { for comparison }\end{array}$ & Picture \\
\hline 43 & $\begin{array}{c}\text { Chenopodium album } \\
\text { L./ Chenopodiaceae } \\
\text { Chulaii } \\
\text { SAN-SR-122 }\end{array}$ & $\begin{array}{l}\text { Herb/ } \\
\text { W }\end{array}$ & Leaves/ Cooked & $\begin{array}{l}\text { Digestive problems, } \\
\text { menstrual problems }\end{array}$ & 2 & 0.12 & 17 & 0.05 & $\begin{array}{c}1^{*}, 2^{\mathbf{4}}, 3^{\mathbf{4}}, 4^{*}, 5^{\mathbf{4}}, \\
6^{\mathbf{4}}, 7^{\bullet}, 8^{\bullet}, 9^{*}, 10^{\mathbf{4}}, \\
11^{*}, 12^{\mathbf{4}}, 13^{*}, 14^{*} \\
15^{*}, 16^{*}, 17^{\mathbf{4}}, 18^{*}, \\
19^{*}, 20^{*}, 21^{*}, 22^{*}, \\
23^{*}\end{array}$ & \\
\hline 44 & $\begin{array}{c}\text { Chloris gayana Kunth/ } \\
\text { Poaceae } \\
\text { Pankha Ghaas } \\
\text { SAN-SR-148 }\end{array}$ & $\begin{array}{l}\text { Herb/ } \\
\text { W }\end{array}$ & ----- & ----- & 0 & & 12 & 0.03 & $\begin{array}{c}1,2,3,4,5,6,7,8,9 \\
10,11,12,13,14,15, \\
16,17,18,19,20,21 \\
22,23\end{array}$ & \\
\hline 45 & $\begin{array}{l}\text { Chrozophora tinctoria } \\
\text { (L.) A. Juss./ } \\
\text { Euphorbiaceae } \\
\text { Hathi sundi } \\
\text { SAN-SR-81 }\end{array}$ & $\begin{array}{l}\text { Herb/ } \\
\text { W }\end{array}$ & $\begin{array}{l}\text { Flowers/ } \\
\text { Infusion, } \\
\text { decoction }\end{array}$ & $\begin{array}{l}\text { Fever, stomach ache, } \\
\text { vomiting, wound } \\
\text { healing }\end{array}$ & 4 & 0.22 & 18 & 0.05 & $\begin{array}{c}1^{*}, 2^{*}, 3^{*}, 4^{*}, 5^{*}, 6^{*}, \\
7^{*}, 8^{*}, 9^{*}, 10^{*}, 11^{*}, \\
12^{*}, 13^{*}, 14^{*}, 15^{*}, \\
16^{*}, 17^{*}, 18^{*}, 19^{*}, \\
20^{*}, 21^{*}, 22^{*}, 23^{*}\end{array}$ & \\
\hline 46 & $\begin{array}{c}\text { Cichorium intybus L./ } \\
\text { Asteraceae } \\
\text { Kasni } \\
\text { SAN-SR-101 }\end{array}$ & $\begin{array}{l}\text { Herb/ } \\
\text { W }\end{array}$ & $\begin{array}{l}\text { Aerial parts/ } \\
\text { Decoction }\end{array}$ & $\begin{array}{l}\text { Kidney pain, gallbladder } \\
\text { stone, stomach ache }\end{array}$ & 3 & 0.25 & 12 & 0.03 & $\begin{array}{c}1^{*}, 2^{\bullet}, 3^{\star}, 4^{*}, 5^{*}, 6^{*}, \\
7^{\bullet}, 8^{\bullet}, 9^{*}, 10^{\bullet}, 11^{*}, \\
12^{*}, 13^{*}, 14^{*}, 15^{*}, \\
16^{*}, 17^{*}, 18^{*}, 19^{*}, \\
20^{*}, 21^{*}, 22^{*}, 23^{*}\end{array}$ & \\
\hline
\end{tabular}

APPLIED ECOLOGY AND ENVIRONMENTAL RESEARCH 17(2):4725-4805.

http://www.aloki.hu • ISSN 15891623 (Print) • ISSN 17850037 (Online)

DOI: http://dx.doi.org/10.15666/aeer/1702_47254805

(c) 2019, ALÖKI Kft., Budapest, Hungary 


\begin{tabular}{|c|c|c|c|c|c|c|c|c|c|c|}
\hline $\begin{array}{l}\text { Sr. } \\
\text { No. }\end{array}$ & $\begin{array}{c}\text { Species/ Family } \\
\text { Name/ Vernacular } \\
\text { Name/ Voucher } \\
\text { number } \\
\end{array}$ & $\begin{array}{l}\text { Habit } \\
\text { /Type }\end{array}$ & $\begin{array}{l}\text { Part(s) used/ } \\
\text { Mode of } \\
\text { utilization }\end{array}$ & $\begin{array}{c}\text { Medicinal Value/ } \\
\text { Disease treated }\end{array}$ & $\mathbf{U R}^{*}$ & $\mathbf{U V} \mathbf{V}^{*}$ & $\mathbf{F C}^{*}$ & RFC $^{*}$ & $\begin{array}{l}\text { Previous reports } \\
\text { for comparison }\end{array}$ & Picture \\
\hline 47 & $\begin{array}{c}\text { Cirsium vulgare (Savi) } \\
\text { Ten./ Asteraceae } \\
\text { Laih } \\
\text { SAN-SR-133 }\end{array}$ & $\begin{array}{l}\text { Herb/ } \\
\text { W }\end{array}$ & Seeds/ Raw & Tonic, obesity & 2 & 0.28 & 7 & 0.02 & $\begin{array}{c}1^{*}, 2^{*}, 3^{*}, 4^{*}, 5^{*}, 6^{*} \\
7^{*}, 8^{*}, 9^{*}, 10^{*}, 11^{*} \\
12^{*}, 13^{*}, 14^{*}, 15^{*} \\
16^{*}, 17^{*}, 18^{*}, 19^{*} \\
20^{*}, 21^{*}, 22^{*}, 23^{*}\end{array}$ & \\
\hline 48 & $\begin{array}{c}\text { Cistanche tubulosa } \\
\text { (Schenk) Wight/ } \\
\text { Orobanchaceae } \\
\text { Khar ghainrr } \\
\text { SAN-SR-56 }\end{array}$ & $\begin{array}{l}\text { Herb/ } \\
\text { W }\end{array}$ & $\begin{array}{l}\text { Whole plant/ } \\
\text { Decoction }\end{array}$ & Aphrodisiac & 1 & 0.2 & 5 & 0.01 & $\begin{array}{c}1^{*}, 2^{*}, 3^{*}, 4^{*}, 5^{*}, 6^{*} \\
7^{*}, 8^{*}, 9^{*}, 10^{*}, 11^{*} \\
12^{*}, 13^{*}, 14^{*}, 15^{*} \\
16^{*}, 17^{*}, 18^{*}, 19^{*} \\
20^{*}, 21^{*}, 22^{*}, 23^{*}\end{array}$ & \\
\hline 49 & $\begin{array}{l}\text { Citrullus colocynthis } \\
\text { (L.) Schrad./ } \\
\text { Cucurbitaceae } \\
\text { Tuma } \\
\text { SAN-SR-147 }\end{array}$ & $\begin{array}{c}\text { Herb/ } \\
\text { W }\end{array}$ & Seeds/ Raw & $\begin{array}{l}\text { Diabetes, malaria, } \\
\text { stomach problems }\end{array}$ & 3 & 0.25 & 12 & 0.03 & $\begin{array}{c}1^{*}, 2^{*}, 3^{*}, 4^{*}, 5^{*}, 6^{*} \\
7^{*}, 8^{*}, 9^{*}, 10^{*}, 11^{*} \\
12^{*}, 13^{*}, 14^{*}, 15^{*} \\
16^{*}, 17^{*}, 18^{*}, 19^{*} \\
20^{*}, 21^{*}, 22^{*}, 23^{*}\end{array}$ & \\
\hline
\end{tabular}

APPLIED ECOLOGY AND ENVIRONMENTAL RESEARCH 17(2):4725-4805.

http://www.aloki.hu • ISSN 15891623 (Print) • ISSN 17850037 (Online)

DOI: http://dx.doi.org/10.15666/aeer/1702_47254805

(๖) 2019, ALÖKI Kft., Budapest, Hungary 


\begin{tabular}{|c|c|c|c|c|c|c|c|c|c|c|}
\hline $\begin{array}{l}\text { Sr. } \\
\text { No. }\end{array}$ & $\begin{array}{c}\text { Species/ Family } \\
\text { Name/ Vernacular } \\
\text { Name/ Voucher } \\
\text { number }\end{array}$ & $\begin{array}{l}\text { Habit } \\
\text { /Type }\end{array}$ & $\begin{array}{l}\text { Part(s) used/ } \\
\text { Mode of } \\
\text { utilization }\end{array}$ & $\begin{array}{l}\text { Medicinal Value/ } \\
\text { Disease treated }\end{array}$ & $\mathbf{U} \mathbf{R}^{*}$ & $\mathbf{U V}^{*}$ & FC $^{*}$ & RFC $^{*}$ & $\begin{array}{l}\text { Previous reports } \\
\text { for comparison }{ }^{* *}\end{array}$ & Picture \\
\hline 50 & $\begin{array}{c}\text { Clematis grata Wall./ } \\
\text { Ranunculaceae } \\
\text { SAN-SR-203 }\end{array}$ & $\begin{array}{l}\text { Shrub/ } \\
\text { W }\end{array}$ & $\begin{array}{l}\text { Leaves/ Paste, } \\
\text { infusion }\end{array}$ & $\begin{array}{l}\text { Skin problems, } \\
\text { vomiting, body pain }\end{array}$ & 3 & 0.33 & 9 & 0.02 & $\begin{array}{c}1^{*}, 2^{\mathbf{4}}, 3^{*}, 4^{*}, 5^{*}, 6^{*} \\
7^{*}, 8^{\mathbf{4}}, 9^{*}, 10^{*}, 11^{*} \\
12^{*}, 13^{*}, 14^{*}, 15^{*} \\
16^{*}, 17^{*}, 18^{*}, 19^{*} \\
20^{*}, 21^{*}, 22^{*}, 23^{*}\end{array}$ & \\
\hline 51 & $\begin{array}{l}\text { Clerodendrum } \\
\text { phlomidis L.f./ } \\
\text { Lamiaceae } \\
\text { SAN-SR-211 }\end{array}$ & $\begin{array}{c}\text { Shrub/ } \\
\text { W }\end{array}$ & $\begin{array}{l}\text { Leaves/ } \\
\text { Decoction }\end{array}$ & $\begin{array}{l}\text { Sore throat, respiratory } \\
\text { problems }\end{array}$ & 2 & 0.5 & 4 & 0.01 & $\begin{array}{c}1^{*}, 2^{*}, 3^{*}, 4^{*}, 5^{*}, 6^{*} \\
7^{*}, 8^{*}, 9^{*}, 10^{*}, 11^{*} \\
12^{*}, 13^{*}, 14^{*}, 15^{*} \\
16^{*}, 17^{*}, 18^{*}, 19^{*} \\
20^{*}, 21^{*}, 22^{*}, 23^{*}\end{array}$ & \\
\hline 52 & $\begin{array}{c}\text { Cocculus hirsutus (L.) } \\
\text { Diels/ } \\
\text { Menispermaceae } \\
\text { SAN-SR-174 }\end{array}$ & $\begin{array}{c}\text { Shrub/ } \\
\text { W }\end{array}$ & Fruit/ Decoction & $\begin{array}{l}\text { Malaria, body pain, } \\
\text { obesity }\end{array}$ & 3 & 0.43 & 7 & 0.02 & 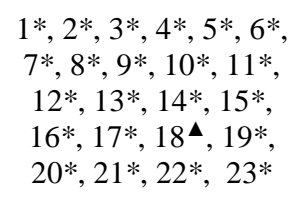 & \\
\hline 53 & $\begin{array}{c}\text { Cocculus pendulus } \\
\text { (J.R.Forst. \& G.Forst.) } \\
\text { Diels/ } \\
\text { Menispermaceae } \\
\text { SAN-SR-215 }\end{array}$ & $\begin{array}{l}\text { Shrub/ } \\
\text { W }\end{array}$ & Fruit/ Decoction & $\begin{array}{l}\text { Malaria, body pain, } \\
\text { obesity }\end{array}$ & 3 & 0.37 & 8 & 0.02 & $\begin{array}{c}1^{*}, 2^{*}, 3^{*}, 4^{*}, 5^{*}, 6^{*} \\
7^{*}, 8^{*}, 9^{*}, 10^{*}, 11^{*} \\
12^{*}, 13^{*}, 14^{*}, 15^{*} \\
16^{*}, 17^{*}, 18^{*}, 19^{*} \\
20^{*}, 21^{*}, 22^{*}, 23^{*}\end{array}$ & \\
\hline
\end{tabular}

APPLIED ECOLOGY AND ENVIRONMENTAL RESEARCH 17(2):4725-4805.

http://www.aloki.hu • ISSN 15891623 (Print) • ISSN 17850037 (Online)

DOI: http://dx.doi.org/10.15666/aeer/1702_47254805

(c) 2019, ALÖKI Kft., Budapest, Hungary 


\begin{tabular}{|c|c|c|c|c|c|c|c|c|c|c|}
\hline $\begin{array}{l}\text { Sr. } \\
\text { No. }\end{array}$ & $\begin{array}{c}\text { Species/ Family } \\
\text { Name/ Vernacular } \\
\text { Name/ Voucher } \\
\text { number } \\
\end{array}$ & $\begin{array}{l}\text { Habit } \\
\text { /Type }\end{array}$ & $\begin{array}{l}\text { Part(s) used/ } \\
\text { Mode of } \\
\text { utilization }\end{array}$ & $\begin{array}{l}\text { Medicinal Value/ } \\
\text { Disease treated }\end{array}$ & $\mathbf{U R}^{*}$ & $\mathbf{U V}^{*}$ & $\mathbf{F C}^{*}$ & $\mathbf{R F C}^{*}$ & $\begin{array}{l}\text { Previous reports } \\
\text { for comparison }\end{array}$ & Picture \\
\hline 54 & $\begin{array}{c}\text { Coix lacryma-jobi L./ } \\
\text { Poaceae } \\
\text { SAN-SR-79 }\end{array}$ & $\begin{array}{l}\text { Herb/ } \\
\text { W }\end{array}$ & ----- & ----- & 0 & 0 & 10 & 0.02 & $\begin{array}{c}1,2,3,4,5,6,7,8,9 \\
10,11,12,13,14,15 \\
16,17,18,19,20,21 \\
22,23\end{array}$ & \\
\hline 55 & $\begin{array}{c}\text { Convolvulus arvensis } \\
\text { L./ Convolvulaceae } \\
\text { Verri } \\
\text { SAN-SR-10 }\end{array}$ & $\begin{array}{l}\text { Herb/ } \\
\text { W }\end{array}$ & $\begin{array}{l}\text { Whole plant/ } \\
\text { Decoction }\end{array}$ & $\begin{array}{l}\text { Wound healing, } \\
\text { stomach problems }\end{array}$ & 2 & 0.12 & 16 & 0.04 & 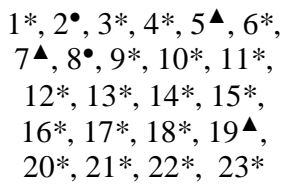 & \\
\hline 56 & $\begin{array}{c}\text { Convolvulus } \\
\text { prostratus Forssk./ } \\
\text { Convolvulaceae } \\
\text { SAN-SR-13 }\end{array}$ & $\begin{array}{l}\text { Herb/ } \\
\text { W }\end{array}$ & $\begin{array}{l}\text { Whole plant/ } \\
\text { Decoction }\end{array}$ & $\begin{array}{l}\text { Wound healing, } \\
\text { stomach problems }\end{array}$ & 2 & 0.12 & 16 & 0.04 & $\begin{array}{c}1^{*}, 2^{*}, 3^{*}, 4^{*}, 5^{*}, 6^{*} \\
7^{*}, 8^{*}, 9^{*}, 10^{*}, 11^{*} \\
12^{*}, 13^{*}, 14^{*}, 15^{*} \\
16^{*}, 17^{*}, 18^{*}, 19^{*} \\
20^{*}, 21^{*}, 22^{*}, 23^{*}\end{array}$ & \\
\hline 57 & $\begin{array}{l}\text { Conyza canadensis } \\
\text { (L.) Cronquist./ } \\
\text { Asteraceae } \\
\text { Paleet } \\
\text { SAN-SR-126 }\end{array}$ & $\begin{array}{l}\text { Herb/ } \\
\text { W }\end{array}$ & $\begin{array}{l}\text { Aerial parts/ } \\
\text { Decoction }\end{array}$ & Dysentery, diarrhea & 2 & 0.06 & 32 & 0.09 & $\begin{array}{c}1^{*}, 2^{*}, 3^{*}, 4^{*}, 5^{*}, 6^{*} \\
7^{*}, 8^{\mathbf{4}}, 9^{*}, 10^{*}, 11^{*} \\
12^{*}, 13^{*}, 14^{*}, 15^{*} \\
16^{*}, 17^{*}, 18^{*}, 19^{*} \\
20^{*}, 21^{*}, 22^{*}, 23^{*}\end{array}$ & \\
\hline
\end{tabular}

APPLIED ECOLOGY AND ENVIRONMENTAL RESEARCH 17(2):4725-4805.

http://www.aloki.hu • ISSN 15891623 (Print) • ISSN 17850037 (Online)

DOI: http://dx.doi.org/10.15666/aeer/1702_47254805

(c) 2019, ALÖKI Kft., Budapest, Hungary 


\begin{tabular}{|c|c|c|c|c|c|c|c|c|c|c|}
\hline $\begin{array}{l}\text { Sr. } \\
\text { No. }\end{array}$ & $\begin{array}{c}\text { Species/ Family } \\
\text { Name/ Vernacular } \\
\text { Name/ Voucher } \\
\text { number } \\
\end{array}$ & $\begin{array}{l}\text { Habit } \\
\text { /Type }\end{array}$ & $\begin{array}{l}\text { Part(s) used/ } \\
\text { Mode of } \\
\text { utilization }\end{array}$ & $\begin{array}{l}\text { Medicinal Value/ } \\
\text { Disease treated }\end{array}$ & $\mathbf{U R}^{*}$ & $\mathbf{U V}^{*}$ & $\mathbf{F C}^{*}$ & $\mathbf{R F C}^{*}$ & $\begin{array}{l}\text { Previous reports } \\
\text { for comparison }{ }^{* *}\end{array}$ & Picture \\
\hline 58 & $\begin{array}{c}\text { Croton } \\
\text { bonplandianum Baill./ } \\
\text { Euphorbiaceae } \\
\text { SAN-SR-25 }\end{array}$ & $\begin{array}{c}\text { Herb/ } \\
\text { W }\end{array}$ & $\begin{array}{l}\text { Leaf, seeds/ } \\
\text { Infusion, raw }\end{array}$ & $\begin{array}{l}\text { Blood purifier, } \\
\text { cardiotonic, constipation }\end{array}$ & 3 & 0.2 & 15 & 0.04 & $\begin{array}{c}1^{*}, 2^{*}, 3^{*}, 4^{*}, 5^{*}, 6^{*} \\
7^{*}, 8^{*}, 9^{*}, 10^{*}, 11^{*} \\
12^{*}, 13^{*}, 14^{*}, 15^{*} \\
16^{*}, 17^{*}, 18^{*}, 19^{*} \\
20^{*}, 21^{*}, 22^{*}, 23^{*}\end{array}$ & \\
\hline 59 & $\begin{array}{c}\text { Cucumis melo var. } \\
\text { agrestis Naudin/ } \\
\text { Cucurbitaceae } \\
\text { Chibbarr } \\
\text { SAN-SR-80 }\end{array}$ & $\begin{array}{l}\text { Herb/ } \\
\text { W }\end{array}$ & $\begin{array}{l}\text { Fruit, seeds/ } \\
\text { Raw, }\end{array}$ & $\begin{array}{l}\text { Purgative, cooling } \\
\text { effect, bloating, tonic }\end{array}$ & 4 & 0.21 & 19 & 0.05 & $\begin{array}{c}1^{*}, 2^{*}, 3^{*}, 4^{*}, 5^{*}, 6^{*} \\
7^{*}, 8^{*}, 9^{*}, 10^{*}, 11^{*} \\
12^{*}, 13^{*}, 14^{*}, 15^{*} \\
16^{*}, 17^{*}, 18^{*}, 19^{*} \\
20^{*}, 21^{*}, 22^{*}, 23^{*}\end{array}$ & \\
\hline 60 & $\begin{array}{c}\text { Cuscuta reflexa } \text { Roxb./ } \\
\text { Convolvulaceae } \\
\text { Akash-bail } \\
\text { SAN-SR-07 }\end{array}$ & $\begin{array}{c}\text { Herb/ } \\
\text { W }\end{array}$ & $\begin{array}{l}\text { Whole plant/ } \\
\text { Decoction }\end{array}$ & $\begin{array}{l}\text { Gastrointestinal } \\
\text { problems }\end{array}$ & 1 & 0.5 & 2 & 0.01 & $\begin{array}{c}1^{*}, 2^{*}, 3^{*}, 4^{\mathbf{4}}, 5^{*}, 6^{*}, \\
7^{*}, 8^{\mathbf{4}}, 9^{*}, 10^{*}, 11^{*}, \\
12^{*}, 13^{*}, 14^{*}, 15^{*} \\
16^{*}, 17^{*}, 18^{*}, 19^{*} \\
20^{*}, 21^{*}, 22^{*}, 23^{*}\end{array}$ & \\
\hline
\end{tabular}




\begin{tabular}{|c|c|c|c|c|c|c|c|c|c|c|}
\hline $\begin{array}{l}\text { Sr. } \\
\text { No. }\end{array}$ & $\begin{array}{c}\text { Species/ Family } \\
\text { Name/ Vernacular } \\
\text { Name/ Voucher } \\
\text { number } \\
\end{array}$ & $\begin{array}{l}\text { Habit } \\
\text { /Type }\end{array}$ & $\begin{array}{l}\text { Part(s) used/ } \\
\text { Mode of } \\
\text { utilization }\end{array}$ & $\begin{array}{l}\text { Medicinal Value/ } \\
\text { Disease treated }\end{array}$ & $\mathbf{U R}^{*}$ & $\mathbf{U V}^{*}$ & $\mathbf{F C}^{*}$ & RFC $^{*}$ & $\begin{array}{l}\text { Previous reports } \\
\text { for comparison }^{* *}\end{array}$ & Picture \\
\hline 61 & $\begin{array}{c}\text { Cymbopogon } \\
\text { commutatus (Steud.) } \\
\text { Stapf/ Poaceae } \\
\text { Jangli lemon grass } \\
\text { SAN-SR-157 }\end{array}$ & $\begin{array}{l}\text { Herb/ } \\
\text { W }\end{array}$ & $\begin{array}{l}\text { Aerial parts/ } \\
\text { Decoction }\end{array}$ & $\begin{array}{l}\text { Respiratory disorders, } \\
\text { obesity, digestive } \\
\text { problems, menstrual } \\
\text { pain }\end{array}$ & 4 & 0.17 & 24 & 0.07 & $\begin{array}{c}1^{*}, 2^{*}, 3^{*}, 4^{*}, 5^{*}, 6^{*}, \\
7^{*}, 8^{*}, 9^{*}, 10^{*}, 11^{*}, \\
12^{*}, 13^{*}, 14^{*}, 15^{*}, \\
16^{*}, 17^{*}, 18^{*}, 19^{*}, \\
20^{*}, 21^{*}, 22^{*}, 23^{*}\end{array}$ & \\
\hline 62 & $\begin{array}{c}\text { Cynodon dactylon (L.) } \\
\text { Pers./ Poaceae } \\
\text { Tulla } \\
\text { SAN-SR-166 }\end{array}$ & $\begin{array}{l}\text { Herb/ } \\
\text { W }\end{array}$ & $\begin{array}{l}\text { Leaves/ } \\
\text { Decoction }\end{array}$ & $\begin{array}{l}\text { Dysmenorrhea, } \\
\text { vomiting, piles, }\end{array}$ & 3 & 0.15 & 20 & 0.06 & $\begin{array}{c}1^{\bullet}, 2^{\bullet}, 3^{\mathbf{4}}, 4^{\mathbf{4}}, 5^{*}, 6^{*}, \\
7^{*}, 8^{\mathbf{4}}, 9^{*}, 10^{*}, 11^{*}, \\
12^{*}, 13^{*}, 14^{*}, 15^{\mathbf{4}}, \\
16^{\bullet}, 17^{*}, 18^{*}, 19^{*} \\
20^{*}, 21^{*}, 22^{*}, 23^{*}\end{array}$ & \\
\hline 63 & $\begin{array}{c}\text { Dactyloctenium } \\
\text { aegyptium }(\text { L.) Willd. / } \\
\text { Poaceae } \\
\text { Pankha Khabbal } \\
\text { SAN-SR-153 }\end{array}$ & $\begin{array}{c}\text { Herb/ } \\
\text { W }\end{array}$ & ---- & ----- & 0 & 0 & 17 & 0.05 & $\begin{array}{c}1,2,3,4,5,6,7,8,9, \\
10,11,12,13,14,15, \\
16,17,18,19,20,21, \\
22,23\end{array}$ & \\
\hline
\end{tabular}




\begin{tabular}{|c|c|c|c|c|c|c|c|c|c|c|}
\hline $\begin{array}{l}\text { Sr. } \\
\text { No. }\end{array}$ & $\begin{array}{c}\text { Species/ Family } \\
\text { Name/ Vernacular } \\
\text { Name/ Voucher } \\
\text { number } \\
\end{array}$ & $\begin{array}{l}\text { Habit } \\
\text { /Type }\end{array}$ & $\begin{array}{l}\text { Part(s) used/ } \\
\text { Mode of } \\
\text { utilization }\end{array}$ & $\begin{array}{l}\text { Medicinal Value/ } \\
\text { Disease treated }\end{array}$ & $\mathbf{U} \mathbf{R}^{*}$ & $\mathbf{U V} \mathbf{V}^{*}$ & $\mathbf{F C}^{*}$ & RFC $^{*}$ & $\begin{array}{l}\text { Previous reports } \\
\text { for comparison }\end{array}$ & Picture \\
\hline 64 & $\begin{array}{c}\text { Dalbergia sissoo DC./ } \\
\text { Fabaceae } \\
\text { Shisham, Tahli } \\
\text { SAN-SR-23 }\end{array}$ & $\begin{array}{l}\text { Tree/ } \\
\text { W,C }\end{array}$ & $\begin{array}{l}\text { Leaves, stem/ } \\
\text { Juice, bark }\end{array}$ & $\begin{array}{c}\text { Eye ailments, scabies, } \\
\text { dysmenorrhea }\end{array}$ & 3 & 0.10 & 29 & 0.08 & $\begin{array}{c}1^{*}, 2^{*}, 3^{\mathbf{4}}, 4^{*}, 5^{\mathbf{\Delta}}, 6^{*} \\
7^{*}, 8^{\mathbf{4}}, 9^{*}, 10^{*}, 11^{*} \\
12^{*}, 13^{*}, 14^{*}, 15^{*} \\
16^{*}, 17^{*}, 18^{\mathbf{4}}, 19^{*} \\
20^{*}, 21^{*}, 22^{*}, 23^{*}\end{array}$ & \\
\hline 65 & $\begin{array}{l}\text { Datura metel L./ } \\
\text { Solanaceae } \\
\text { Dhatura } \\
\text { SAN-SR-106 }\end{array}$ & $\begin{array}{l}\text { Herb/ } \\
\text { W }\end{array}$ & Seeds/ Powder & $\begin{array}{l}\text { Body pain, wound } \\
\text { healing }\end{array}$ & 2 & 0.08 & 25 & 0.07 & $\begin{array}{c}1^{*}, 2^{*}, 3^{*}, 4^{*}, 5^{*}, 6^{*} \\
7^{*}, 8^{*}, 9^{*}, 10^{*}, 11^{*} \\
12^{*}, 13^{*}, 14^{*}, 15^{*} \\
16^{*}, 17^{\star}, 18^{*}, 19^{*} \\
20^{*}, 21^{*}, 22^{*}, 23^{*}\end{array}$ & \\
\hline 66 & $\begin{array}{c}\text { Desmostachya } \\
\text { bipinnata }(\mathrm{L}) \text { Stapf./ } \\
\text { Poaceae } \\
\text { Dabb Ghaas } \\
\text { SAN-SR-168 }\end{array}$ & $\begin{array}{l}\text { Herb/ } \\
\text { W }\end{array}$ & ----- & ----- & 0 & 0 & 22 & 0.06 & $\begin{array}{c}1,2,3,4,5,6,7,8,9 \\
10,11,12,13,14,15 \\
16,17,18,19,20,21, \\
22,23\end{array}$ & \\
\hline
\end{tabular}




\begin{tabular}{|c|c|c|c|c|c|c|c|c|c|c|}
\hline $\begin{array}{l}\text { Sr. } \\
\text { No. }\end{array}$ & $\begin{array}{c}\text { Species/ Family } \\
\text { Name/ Vernacular } \\
\text { Name/ Voucher } \\
\text { number } \\
\end{array}$ & $\begin{array}{l}\text { Habit } \\
\text { /Type }\end{array}$ & $\begin{array}{l}\text { Part(s) used/ } \\
\text { Mode of } \\
\text { utilization }\end{array}$ & $\begin{array}{l}\text { Medicinal Value/ } \\
\text { Disease treated }\end{array}$ & $\mathbf{U R}^{*}$ & $\mathbf{U V}^{*}$ & $\mathbf{F C}^{*}$ & RFC $^{*}$ & $\begin{array}{l}\text { Previous reports } \\
\text { for comparison }\end{array}$ & Picture \\
\hline 67 & $\begin{array}{c}\text { Dichanthium } \\
\text { annulatum (Forssk.) } \\
\text { Stapf/ Poaceae } \\
\text { SAN-SR-196 }\end{array}$ & $\begin{array}{c}\text { Herb/ } \\
\text { W }\end{array}$ & ----- & ----- & 0 & 0 & 4 & 0.01 & $\begin{array}{l}1,2,3,4,5,6,7,8,9, \\
10,11,12,13,14,15, \\
16,17,18,19,20,21, \\
22,23\end{array}$ & \\
\hline 68 & $\begin{array}{c}\text { Dicliptera } \\
\text { bupleuroides Nees/ } \\
\text { Acanthaceae } \\
\text { SAN-SR-213 }\end{array}$ & $\begin{array}{l}\text { Herb/ } \\
\text { W }\end{array}$ & $\begin{array}{l}\text { Leaves/ } \\
\text { Decoction }\end{array}$ & $\begin{array}{l}\text { Stomach problems, } \\
\text { constipation }\end{array}$ & 2 & 0.33 & 6 & 0.02 & 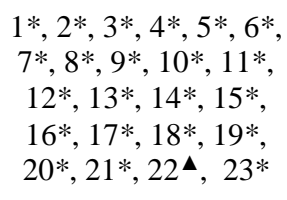 & \\
\hline 69 & $\begin{array}{c}\text { Digera muricata } \\
\text { (L.)Mart./ } \\
\text { Amaranthaceae } \\
\text { Tandla } \\
\text { SAN-SR-198 }\end{array}$ & $\begin{array}{c}\text { Herb/ } \\
\text { W }\end{array}$ & $\begin{array}{l}\text { Whole plant/ } \\
\text { Cooked, juice }\end{array}$ & $\begin{array}{l}\text { Urinary problems, } \\
\text { constipation, } \\
\text { inflammation }\end{array}$ & 3 & 0.21 & 14 & 0.04 & $\begin{array}{c}1^{*}, 2^{*}, 3^{*}, 4^{*}, 5^{*}, 6^{*}, \\
7^{*}, 8^{*}, 9^{*}, 10^{*}, 11^{*} \\
12^{*}, 13^{*}, 14^{*}, 15^{*} \\
16^{*}, 17^{*}, 18^{*}, 19^{*} \\
20^{\mathbf{4}}, 21^{*}, 22^{*}, 23^{*}\end{array}$ & \\
\hline 70 & $\begin{array}{c}\text { Dodonaea viscosa }(\mathrm{L} .) \\
\text { Jacq./ Sapindaceae } \\
\text { Sanatha } \\
\text { SAN-SR-204 }\end{array}$ & $\begin{array}{l}\text { Shrub/ } \\
\text { W }\end{array}$ & $\begin{array}{l}\text { Twigs, Leaves/ } \\
\text { Infusion, raw }\end{array}$ & $\begin{array}{l}\text { Skin problems, } \\
\text { toothache, oral problems }\end{array}$ & 3 & 0.25 & 12 & 0.03 & 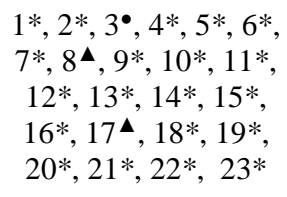 & \\
\hline
\end{tabular}

APPLIED ECOLOGY AND ENVIRONMENTAL RESEARCH 17(2):4725-4805.

http://www.aloki.hu • ISSN 15891623 (Print) • ISSN 17850037 (Online)

DOI: http://dx.doi.org/10.15666/aeer/1702_47254805

(c) 2019, ALÖKI Kft., Budapest, Hungary 


\begin{tabular}{|c|c|c|c|c|c|c|c|c|c|c|}
\hline $\begin{array}{l}\text { Sr. } \\
\text { No. }\end{array}$ & $\begin{array}{c}\text { Species/ Family } \\
\text { Name/ Vernacular } \\
\text { Name/ Voucher } \\
\text { number } \\
\end{array}$ & $\begin{array}{l}\text { Habit } \\
\text { /Type }\end{array}$ & $\begin{array}{l}\text { Part(s) used/ } \\
\text { Mode of } \\
\text { utilization }\end{array}$ & $\begin{array}{l}\text { Medicinal Value/ } \\
\text { Disease treated }\end{array}$ & $\mathbf{U R}^{*}$ & $\mathbf{U V}^{*}$ & $\mathbf{F C}^{*}$ & RFC $^{*}$ & $\begin{array}{l}\text { Previous reports } \\
\text { for comparison }\end{array}$ & Picture \\
\hline 71 & $\begin{array}{l}\text { Echinops echinatus } \\
\text { Roxb./ Asteraceae } \\
\text { SAN-SR-130 }\end{array}$ & $\begin{array}{l}\text { Herb/ } \\
\text { W }\end{array}$ & $\begin{array}{l}\text { Whole plant/ } \\
\text { Decoction }\end{array}$ & $\begin{array}{l}\text { Hepatitis, vomiting, } \\
\text { nausea }\end{array}$ & 3 & 0.2 & 15 & 0.04 & $\begin{array}{c}1^{*}, 2^{*}, 3^{*}, 4^{*}, 5^{*}, 6^{*}, \\
7^{*}, 8^{*}, 9^{*}, 10^{*}, 11^{*}, \\
12^{*}, 13^{*}, 14^{*}, 15^{*}, \\
16^{*}, 17^{*}, 18^{*}, 19^{*} \\
20^{*}, 21^{*}, 22^{*}, 23^{*}\end{array}$ & \\
\hline 72 & $\begin{array}{c}\text { Eclipta prostrata } \\
\text { (L.)L./ Asteraceae } \\
\text { Bhangra } \\
\text { SAN-SR-132 }\end{array}$ & $\begin{array}{c}\text { Herb/ } \\
\text { W }\end{array}$ & $\begin{array}{l}\text { Aerial parts/ } \\
\text { Decoction }\end{array}$ & $\begin{array}{l}\text { Jaundice, cooling effect, } \\
\text { constipation }\end{array}$ & 3 & 0.33 & 9 & 0.03 & $\begin{array}{c}1^{*}, 2^{*}, 3^{*}, 4^{*}, 5^{*}, 6^{*} \\
7^{*}, 8^{*}, 9^{*}, 10^{*}, 11^{*} \\
12^{*}, 13^{*}, 14^{*}, 15^{*} \\
16^{*}, 17^{*}, 18^{\star}, 19^{*} \\
20^{*}, 21^{*}, 22^{*}, 23^{*}\end{array}$ & \\
\hline 73 & $\begin{array}{c}\text { Ehretia obtusifolia } \\
\text { Hochst. ex A. DC./ } \\
\text { Boraginaceae } \\
\text { SAN-SR-36 }\end{array}$ & $\begin{array}{c}\text { Shrub/ } \\
\text { W }\end{array}$ & $\begin{array}{l}\text { Seeds, leaves/ } \\
\text { Infusion, } \\
\text { decoction }\end{array}$ & $\begin{array}{l}\text { Fever, liver problems, } \\
\text { body pain }\end{array}$ & 3 & 0.2 & 15 & 0.04 & $\begin{array}{c}1^{*}, 2^{*}, 3^{*}, 4^{*}, 5^{*}, 6^{*}, \\
7^{*}, 8^{*}, 9^{*}, 10^{*}, 11^{*} \\
12^{*}, 13^{*}, 14^{*}, 15^{*} \\
16^{*}, 17^{*}, 18^{*}, 19^{*} \\
20^{*}, 21^{*}, 22^{*}, 23^{*}\end{array}$ & \\
\hline 74 & $\begin{array}{c}\text { Eichhornia crassipes } \\
\text { (Mart.) Solms/ } \\
\text { Pontederiaceae } \\
\text { Gul-e-rana } \\
\text { SAN-SR-74 }\end{array}$ & $\begin{array}{l}\text { Herb/ } \\
\text { W }\end{array}$ & $\begin{array}{l}\text { Flowers/ } \\
\text { Infusion }\end{array}$ & $\begin{array}{l}\text { Malaria, fever, body } \\
\text { pain }\end{array}$ & 3 & 0.3 & 10 & 0.03 & $\begin{array}{c}1^{*}, 2^{*}, 3^{*}, 4^{*}, 5^{*}, 6^{*}, \\
7^{*}, 8^{*}, 9^{*}, 10^{*}, 11^{*} \\
12^{*}, 13^{*}, 14^{*}, 15^{*} \\
16^{*}, 17^{*}, 18^{*}, 19^{*} \\
20^{*}, 21^{*}, 22^{*}, 23^{*}\end{array}$ & \\
\hline
\end{tabular}

APPLIED ECOLOGY AND ENVIRONMENTAL RESEARCH 17(2):4725-4805.

http://www.aloki.hu • ISSN 15891623 (Print) • ISSN 17850037 (Online)

DOI: http://dx.doi.org/10.15666/aeer/1702_47254805

(c) 2019, ALÖKI Kft., Budapest, Hungary 


\begin{tabular}{|c|c|c|c|c|c|c|c|c|c|c|}
\hline $\begin{array}{l}\text { Sr. } \\
\text { No. }\end{array}$ & $\begin{array}{c}\text { Species/ Family } \\
\text { Name/ Vernacular } \\
\text { Name/ Voucher } \\
\text { number } \\
\end{array}$ & $\begin{array}{l}\text { Habit } \\
\text { /Type }\end{array}$ & $\begin{array}{l}\text { Part(s) used/ } \\
\text { Mode of } \\
\text { utilization }\end{array}$ & $\begin{array}{l}\text { Medicinal Value/ } \\
\text { Disease treated }\end{array}$ & $\mathbf{U R}^{*}$ & $\mathbf{U V}^{*}$ & $\mathbf{F C}^{*}$ & RFC $^{*}$ & $\begin{array}{l}\text { Previous reports } \\
\text { for comparison }^{* *}\end{array}$ & Picture \\
\hline 75 & $\begin{array}{l}\text { Ephedra foliata Boiss. } \\
\text { ex C.A.Mey. sy, } \\
\text { Ephedra ciliata Fisch. } \\
\text { \& C.A.May. } \\
\text { Ephedraceae } \\
\text { SAN-SR-92 }\end{array}$ & $\begin{array}{l}\text { Shrub/ } \\
\text { W }\end{array}$ & $\begin{array}{l}\text { Aerial parts/ } \\
\text { Decoction }\end{array}$ & $\begin{array}{l}\text { Asthma, cough, } \\
\text { bronchitis, flu, } \\
\text { headache, sore throat }\end{array}$ & 6 & 0.26 & 23 & 0.06 & $\begin{array}{c}1^{*}, 2^{*}, 3^{*}, 4^{*}, 5^{*}, 6^{*} \\
7^{*}, 8^{*}, 9^{*}, 10^{*}, 11^{*} \\
12^{*}, 13^{*}, 14^{*}, 15^{*} \\
16^{*}, 17^{*}, 18^{*}, 19^{*} \\
20^{*}, 21^{*}, 22^{*}, 23^{*}\end{array}$ & \\
\hline 76 & $\begin{array}{c}\text { Epilobium hirsutum } \\
\text { L./ Onagraceae } \\
\text { SAN-SR-120 }\end{array}$ & $\begin{array}{c}\text { Herb/ } \\
\text { W }\end{array}$ & Leaves/ Infusion & $\begin{array}{l}\text { Eczema, skin allergy, } \\
\text { wound healing }\end{array}$ & 3 & 0.33 & 9 & 0.02 & $\begin{array}{c}1^{*}, 2^{*}, 3^{*}, 4^{*}, 5^{*}, 6^{*}, \\
7^{*}, 8^{*}, 9^{*}, 10^{*}, 11^{*} \\
12^{*}, 13^{*}, 14^{*}, 15^{*} \\
16^{*}, 17^{*}, 18^{*}, 19^{*} \\
20^{*}, 21^{*}, 22^{*}, 23^{*}\end{array}$ & \\
\hline 77 & $\begin{array}{c}\text { Equisetum arvense L./ } \\
\text { Equisetaceae } \\
\text { Naarri } \\
\text { SAN-SR-34 }\end{array}$ & $\begin{array}{l}\text { Herb/ } \\
\text { W }\end{array}$ & $\begin{array}{l}\text { Whole plant/ } \\
\text { Paste, infusion }\end{array}$ & $\begin{array}{c}\text { Epistaxis, diabetes, skin } \\
\text { problems }\end{array}$ & 3 & 0.33 & 9 & 0.02 & $\begin{array}{c}1^{*}, 2^{\mathbf{\Lambda}}, 3^{*}, 4^{*}, 5^{*}, 6^{*} \\
7^{\mathbf{4}}, 8^{*}, 9^{*}, 10^{*}, 11^{*} \\
12^{\mathbf{4}}, 13^{*}, 14^{*}, 15^{*} \\
16^{*}, 17^{*}, 18^{*}, 19^{*} \\
20^{*}, 21^{*}, 22^{*}, 2^{*}\end{array}$ & \\
\hline
\end{tabular}




\begin{tabular}{|c|c|c|c|c|c|c|c|c|c|c|}
\hline $\begin{array}{l}\text { Sr. } \\
\text { No. }\end{array}$ & $\begin{array}{c}\text { Species/ Family } \\
\text { Name/ Vernacular } \\
\text { Name/ Voucher } \\
\text { number } \\
\end{array}$ & $\begin{array}{l}\text { Habit } \\
\text { /Type }\end{array}$ & $\begin{array}{l}\text { Part(s) used/ } \\
\text { Mode of } \\
\text { utilization }\end{array}$ & $\begin{array}{l}\text { Medicinal Value/ } \\
\text { Disease treated }\end{array}$ & $\mathbf{U} \mathbf{R}^{*}$ & $\mathbf{U V}^{*}$ & $\mathbf{F C}^{*}$ & RFC $^{*}$ & $\begin{array}{l}\text { Previous reports } \\
\text { for comparison }\end{array}$ & Picture \\
\hline 78 & $\begin{array}{c}\text { Eruca sativa Mill./ } \\
\text { Brassicaceae } \\
\text { Jhamayon } \\
\text { SAN-SR-152 }\end{array}$ & $\begin{array}{l}\text { Herb/ } \\
\text { W,C }\end{array}$ & Seeds/ Oil & $\begin{array}{c}\text { Anti-lice, hair tonic, } \\
\text { anti-allergic, } \\
\text { inflammation, dandruff, } \\
\text { itching }\end{array}$ & 6 & 0.17 & 35 & 0.1 & $\begin{array}{c}1^{*}, 2^{*}, 3^{*}, 4^{*}, 5^{*}, 6^{*} \\
7^{*}, 8^{\mathbf{4}}, 9^{*}, 10^{*}, 11^{*} \\
12^{*}, 13^{*}, 14^{*}, 15^{*} \\
16^{*}, 17^{*}, 18^{*}, 19^{*} \\
20^{*}, 21^{*}, 22^{*}, 23^{*}\end{array}$ & \\
\hline 79 & $\begin{array}{c}\text { Eucalyptus } \\
\text { camaldulensis Dehnh./ } \\
\text { Myrtaceae } \\
\text { Sufeda } \\
\text { SAN-SR-15 }\end{array}$ & $\begin{array}{l}\text { Tree/ } \\
\text { C }\end{array}$ & $\begin{array}{l}\text { Leaves/ } \\
\text { Decoction }\end{array}$ & $\begin{array}{l}\text { Respiratory disorders, } \\
\text { febrifuge, malaria, } \\
\text { insect repellent, } \\
\text { vomiting, nausea, }\end{array}$ & 6 & 0.21 & 29 & 0.08 & 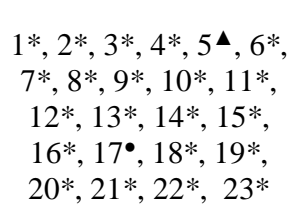 & \\
\hline 80 & $\begin{array}{l}\text { Euphorbia helioscopia } \\
\text { L./ Euphorbiaceae } \\
\text { Chhatri dodak } \\
\text { SAN-SR-04 }\end{array}$ & $\begin{array}{c}\text { Herb/ } \\
\text { W }\end{array}$ & Leaves/ Latex & Warts & 1 & 0.33 & 3 & 0.01 & $\begin{array}{c}1^{*}, 2^{*}, 3^{*}, 4^{*}, 5^{*}, 6^{*}, \\
7^{*}, 8^{\mathbf{4}}, 9^{*}, 10^{*}, 11^{*} \\
12^{*}, 13^{*}, 14^{*}, 15^{*} \\
16^{*}, 17^{*}, 18^{*}, 19^{*} \\
20^{*}, 21^{*}, 22^{*}, 23^{*}\end{array}$ & \\
\hline
\end{tabular}

APPLIED ECOLOGY AND ENVIRONMENTAL RESEARCH 17(2):4725-4805.

http://www.aloki.hu • ISSN 15891623 (Print) • ISSN 17850037 (Online)

DOI: http://dx.doi.org/10.15666/aeer/1702_47254805

(c) 2019, ALÖKI Kft., Budapest, Hungary 


\begin{tabular}{|c|c|c|c|c|c|c|c|c|c|c|}
\hline $\begin{array}{l}\text { Sr. } \\
\text { No. }\end{array}$ & $\begin{array}{c}\text { Species/ Family } \\
\text { Name/ Vernacular } \\
\text { Name/ Voucher } \\
\text { number } \\
\end{array}$ & $\begin{array}{l}\text { Habit } \\
\text { /Type }\end{array}$ & $\begin{array}{l}\text { Part(s) used/ } \\
\text { Mode of } \\
\text { utilization }\end{array}$ & $\begin{array}{l}\text { Medicinal Value/ } \\
\text { Disease treated }\end{array}$ & $\mathbf{U} \mathbf{R}^{*}$ & $\mathbf{U V}^{*}$ & FC $^{*}$ & RFC $^{*}$ & $\begin{array}{l}\text { Previous reports } \\
\text { for comparison** }\end{array}$ & Picture \\
\hline 81 & $\begin{array}{c}\text { Euphorbia } \\
\text { heterophylla L./ } \\
\text { Euphorbiaceae } \\
\text { SAN-SR-65 }\end{array}$ & $\begin{array}{l}\text { Herb/ } \\
\text { W }\end{array}$ & $\begin{array}{l}\text { Whole plant/ } \\
\text { Infusion, paste }\end{array}$ & Scabies, warts, & 2 & 0.66 & 3 & 0.01 & $\begin{array}{c}1^{*}, 2^{*}, 3^{*}, 4^{*}, 5^{*}, 6^{*}, \\
7^{*}, 8^{*}, 9^{*}, 10^{*}, 11^{*}, \\
12^{*}, 13^{*}, 14^{*}, 15^{*} \\
16^{*}, 17^{*}, 18^{*}, 19^{*} \\
20^{*}, 21^{*}, 22^{*}, 23^{*}\end{array}$ & \\
\hline 82 & $\begin{array}{c}\text { Euphorbia hirta L./ } \\
\text { Euphorbiaceae } \\
\text { SAN-SR-154 }\end{array}$ & $\begin{array}{l}\text { Herb/ } \\
\text { W }\end{array}$ & $\begin{array}{l}\text { Whole plant/ } \\
\text { Decoction }\end{array}$ & $\begin{array}{l}\text { Cough, obesity, } \\
\text { constipation, blood } \\
\text { purifier }\end{array}$ & 4 & 0.36 & 11 & 0.03 & $\begin{array}{c}1^{\bullet}, 2^{*}, 3^{*}, 4^{*}, 5^{*}, 6^{*}, \\
7^{*}, 8^{*}, 9^{*}, 10^{*}, 11^{*} \\
12^{*}, 13^{*}, 14^{*}, 15^{*} \\
16^{*}, 17^{*}, 18^{*}, 19^{*} \\
20^{*}, 21^{*}, 22^{*}, 23^{*}\end{array}$ & \\
\hline 83 & $\begin{array}{l}\text { Euphorbia peplus L./ } \\
\text { Euphorbiaceae } \\
\text { SAN-SR-177 }\end{array}$ & $\begin{array}{l}\text { Herb/ } \\
\text { W }\end{array}$ & $\begin{array}{l}\text { Leaves/ Latex, } \\
\text { infusion }\end{array}$ & $\begin{array}{l}\text { Skin problems, wound } \\
\text { healing, blood purifier, } \\
\text { anti-allergic }\end{array}$ & 4 & 0.25 & 16 & 0.04 & $\begin{array}{c}1^{*}, 2^{*}, 3^{*}, 4^{*}, 5^{*}, 6^{*} \\
7^{*}, 8^{*}, 9^{*}, 10^{*}, 11^{*} \\
12^{*}, 13^{*}, 14^{*}, 15^{*} \\
16^{*}, 17^{*}, 18^{*}, 19^{*} \\
20^{*}, 21^{*}, 22^{*}, 23^{*}\end{array}$ & \\
\hline 84 & $\begin{array}{c}\text { Euphorbia prostrata } \\
\text { Aiton/ Euphorbiaceae } \\
\text { SAN-SR-206 }\end{array}$ & $\begin{array}{l}\text { Herb/ } \\
\text { W }\end{array}$ & $\begin{array}{l}\text { Leaves/ } \\
\text { Decoction }\end{array}$ & $\begin{array}{l}\text { Piles, constipation, } \\
\text { gynecological problems }\end{array}$ & 3 & 0.5 & 6 & 0.02 & 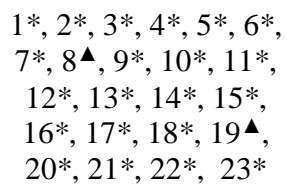 & \\
\hline
\end{tabular}

APPLIED ECOLOGY AND ENVIRONMENTAL RESEARCH 17(2):4725-4805.

http://www.aloki.hu • ISSN 15891623 (Print) • ISSN 17850037 (Online)

DOI: http://dx.doi.org/10.15666/aeer/1702_47254805

๔ 2019, ALÖKI Kft., Budapest, Hungary 


\begin{tabular}{|c|c|c|c|c|c|c|c|c|c|c|}
\hline $\begin{array}{l}\text { Sr. } \\
\text { No. }\end{array}$ & $\begin{array}{c}\text { Species/ Family } \\
\text { Name/ Vernacular } \\
\text { Name/ Voucher } \\
\text { number } \\
\end{array}$ & $\begin{array}{l}\text { Habit } \\
\text { /Type }\end{array}$ & $\begin{array}{l}\text { Part(s) used/ } \\
\text { Mode of } \\
\text { utilization }\end{array}$ & $\begin{array}{l}\text { Medicinal Value/ } \\
\text { Disease treated }\end{array}$ & $\mathbf{U} \mathbf{R}^{*}$ & $\mathbf{U V} \mathbf{V}^{*}$ & $\mathbf{F C}^{*}$ & RFC $^{*}$ & $\begin{array}{l}\text { Previous reports } \\
\text { for comparison }\end{array}$ & Picture \\
\hline 85 & $\begin{array}{c}\text { Euphorbia serpens } \\
\text { Euphorbiaceae } \\
\text { SAN-SR-171 }\end{array}$ & $\begin{array}{c}\text { Herb/ } \\
\text { W }\end{array}$ & $\begin{array}{l}\text { Whole plant/ } \\
\text { Decoction }\end{array}$ & $\begin{array}{l}\text { Skin problems, warts, } \\
\text { asthma, cough, }\end{array}$ & 4 & 0.33 & 12 & 0.03 & $\begin{array}{c}1^{*}, 2^{*}, 3^{*}, 4^{*}, 5^{*}, 6^{*} \\
7^{*}, 8^{*}, 9^{*}, 10^{*}, 11^{*} \\
12^{*}, 13^{*}, 14^{*}, 15^{*} \\
16^{*}, 17^{*}, 18^{*}, 19^{*} \\
20^{*}, 21^{*}, 22^{*}, 23^{*}\end{array}$ & \\
\hline 86 & $\begin{array}{c}\text { Evolvulus alsinoides } \\
\text { (L.) L./ } \\
\text { Convolvulaceae } \\
\text { SAN-SR-52 }\end{array}$ & $\begin{array}{l}\text { Herb/ } \\
\text { W }\end{array}$ & $\begin{array}{l}\text { Whole plant/ } \\
\text { Decoction }\end{array}$ & $\begin{array}{l}\text { Obesity, fever, } \\
\text { hypertension }\end{array}$ & 3 & 0.21 & 14 & 0.04 & 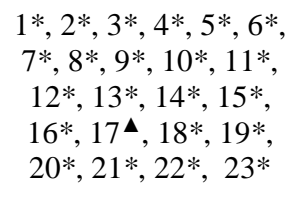 & \\
\hline 87 & $\begin{array}{c}\text { Fagonia indica } \\
\text { Burm.f./ } \\
\text { Zygophyllaceae/ } \\
\text { Dhamasa } \\
\text { SAN-SR-108 }\end{array}$ & $\begin{array}{c}\text { Herb/ } \\
\text { W }\end{array}$ & $\begin{array}{c}\text { Aerial } \\
\text { parts/Decoction }\end{array}$ & $\begin{array}{l}\text { Cooling effect, } \\
\text { vomiting, bloating }\end{array}$ & 3 & 0.3 & 10 & 0.03 & $\begin{array}{c}1^{*}, 2^{*}, 3^{*}, 4^{*}, 5^{*}, 6^{*} \\
7^{*}, 8^{*}, 9^{*}, 10^{*}, 11^{*} \\
12^{*}, 13^{*}, 14^{*}, 15^{*} \\
16^{*}, 17^{*}, 18^{*}, 19^{*} \\
20^{*}, 21^{*}, 22^{*}, 23^{*}\end{array}$ & \\
\hline 88 & $\begin{array}{c}\text { Ficus benghalensis L./ } \\
\text { Moraceae/ } \\
\text { Borrh } \\
\text { SAN-SR-140 }\end{array}$ & $\begin{array}{l}\text { Tree/ } \\
\text { W,C }\end{array}$ & Leaves/ Latex & $\begin{array}{l}\text { Chicken pox, small pox, } \\
\text { piles }\end{array}$ & 3 & 0.14 & 22 & 0.06 & 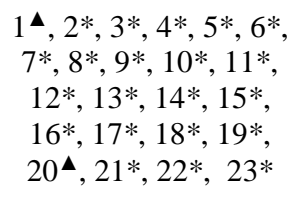 & \\
\hline
\end{tabular}

APPLIED ECOLOGY AND ENVIRONMENTAL RESEARCH 17(2):4725-4805.

http://www.aloki.hu • ISSN 15891623 (Print) • ISSN 17850037 (Online)

DOI: http://dx.doi.org/10.15666/aeer/1702_47254805

(c) 2019, ALÖKI Kft., Budapest, Hungary 


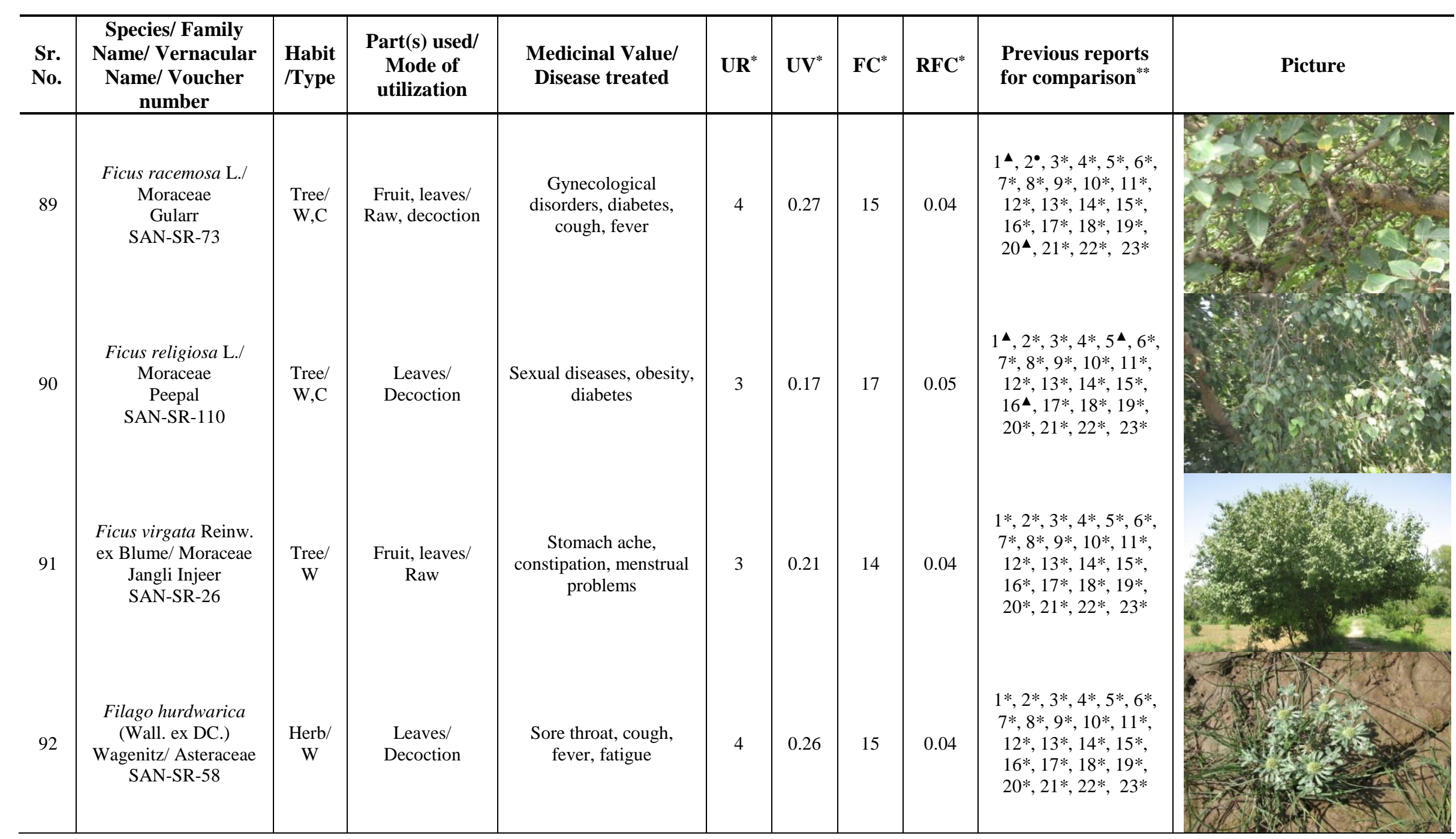

APPLIED ECOLOGY AND ENVIRONMENTAL RESEARCH 17(2):4725-4805.

http://www.aloki.hu • ISSN 15891623 (Print) • ISSN 17850037 (Online)

DOI: http://dx.doi.org/10.15666/aeer/1702_47254805

(c) 2019, ALÖKI Kft., Budapest, Hungary 


\begin{tabular}{|c|c|c|c|c|c|c|c|c|c|c|}
\hline $\begin{array}{l}\text { Sr. } \\
\text { No. }\end{array}$ & $\begin{array}{c}\text { Species/ Family } \\
\text { Name/ Vernacular } \\
\text { Name/ Voucher } \\
\text { number } \\
\end{array}$ & $\begin{array}{l}\text { Habit } \\
\text { /Type }\end{array}$ & $\begin{array}{c}\text { Part(s) used/ } \\
\text { Mode of } \\
\text { utilization }\end{array}$ & $\begin{array}{l}\text { Medicinal Value/ } \\
\text { Disease treated }\end{array}$ & $\mathbf{U R}^{*}$ & $\mathbf{U V} \mathbf{V}^{*}$ & $\mathbf{F C}^{*}$ & RFC $^{*}$ & $\begin{array}{l}\text { Previous reports } \\
\text { for comparison }\end{array}$ & Picture \\
\hline 93 & $\begin{array}{c}\text { Forsskaolea } \\
\text { tenacissima } \text { L./ } \\
\text { Urticaceae } \\
\text { Nettle } \\
\text { SAN-SR-82 }\end{array}$ & $\begin{array}{c}\text { Herb/ } \\
\text { W }\end{array}$ & $\begin{array}{l}\text { Whole plant/ } \\
\text { Decoction }\end{array}$ & $\begin{array}{l}\text { Hypertension, ulcer, } \\
\text { stomach ache }\end{array}$ & 3 & 0.18 & 16 & 0.04 & $\begin{array}{c}1^{*}, 2^{*}, 3^{*}, 4^{*}, 5^{*}, 6^{*}, \\
7^{*}, 8^{*}, 9^{*}, 10^{*}, 11^{*} \\
12^{*}, 13^{*}, 14^{*}, 15^{*} \\
16^{*}, 17^{*}, 18^{*}, 19^{*} \\
20^{*}, 21^{*}, 22^{*}, 23^{*}\end{array}$ & \\
\hline 94 & $\begin{array}{c}\text { Fumaria indica } \\
\text { (Hausskn.) Pugsley/ } \\
\text { Papaveraceae } \\
\text { Paaprra } \\
\text { SAN-SR-135 }\end{array}$ & $\begin{array}{c}\text { Herb/ } \\
\text { W }\end{array}$ & $\begin{array}{l}\text { Flowers/ } \\
\text { Infusion }\end{array}$ & $\begin{array}{l}\text { Depurative, vomiting, } \\
\text { jaundice, stomach ache }\end{array}$ & 4 & 0.44 & 9 & 0.02 & $\begin{array}{c}1^{*}, 2^{\star}, 3^{*}, 4^{*}, 5^{\bullet}, 6^{*} \\
7^{*}, 8^{\mathbf{4}}, 9^{*}, 10^{*}, 11^{\bullet} \\
12^{*}, 13^{*}, 14^{*}, 15^{*} \\
16^{*}, 17^{*}, 18^{*}, 19^{*} \\
20^{*}, 21^{*}, 22^{*}, 23^{*}\end{array}$ & \\
\hline 95 & $\begin{array}{c}\text { Galium aparine L./ } \\
\text { Rubiaceae } \\
\text { SAN-SR-08 }\end{array}$ & $\begin{array}{c}\text { Herb/ } \\
\text { W }\end{array}$ & $\begin{array}{l}\text { Whole plant/ } \\
\text { Tea, infusion } \\
\text { decoction }\end{array}$ & $\begin{array}{c}\text { Obesity, hypertension, } \\
\text { skin problems, body } \\
\text { pain }\end{array}$ & 4 & 0.26 & 15 & 0.04 & $\begin{array}{c}1^{*}, 2^{*}, 3^{*}, 4^{\mathbf{4}}, 5^{*}, 6^{*} \\
7^{*}, 8^{*}, 9^{*}, 10^{*}, 11^{*} \\
12^{\mathbf{4}}, 13^{*}, 14^{*}, 15^{*} \\
16^{*}, 17^{*}, 18^{*}, 19^{*} \\
20^{*}, 21^{*}, 22^{*}, 23^{*}\end{array}$ & \\
\hline
\end{tabular}

APPLIED ECOLOGY AND ENVIRONMENTAL RESEARCH 17(2):4725-4805.

http://www.aloki.hu • ISSN 15891623 (Print) • ISSN 17850037 (Online)

DOI: http://dx.doi.org/10.15666/aeer/1702_47254805

(c) 2019, ALÖKI Kft., Budapest, Hungary 


\begin{tabular}{|c|c|c|c|c|c|c|c|c|c|c|}
\hline $\begin{array}{l}\text { Sr. } \\
\text { No. }\end{array}$ & $\begin{array}{c}\text { Species/ Family } \\
\text { Name/ Vernacular } \\
\text { Name/ Voucher } \\
\text { number } \\
\end{array}$ & $\begin{array}{l}\text { Habit } \\
\text { /Type }\end{array}$ & $\begin{array}{l}\text { Part(s) used/ } \\
\text { Mode of } \\
\text { utilization }\end{array}$ & $\begin{array}{l}\text { Medicinal Value/ } \\
\text { Disease treated }\end{array}$ & $\mathbf{U R}^{*}$ & $\mathbf{U V}^{*}$ & $\mathbf{F C}^{*}$ & RFC $^{*}$ & $\begin{array}{l}\text { Previous reports } \\
\text { for comparison** }\end{array}$ & Picture \\
\hline 96 & $\begin{array}{l}\text { Gastrocotyle hispida } \\
\text { (Forssk.) Bunge/ } \\
\text { Boraginaceae } \\
\text { Khatol } \\
\text { SAN-SR-113 }\end{array}$ & $\begin{array}{l}\text { Herb/ } \\
\text { W }\end{array}$ & $\begin{array}{l}\text { Leaves/ } \\
\text { Decoction }\end{array}$ & $\begin{array}{l}\text { Purgative, constipation, } \\
\text { vomiting }\end{array}$ & 3 & 0.37 & 8 & 0.02 & $\begin{array}{c}1^{*}, 2^{*}, 3^{*}, 4^{*}, 5^{*}, 6^{*}, \\
7^{*}, 8^{*}, 9^{*}, 10^{*}, 11^{*}, \\
12^{*}, 13^{*}, 14^{*}, 15^{*}, \\
16^{*}, 17^{*}, 18^{*}, 19^{*}, \\
20^{*}, 21^{*}, 22^{*}, 23^{*}\end{array}$ & \\
\hline 97 & $\begin{array}{c}\text { Geranium mascatense } \\
\text { Boiss./ Geraniaceae } \\
\text { SAN-SR-129 }\end{array}$ & $\begin{array}{l}\text { Herb/ } \\
\mathrm{W}\end{array}$ & $\begin{array}{l}\text { Aerial parts/ } \\
\text { Decoction }\end{array}$ & $\begin{array}{l}\text { Epilepsy, back ache, } \\
\text { joint pain, }\end{array}$ & 3 & 0.25 & 12 & 0.03 & $\begin{array}{c}1^{*}, 2^{*}, 3^{*}, 4^{*}, 5^{*}, 6^{*}, \\
7^{*}, 8^{*}, 9^{*}, 10^{*}, 11^{*}, \\
12^{*}, 13^{*}, 14^{*}, 15^{*}, \\
16^{*}, 17^{*}, 18^{*}, 19^{*}, \\
20^{*}, 21^{*}, 22^{*}, 23^{*}\end{array}$ & \\
\hline 98 & $\begin{array}{c}\text { Glandularia pulchella } \\
\text { (Sweet) Tronc. (Syn. } \\
\text { Verbena tenisecta } \\
\text { Briq.)/ Verbenaceae } \\
\text { SAN-SR-136 }\end{array}$ & $\begin{array}{l}\text { Herb/ } \\
\text { W }\end{array}$ & $\begin{array}{l}\text { Whole plant/ } \\
\text { Infusion }\end{array}$ & $\begin{array}{l}\text { Hypertension, anxiety, } \\
\text { fever }\end{array}$ & 3 & 0.43 & 7 & 0.02 & $\begin{array}{c}1^{*}, 2^{*}, 3^{*}, 4^{*}, 5^{*}, 6^{*}, \\
7^{*}, 8^{*}, 9^{*}, 10^{*}, 11^{*}, \\
12^{*}, 13^{*}, 14^{*}, 15^{*}, \\
16^{*}, 17^{*}, 18^{*}, 19^{*}, \\
20^{*}, 21^{*}, 22^{*}, 23^{*}\end{array}$ & \\
\hline 99 & $\begin{array}{c}\text { Grewia optiva } \\
\text { J.R.Drumm. ex Burret/ } \\
\text { Malvaceae } \\
\text { SAN-SR-143 }\end{array}$ & $\begin{array}{c}\text { Tree/ } \\
\text { W }\end{array}$ & Fruit/ Raw & $\begin{array}{l}\text { Jaundice, digestive } \\
\text { problems, tonic }\end{array}$ & 3 & 0.15 & 20 & 0.06 & $\begin{array}{c}1^{*}, 2^{\star}, 3^{*}, 4^{*}, 5^{*}, 6^{*}, \\
7^{*}, 8^{*}, 9^{*}, 10^{*}, 11^{*}, \\
12^{*}, 13^{*}, 14^{*}, 15^{*}, \\
16^{*}, 17^{*}, 18^{*}, 19^{*}, \\
20^{*}, 21^{*}, 22^{*}, 23^{*}\end{array}$ & \\
\hline
\end{tabular}

APPLIED ECOLOGY AND ENVIRONMENTAL RESEARCH 17(2):4725-4805.

http://www.aloki.hu • ISSN 15891623 (Print) • ISSN 17850037 (Online)

DOI: http://dx.doi.org/10.15666/aeer/1702_47254805

(c) 2019, ALÖKI Kft., Budapest, Hungary 


\begin{tabular}{|c|c|c|c|c|c|c|c|c|c|c|}
\hline $\begin{array}{l}\text { Sr. } \\
\text { No. }\end{array}$ & $\begin{array}{c}\text { Species/ Family } \\
\text { Name/ Vernacular } \\
\text { Name/ Voucher } \\
\text { number } \\
\end{array}$ & $\begin{array}{l}\text { Habit } \\
\text { /Type }\end{array}$ & $\begin{array}{l}\text { Part(s) used/ } \\
\text { Mode of } \\
\text { utilization }\end{array}$ & $\begin{array}{l}\text { Medicinal Value/ } \\
\text { Disease treated }\end{array}$ & $\mathbf{U R}^{*}$ & $\mathbf{U V}^{*}$ & $\mathbf{F C}^{*}$ & RFC $^{*}$ & $\begin{array}{l}\text { Previous reports } \\
\text { for comparison }\end{array}$ & Picture \\
\hline 100 & $\begin{array}{c}\text { Grewia tenax (Forssk.) } \\
\text { Fiori/ Malvaceae } \\
\text { Gungair } \\
\text { SAN-SR-150 }\end{array}$ & $\begin{array}{l}\text { Shrub/ } \\
\text { W }\end{array}$ & Fruit/ Raw & $\begin{array}{l}\text { Jaundice, digestive } \\
\text { problems, tonic }\end{array}$ & 3 & 0.15 & 20 & 0.06 & $\begin{array}{c}1^{*}, 2^{*}, 3^{*}, 4^{*}, 5^{*}, 6^{*} \\
7^{*}, 8^{*}, 9^{*}, 10^{*}, 11^{*} \\
12^{*}, 13^{*}, 14^{*}, 15^{*} \\
16^{*}, 17^{\star}, 18^{*}, 19^{*} \\
20^{*}, 21^{*}, 22^{*}, 23^{*}\end{array}$ & \\
\hline 101 & $\begin{array}{l}\text { Grewia villosa Willd./ } \\
\text { Tiliaceae SAN-SR-156 }\end{array}$ & $\begin{array}{c}\text { Shrub/ } \\
\text { W }\end{array}$ & Fruit/ Raw & $\begin{array}{l}\text { Jaundice, digestive } \\
\text { problems, tonic }\end{array}$ & 3 & 0.15 & 20 & 0.06 & 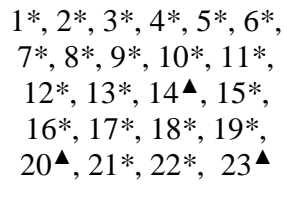 & \\
\hline 102 & $\begin{array}{c}\text { Gymnosporia spinosa } \\
\text { (Blanco) Merr. \& } \\
\text { Rolfe/ Celastraceae } \\
\text { Pattassi } \\
\text { SAN-SR-162 }\end{array}$ & $\begin{array}{c}\text { Shrub/ } \\
\text { W }\end{array}$ & Seeds/ Ash & $\begin{array}{l}\text { Toothache, oral } \\
\text { infections }\end{array}$ & 2 & 0.33 & 6 & 0.02 & $\begin{array}{c}1^{*}, 2^{*}, 3^{*}, 4^{*}, 5^{*}, 6^{*}, \\
7^{*}, 8^{*}, 9^{*}, 10^{*}, 11^{*}, \\
12^{*}, 13^{*}, 14^{*}, 15^{*}, \\
16^{*}, 17^{*}, 18^{*}, 19^{*}, \\
2^{*}, 21^{*}, 22^{*}, 23^{*}\end{array}$ & \\
\hline 103 & $\begin{array}{c}\text { Gynandropsis } \\
\text { gynandra (L.) Briq./ } \\
\text { Cleomaceae } \\
\text { Badal banga } \\
\text { SAN-SR-11 }\end{array}$ & $\begin{array}{l}\text { Herb/ } \\
\text { W }\end{array}$ & $\begin{array}{l}\text { Aerial parts/ } \\
\text { Juice }\end{array}$ & $\begin{array}{l}\text { Epilepsy, menstrual } \\
\text { pain, vomiting }\end{array}$ & 3 & 0.33 & 9 & 0.02 & $\begin{array}{c}1^{*}, 2^{*}, 3^{*}, 4^{*}, 5^{*}, 6^{*}, \\
7^{*}, 8^{*}, 9^{*}, 10^{*}, 11^{*}, \\
12^{*}, 13^{*}, 14^{*}, 15^{*}, \\
16^{*}, 17^{*}, 18^{*}, 19^{*} \\
20^{*}, 21^{*}, 22^{*}, 23^{*}\end{array}$ & \\
\hline
\end{tabular}

APPLIED ECOLOGY AND ENVIRONMENTAL RESEARCH 17(2):4725-4805.

http://www.aloki.hu • ISSN 15891623 (Print) • ISSN 17850037 (Online)

DOI: http://dx.doi.org/10.15666/aeer/1702_47254805

(c) 2019, ALÖKI Kft., Budapest, Hungary 


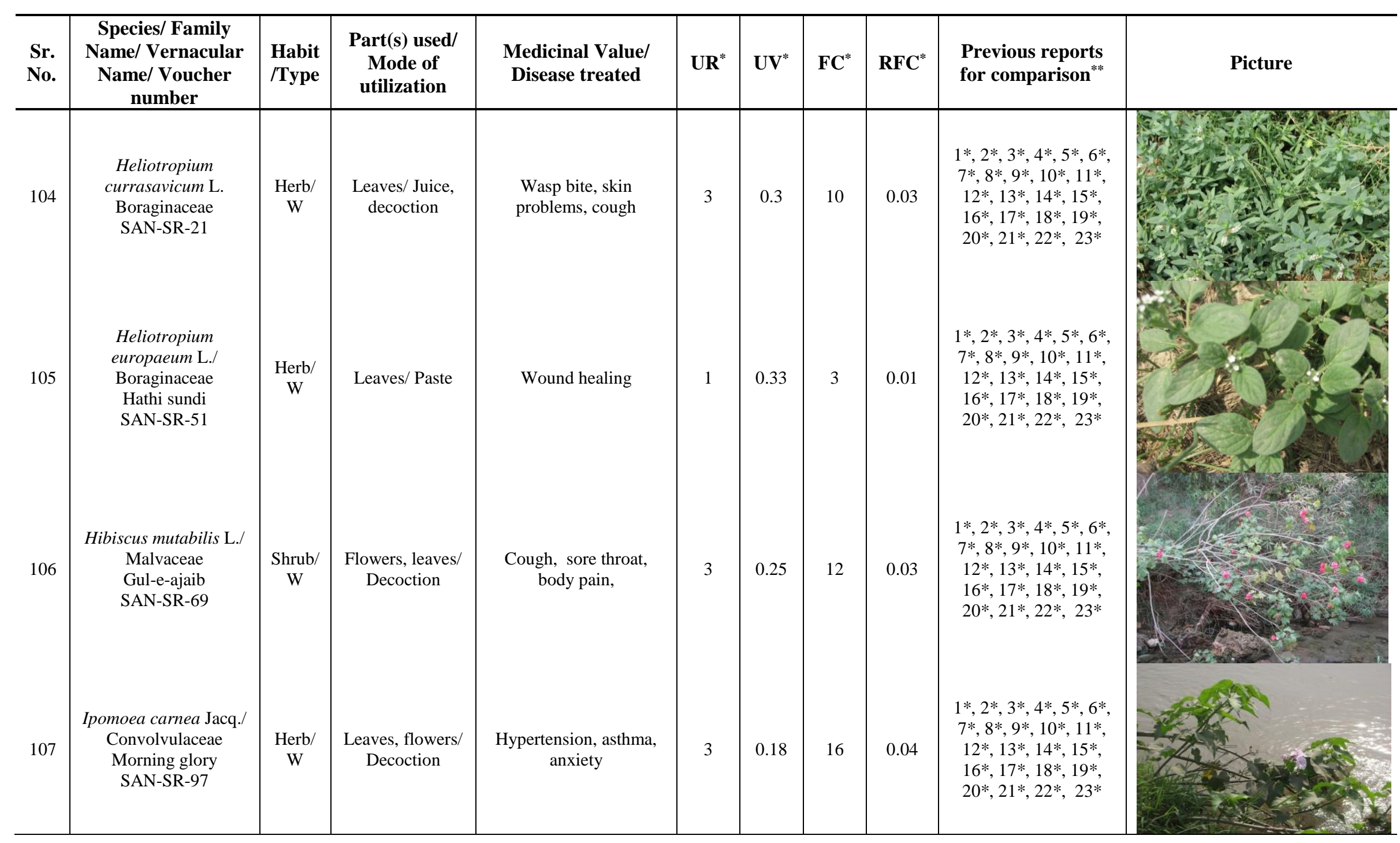

APPLIED ECOLOGY AND ENVIRONMENTAL RESEARCH 17(2):4725-4805.

http://www.aloki.hu • ISSN 15891623 (Print) • ISSN 17850037 (Online)

DOI: http://dx.doi.org/10.15666/aeer/1702_47254805

(๖) 2019, ALÖKI Kft., Budapest, Hungary 


\begin{tabular}{|c|c|c|c|c|c|c|c|c|c|c|}
\hline $\begin{array}{l}\text { Sr. } \\
\text { No. }\end{array}$ & $\begin{array}{c}\text { Species/ Family } \\
\text { Name/ Vernacular } \\
\text { Name/ Voucher } \\
\text { number } \\
\end{array}$ & $\begin{array}{l}\text { Habit } \\
\text { /Type }\end{array}$ & $\begin{array}{l}\text { Part(s) used/ } \\
\text { Mode of } \\
\text { utilization }\end{array}$ & $\begin{array}{l}\text { Medicinal Value/ } \\
\text { Disease treated }\end{array}$ & $\mathbf{U} \mathbf{R}^{*}$ & $\mathbf{U V}^{*}$ & $\mathbf{F C}^{*}$ & RFC $^{*}$ & $\begin{array}{l}\text { Previous reports } \\
\text { for comparison }^{* *}\end{array}$ & Picture \\
\hline 108 & $\begin{array}{c}\text { Juncus elegans Royle } \\
\text { ex Sam./ Juncaceae } \\
\text { Water Dila } \\
\text { SAN-SR-78 }\end{array}$ & $\begin{array}{l}\text { Herb/ } \\
\text { W }\end{array}$ & ----- & ----- & 0 & 0 & 15 & 0.04 & $\begin{array}{c}1,2,3,4,5,6,7,8,9 \\
10,11,12,13,14,15 \\
16,17,18,19,20,21 \\
22,23\end{array}$ & \\
\hline 109 & $\begin{array}{c}\text { Justicia adhatoda } \text { L./ } \\
\text { Acanthaceae } \\
\text { Bhaikarr } \\
\text { SAN-SR-20 }\end{array}$ & $\begin{array}{l}\text { Herb/ } \\
\text { W }\end{array}$ & $\begin{array}{l}\text { Flower/ } \\
\text { Decoction }\end{array}$ & $\begin{array}{l}\text { Cold, cough, body pain, } \\
\text { headache }\end{array}$ & 4 & 0.2 & 20 & 0.06 & 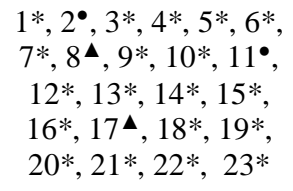 & \\
\hline 110 & $\begin{array}{c}\text { Kickxia ramosissima } \\
\text { (Wall.) Janch./ } \\
\text { Plantaginaceae } \\
\text { SAN-SR-40 }\end{array}$ & $\begin{array}{c}\text { Herb/ } \\
\text { W }\end{array}$ & $\begin{array}{c}\text { Whole plant/ } \\
\text { Decoction, paste }\end{array}$ & $\begin{array}{l}\text { Febrifuge, body pain, } \\
\text { inflammation }\end{array}$ & 3 & 0.37 & 8 & 0.02 & $\begin{array}{c}1^{*}, 2^{*}, 3^{*}, 4^{*}, 5^{*}, 6^{*}, \\
7^{*}, 8^{*}, 9^{*}, 10^{*}, 11^{*} \\
12^{*}, 13^{*}, 14^{*}, 15^{*} \\
16^{*}, 17^{*}, 18^{*}, 19^{*} \\
20^{*}, 21^{*}, 22^{*}, 23^{*}\end{array}$ & \\
\hline
\end{tabular}




\begin{tabular}{|c|c|c|c|c|c|c|c|c|c|c|}
\hline $\begin{array}{l}\text { Sr. } \\
\text { No. }\end{array}$ & $\begin{array}{c}\text { Species/ Family } \\
\text { Name/ Vernacular } \\
\text { Name/ Voucher } \\
\text { number } \\
\end{array}$ & $\begin{array}{l}\text { Habit } \\
\text { /Type }\end{array}$ & $\begin{array}{l}\text { Part(s) used/ } \\
\text { Mode of } \\
\text { utilization }\end{array}$ & $\begin{array}{l}\text { Medicinal Value/ } \\
\text { Disease treated }\end{array}$ & $\mathbf{U} \mathbf{R}^{*}$ & $\mathbf{U V}^{*}$ & $\mathbf{F C}^{*}$ & RFC $^{*}$ & $\begin{array}{l}\text { Previous reports } \\
\text { for comparison** }\end{array}$ & Picture \\
\hline 111 & $\begin{array}{c}\text { Lactuca serriola L./ } \\
\text { Asteraceae } \\
\text { SAN-SR-60 }\end{array}$ & $\begin{array}{c}\text { Herb/ } \\
\text { W }\end{array}$ & ----- & ----- & 0 & 0 & 12 & 0.03 & $\begin{array}{c}1^{*}, 2^{*}, 3^{*}, 4^{*}, 5^{*}, 6^{*}, \\
7^{*}, 8^{*}, 9^{*}, 10^{*}, 11^{*}, \\
12^{*}, 13^{*}, 14^{*}, 15^{*} \\
16^{*}, 17^{*}, 18^{*}, 19^{*} \\
20^{*}, 21^{*}, 22^{*}, 23^{*}\end{array}$ & \\
\hline 112 & $\begin{array}{c}\text { Lantana camara L. } \\
\text { /Verbenaceae } \\
\text { Bhang } \\
\text { SAN-SR-86 }\end{array}$ & $\begin{array}{l}\text { Shrub/ } \\
\text { W }\end{array}$ & Leaves/ Infusion & $\begin{array}{l}\text { Carminative, febrifuge, } \\
\text { malaria, cough, cold }\end{array}$ & 5 & 0.26 & 19 & 0.05 & $\begin{array}{c}1^{*}, 2^{*}, 3^{*}, 4^{*}, 5^{*}, 6^{*}, \\
7^{*}, 8^{*}, 9^{*}, 10^{*}, 11^{*}, \\
12^{*}, 13^{*}, 14^{*}, 15^{*} \\
16^{\mathbf{4}}, 17^{\mathbf{4}}, 18^{\mathbf{4}}, 19^{*} \\
20^{\mathbf{4}}, 21^{*}, 22^{*}, 23^{*}\end{array}$ & \\
\hline 113 & $\begin{array}{c}\text { Lathyrus aphaca L./ } \\
\text { Fabaceae } \\
\text { SAN-SR-121 }\end{array}$ & $\begin{array}{l}\text { Herb/ } \\
\text { W }\end{array}$ & $\begin{array}{l}\text { Leaves, seeds/ } \\
\text { Infusion, raw }\end{array}$ & $\begin{array}{l}\text { Hypertension, insomnia, } \\
\text { fever }\end{array}$ & 3 & 0.23 & 13 & 0.04 & $\begin{array}{c}1^{*}, 2^{\bullet}, 3^{*}, 4^{*}, 5^{\star}, 6^{*}, \\
7^{*}, 8^{*}, 9^{*}, 10^{*}, 11^{*} \\
12^{*}, 13^{*}, 14^{*}, 15^{*} \\
16^{*}, 17^{*}, 18^{*}, 19^{*} \\
20^{*}, 21^{*}, 22^{*}, 23^{*}\end{array}$ & \\
\hline
\end{tabular}




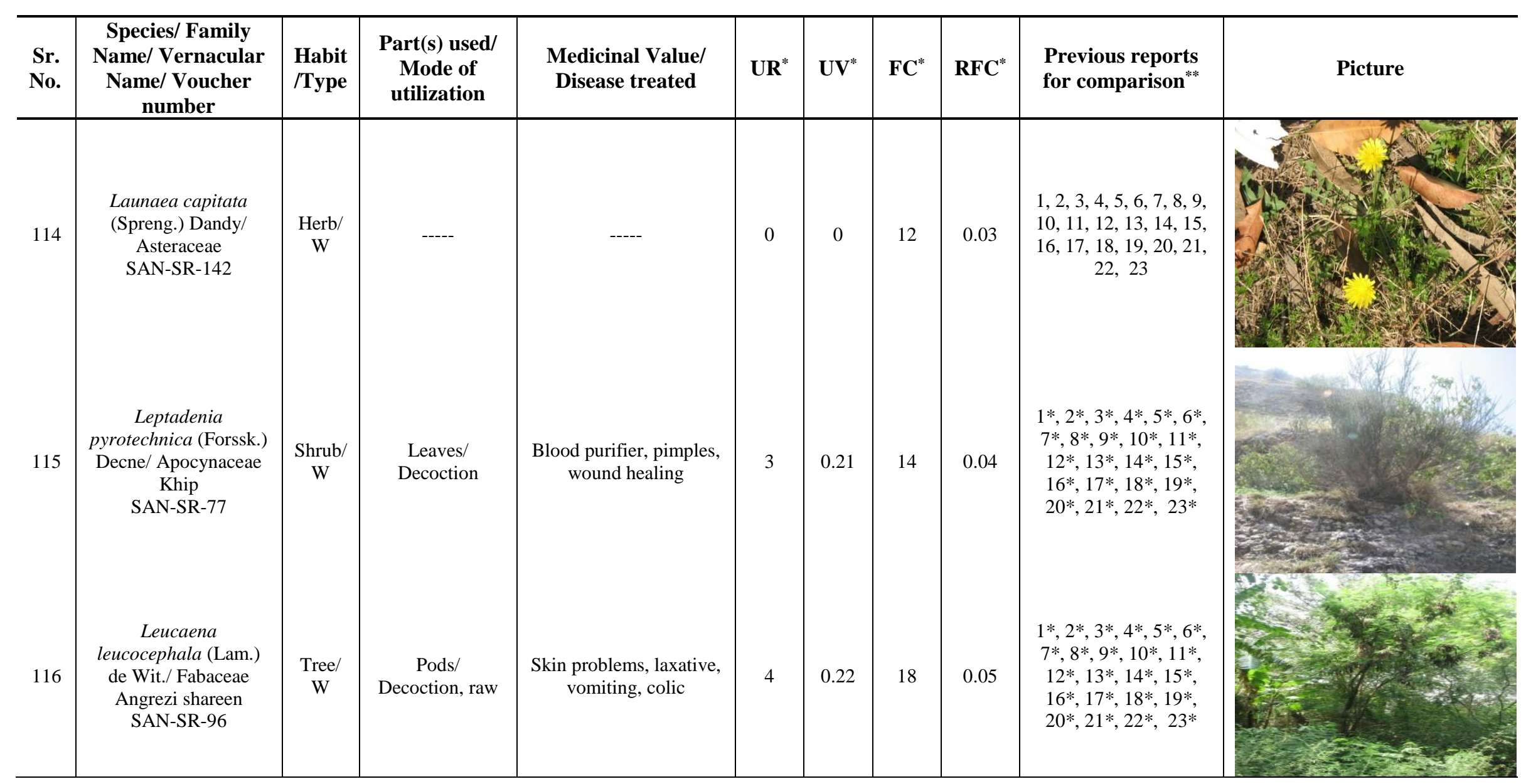




\begin{tabular}{|c|c|c|c|c|c|c|c|c|c|c|}
\hline $\begin{array}{l}\text { Sr. } \\
\text { No. }\end{array}$ & $\begin{array}{c}\text { Species/ Family } \\
\text { Name/ Vernacular } \\
\text { Name/ Voucher } \\
\text { number } \\
\end{array}$ & $\begin{array}{l}\text { Habit } \\
\text { /Type }\end{array}$ & $\begin{array}{l}\text { Part(s) used/ } \\
\text { Mode of } \\
\text { utilization }\end{array}$ & $\begin{array}{l}\text { Medicinal Value/ } \\
\text { Disease treated }\end{array}$ & $\mathbf{U} \mathbf{R}^{*}$ & $\mathbf{U V}^{*}$ & $\mathbf{F C}^{*}$ & RFC $^{*}$ & $\begin{array}{l}\text { Previous reports } \\
\text { for comparison** }\end{array}$ & Picture \\
\hline 117 & $\begin{array}{c}\text { Lindenbergia } \\
\text { abyssinica } \text { Hochst. ex } \\
\text { Benth./ } \\
\text { Scrophulariaceae } \\
\text { SAN-SR-175 }\end{array}$ & $\begin{array}{l}\text { Herb/ } \\
\text { W }\end{array}$ & $\begin{array}{l}\text { Whole plant/ } \\
\text { Decoction, } \\
\text { infusion, juice }\end{array}$ & $\begin{array}{c}\text { Stomach problems, } \\
\text { epilepsy, body pain, } \\
\text { headache }\end{array}$ & 4 & 0.26 & 15 & 0.04 & $\begin{array}{c}1^{*}, 2^{*}, 3^{*}, 4^{*}, 5^{*}, 6^{*}, \\
7^{*}, 8^{*}, 9^{*}, 10^{*}, 11^{*} \\
12^{*}, 13^{*}, 14^{*}, 15^{*} \\
16^{*}, 17^{*}, 18^{*}, 19^{*} \\
20^{*}, 21^{*}, 22^{*}, 23^{*}\end{array}$ & \\
\hline 118 & $\begin{array}{c}\text { Lindenbergia indica } \\
\text { Vatke/ } \\
\text { Scrophulariaceae } \\
\text { SAN-SR-207 }\end{array}$ & $\begin{array}{l}\text { Herb/ } \\
\text { W }\end{array}$ & $\begin{array}{l}\text { Whole plant/ } \\
\text { Decoction, } \\
\text { infusion, juice }\end{array}$ & $\begin{array}{c}\text { Stomach problems, } \\
\text { epilepsy, body pain, } \\
\text { headache }\end{array}$ & 4 & 0.26 & 15 & 0.04 & $\begin{array}{c}1^{*}, 2^{*}, 3^{*}, 4^{*}, 5^{*}, 6^{*}, \\
7^{*}, 8^{*}, 9^{*}, 10^{*}, 11^{*}, \\
12^{*}, 13^{*}, 14^{*}, 15^{*} \\
16^{*}, 17^{*}, 18^{*}, 19^{*} \\
20^{*}, 21^{*}, 22^{*}, 23^{*}\end{array}$ & \\
\hline 119 & $\begin{array}{c}\text { Linum strictum } \text { L./ } \\
\text { Linaceae } \\
\text { Alsi } \\
\text { SAN-SR-182 }\end{array}$ & $\begin{array}{l}\text { Herb/ } \\
\text { W }\end{array}$ & Leaves/ Paste & $\begin{array}{l}\text { Poultice, inflammation, } \\
\text { skin irritation }\end{array}$ & 3 & 0.25 & 12 & 0.03 & $\begin{array}{c}1^{*}, 2^{*}, 3^{*}, 4^{*}, 5^{*}, 6^{*}, \\
7^{*}, 8^{*}, 9^{*}, 10^{*}, 11^{*}, \\
12^{*}, 13^{*}, 14^{*}, 15^{*} \\
16^{*}, 17^{*}, 18^{*}, 19^{*} \\
20^{*}, 21^{*}, 22^{*}, 23^{*}\end{array}$ & \\
\hline
\end{tabular}

APPLIED ECOLOGY AND ENVIRONMENTAL RESEARCH 17(2):4725-4805.

http://www.aloki.hu • ISSN 15891623 (Print) • ISSN 17850037 (Online)

DOI: http://dx.doi.org/10.15666/aeer/1702_47254805

(c) 2019, ALÖKI Kft., Budapest, Hungary 


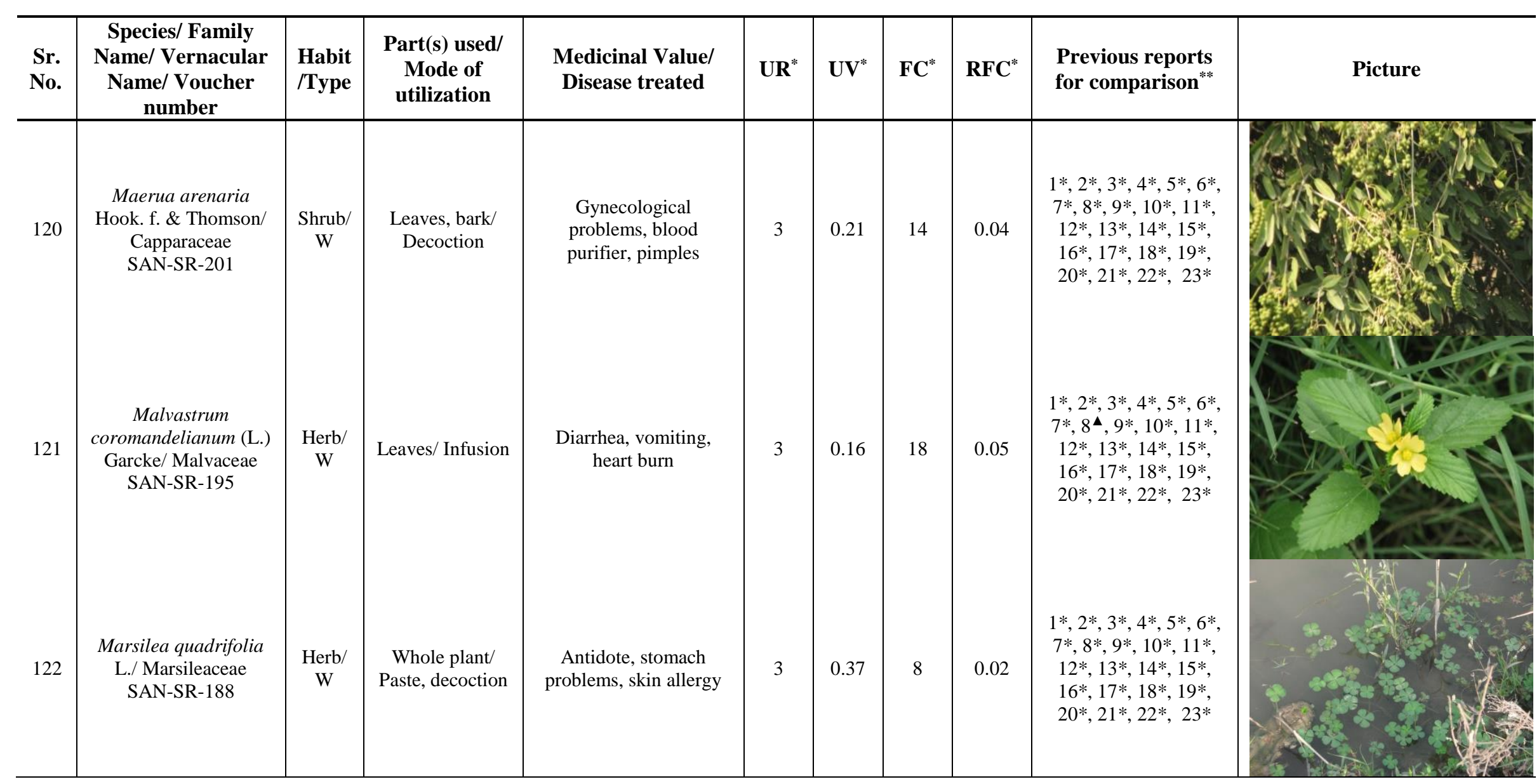




\begin{tabular}{|c|c|c|c|c|c|c|c|c|c|c|}
\hline $\begin{array}{l}\text { Sr. } \\
\text { No. }\end{array}$ & $\begin{array}{c}\text { Species/ Family } \\
\text { Name/ Vernacular } \\
\text { Name/ Voucher } \\
\text { number } \\
\end{array}$ & $\begin{array}{l}\text { Habit } \\
\text { /Type }\end{array}$ & $\begin{array}{l}\text { Part(s) used/ } \\
\text { Mode of } \\
\text { utilization }\end{array}$ & $\begin{array}{l}\text { Medicinal Value/ } \\
\text { Disease treated }\end{array}$ & $\mathbf{U} \mathbf{R}^{*}$ & $\mathbf{U V}^{*}$ & FC $^{*}$ & RFC $^{*}$ & $\begin{array}{l}\text { Previous reports } \\
\text { for comparison }\end{array}$ & Picture \\
\hline 123 & $\begin{array}{l}\text { Medicago sativa } \mathrm{L} \text {. } \\
\text { Fabaceae } \\
\text { Maina } \\
\text { SAN-SR-202 }\end{array}$ & $\begin{array}{l}\text { Herb/ } \\
\text { W }\end{array}$ & Flower/ Infusion & Hair tonic, & 1 & 0.33 & 3 & 0.01 & $\begin{array}{c}1^{*}, 2^{*}, 3^{*}, 4^{*}, 5^{*}, 6^{*} \\
7^{\star}, 8^{*}, 9^{*}, 10^{*}, 11^{*} \\
12^{*}, 13^{*}, 14^{*}, 15^{*} \\
16^{*}, 17^{*}, 18^{*}, 19^{*} \\
20^{*}, 21^{*}, 22^{*}, 23^{*}\end{array}$ & \\
\hline 124 & $\begin{array}{c}\text { Melilotus indicus (L.) } \\
\text { All./ Fabaceae } \\
\text { Maina } \\
\text { SAN-SR-216 }\end{array}$ & $\begin{array}{l}\text { Herb/ } \\
\text { W }\end{array}$ & $\begin{array}{l}\text { Whole plant/ } \\
\text { Juice }\end{array}$ & Wasp bite, inflammation & 2 & 0.12 & 16 & 0.04 & $\begin{array}{c}1^{*}, 2^{*}, 3^{*}, 4^{*}, 5^{*}, 6^{*}, \\
7^{*}, 8^{*}, 9^{*}, 10^{*}, 11^{*}, \\
12^{*}, 13^{*}, 14^{*}, 15^{*} \\
16^{*}, 17^{*}, 18^{*}, 19^{*} \\
2^{*}, 21^{*}, 22^{*}, 23^{*}\end{array}$ & \\
\hline 125 & $\begin{array}{c}\text { Mentha longifolia }(\mathrm{L} .) \\
\text { L./ Lamiaceae } \\
\text { Senji } \\
\text { SAN-SR-89 }\end{array}$ & $\begin{array}{l}\text { Herb/ } \\
\text { W }\end{array}$ & $\begin{array}{l}\text { Whole plant/ } \\
\text { Infusion, } \\
\text { decoction }\end{array}$ & $\begin{array}{c}\text { Gastrointestinal } \\
\text { disorders, obesity, } \\
\text { aphrodisiac, febrifuge, }\end{array}$ & 4 & 0.09 & 45 & 0.13 & $\begin{array}{c}1^{*}, 2^{\bullet}, 3^{*}, 4^{*}, 5^{*}, 6^{\bullet}, \\
7^{\bullet}, 8^{\bullet}, 9^{*}, 10^{*}, 11^{\bullet}, \\
12^{\bullet}, 13^{*}, 14^{*}, 15^{*}, \\
16^{*}, 17^{*}, 18^{*}, 19^{*}, \\
20^{*}, 21^{*}, 22^{*}, 23^{*}\end{array}$ & \\
\hline
\end{tabular}




\begin{tabular}{|c|c|c|c|c|c|c|c|c|c|c|}
\hline $\begin{array}{l}\text { Sr. } \\
\text { No. }\end{array}$ & $\begin{array}{c}\text { Species/ Family } \\
\text { Name/ Vernacular } \\
\text { Name/ Voucher } \\
\text { number } \\
\end{array}$ & $\begin{array}{l}\text { Habit } \\
\text { /Type }\end{array}$ & $\begin{array}{l}\text { Part(s) used/ } \\
\text { Mode of } \\
\text { utilization }\end{array}$ & $\begin{array}{l}\text { Medicinal Value/ } \\
\text { Disease treated }\end{array}$ & $\mathbf{U R}^{*}$ & $\mathbf{U V}^{*}$ & FC $^{*}$ & RFC $^{*}$ & $\begin{array}{l}\text { Previous reports } \\
\text { for comparison** }\end{array}$ & Picture \\
\hline 126 & $\begin{array}{c}\text { Merremia dissecta } \\
\text { (Jacq.) Hallier f./ } \\
\text { Convolvulaceae } \\
\text { SAN-SR-107 }\end{array}$ & $\begin{array}{l}\text { Herb/ } \\
\text { W }\end{array}$ & $\begin{array}{l}\text { Whole plant/ } \\
\text { Decoction }\end{array}$ & Cough, fever, headache & 3 & 0.6 & 5 & 0.01 & $\begin{array}{c}1^{*}, 2^{*}, 3^{*}, 4^{*}, 5^{*}, 6^{*}, \\
7^{*}, 8^{*}, 9^{*}, 10^{*}, 11^{*} \\
12^{*}, 13^{*}, 14^{*}, 15^{*} \\
16^{*}, 17^{*}, 18^{*}, 19^{*} \\
20^{*}, 21^{*}, 22^{*}, 23^{*}\end{array}$ & \\
\hline 127 & $\begin{array}{l}\text { Millettia pinnata } \\
\text { (L.) Panigrahi/ } \\
\text { Fabaceae } \\
\text { Sukh chain } \\
\text { SAN-SR-63 }\end{array}$ & $\begin{array}{l}\text { Tree/ } \\
\text { W,C }\end{array}$ & $\begin{array}{l}\text { Leaves/ } \\
\text { Decoction }\end{array}$ & $\begin{array}{c}\text { Febrifuge, respiratory } \\
\text { disorders, }\end{array}$ & 2 & 0.16 & 12 & 0.03 & $\begin{array}{c}1^{*}, 2^{*}, 3^{*}, 4^{*}, 5^{*}, 6^{*}, \\
7^{*}, 8^{*}, 9^{*}, 10^{*}, 11^{*}, \\
12^{*}, 13^{*}, 14^{*}, 15^{*} \\
16^{*}, 17^{*}, 18^{*}, 19^{*} \\
20^{*}, 21^{*}, 22^{*}, 23^{*}\end{array}$ & \\
\hline 128 & $\begin{array}{c}\text { Minuartia hybrida } \\
\text { (Vill.) Schischk./ } \\
\text { Caryophyllaceae } \\
\text { SAN-SR-41 }\end{array}$ & $\begin{array}{l}\text { Herb/ } \\
\text { W }\end{array}$ & ----- & ----- & 0 & 0 & 8 & 0.02 & $\begin{array}{c}1,2,3,4,5,6,7,8,9, \\
10,11,12,13,14,15, \\
16,17,18,19,20,21, \\
22,23\end{array}$ & \\
\hline 129 & $\begin{array}{c}\text { Misopates orontium } \\
\text { (L.) Raf./ } \\
\text { Plantaginaceae } \\
\text { SAN-SR-32 }\end{array}$ & $\begin{array}{l}\text { Herb/ } \\
\text { W }\end{array}$ & ----- & ----- & 0 & 0 & 6 & 0.02 & $\begin{array}{c}1,2,3,4,5,6,7,8,9, \\
10,11,12,13,14,15, \\
16,17,18,19,20,21, \\
22,23\end{array}$ & \\
\hline
\end{tabular}

APPLIED ECOLOGY AND ENVIRONMENTAL RESEARCH 17(2):4725-4805.

http://www.aloki.hu • ISSN 15891623 (Print) • ISSN 17850037 (Online)

DOI: http://dx.doi.org/10.15666/aeer/1702_47254805

(๖) 2019, ALÖKI Kft., Budapest, Hungary 


\begin{tabular}{|c|c|c|c|c|c|c|c|c|c|c|}
\hline $\begin{array}{l}\text { Sr. } \\
\text { No. }\end{array}$ & $\begin{array}{c}\text { Species/ Family } \\
\text { Name/ Vernacular } \\
\text { Name/ Voucher } \\
\text { number } \\
\end{array}$ & $\begin{array}{l}\text { Habit } \\
\text { /Type }\end{array}$ & $\begin{array}{l}\text { Part(s) used/ } \\
\text { Mode of } \\
\text { utilization }\end{array}$ & $\begin{array}{l}\text { Medicinal Value/ } \\
\text { Disease treated }\end{array}$ & $\mathbf{U} \mathbf{R}^{*}$ & $\mathbf{U V}^{*}$ & $\mathbf{F C}^{*}$ & RFC $^{*}$ & $\begin{array}{l}\text { Previous reports } \\
\text { for comparison }^{* *}\end{array}$ & Picture \\
\hline 130 & $\begin{array}{l}\text { Momordica balsamina } \\
\text { L./ Cucurbitaceae } \\
\text { Jangli karela } \\
\text { SAN-SR-37 }\end{array}$ & $\begin{array}{c}\text { Herb/ } \\
\text { W }\end{array}$ & Fruit/ Raw & $\begin{array}{l}\text { Diabetes, skin problems, } \\
\text { blood purifier, stomach } \\
\text { problems, throat } \\
\text { infection, liver problems }\end{array}$ & 6 & 0.24 & 25 & 0.07 & $\begin{array}{c}1^{*}, 2^{*}, 3^{*}, 4^{*}, 5^{*}, 6^{*}, \\
7^{*}, 8^{*}, 9^{*}, 10^{*}, 11^{*} \\
12^{*}, 13^{*}, 14^{*}, 15^{*} \\
16^{*}, 17^{*}, 18^{*}, 19^{*} \\
20^{*}, 21^{*}, 22^{*}, 23^{*}\end{array}$ & \\
\hline 131 & $\begin{array}{l}\text { Monotheca buxifolia } \\
\text { (Falc.) A. DC./ } \\
\text { Sapotaceae } \\
\text { Gurgura } \\
\text { SAN-SR-159 }\end{array}$ & $\begin{array}{c}\text { Tree/ } \\
\text { W }\end{array}$ & Fruit/ Raw & $\begin{array}{l}\text { Constipation, laxative, } \\
\text { cooling effect, liver } \\
\text { problems }\end{array}$ & 4 & 0.2 & 20 & 0.06 & $\begin{array}{c}1^{*}, 2^{*}, 3^{*}, 4^{*}, 5^{*}, 6^{*}, \\
7^{*}, 8^{*}, 9^{*}, 10^{*}, 11^{*} \\
12^{*}, 13^{*}, 14^{*}, 15^{*} \\
16^{*}, 17^{*}, 18^{*}, 19^{*} \\
20^{*}, 21^{*}, 22^{*}, 23^{*}\end{array}$ & \\
\hline 132 & $\begin{array}{c}\text { Moringa oleifera } \\
\text { Lam./ } \\
\text { Moringaceae/ } \\
\text { Sohanjna } \\
\text { SAN-SR-103 }\end{array}$ & $\begin{array}{l}\text { Tree/ } \\
\text { W,C }\end{array}$ & $\begin{array}{l}\text { Pods, leaves/ } \\
\text { Raw, powder }\end{array}$ & $\begin{array}{l}\text { Skin problems, wounds, } \\
\text { boils, depurative, } \\
\text { febrifuge, jaundice, } \\
\text { stomach problems }\end{array}$ & 7 & 0.23 & 30 & 0.08 & 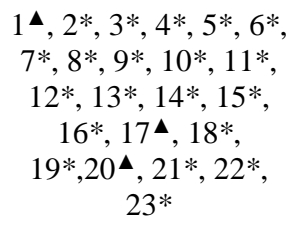 & \\
\hline
\end{tabular}




\begin{tabular}{|c|c|c|c|c|c|c|c|c|c|c|}
\hline $\begin{array}{l}\text { Sr. } \\
\text { No. }\end{array}$ & $\begin{array}{c}\text { Species/ Family } \\
\text { Name/ Vernacular } \\
\text { Name/ Voucher } \\
\text { number } \\
\end{array}$ & $\begin{array}{l}\text { Habit } \\
\text { /Type }\end{array}$ & $\begin{array}{l}\text { Part(s) used/ } \\
\text { Mode of } \\
\text { utilization }\end{array}$ & $\begin{array}{l}\text { Medicinal Value/ } \\
\text { Disease treated }\end{array}$ & $\mathbf{U R}^{*}$ & $\mathbf{U V}^{*}$ & $\mathbf{F C}^{*}$ & RFC $^{*}$ & $\begin{array}{l}\text { Previous reports } \\
\text { for comparison }\end{array}$ & Picture \\
\hline 133 & $\begin{array}{l}\text { Morus alba L./ } \\
\text { Moraceae } \\
\text { Safaid toot } \\
\text { SAN-SR-146 }\end{array}$ & $\begin{array}{c}\text { Tree/ } \\
\text { C }\end{array}$ & Fruit/ Raw & $\begin{array}{l}\text { Laxative, expectorant, } \\
\text { respiratory disorders, } \\
\text { jaundice }\end{array}$ & 4 & 0.12 & 33 & 0.09 & 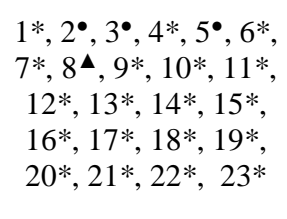 & \\
\hline 134 & $\begin{array}{c}\text { Morus laevigata Wall. } \\
\text { ex Brandis/ Moraceae } \\
\text { Shah toot } \\
\text { SAN-SR-149 }\end{array}$ & $\begin{array}{c}\text { Tree/ } \\
\text { C }\end{array}$ & Fruit/ Raw & $\begin{array}{l}\text { Laxative, expectorant, } \\
\text { respiratory disorders, } \\
\text { jaundice }\end{array}$ & 4 & 0.18 & 22 & 0.06 & $\begin{array}{c}1^{*}, 2^{*}, 3^{*}, 4^{*}, 5^{*}, 6^{*}, \\
7^{*}, 8^{*}, 9^{*}, 10^{*}, 11^{*} \\
12^{*}, 13^{*}, 14^{*}, 15^{*} \\
16^{*}, 17^{*}, 18^{*}, 19^{*} \\
20^{*}, 21^{*}, 22^{*}, 23^{*}\end{array}$ & \\
\hline 135 & $\begin{array}{c}\text { Morus nigra L./ } \\
\text { Moraceae } \\
\text { Kala toot } \\
\text { SAN-SR-209 }\end{array}$ & $\begin{array}{c}\text { Tree/ } \\
\text { C }\end{array}$ & Fruit/ Raw & $\begin{array}{l}\text { Laxative, expectorant, } \\
\text { respiratory disorders, } \\
\text { jaundice, }\end{array}$ & 4 & 0.13 & 29 & 0.08 & $\begin{array}{c}1^{*}, 2^{\bullet}, 3^{*}, 4^{*}, 5^{\bullet}, 6^{*}, \\
7^{*}, 8^{*}, 9^{*}, 10^{*}, 11^{*} \\
12^{*}, 13^{*}, 14^{*}, 15^{*} \\
16^{*}, 17^{*}, 18^{*}, 19^{*} \\
20^{*}, 21^{*}, 22^{*}, 23^{*}\end{array}$ & \\
\hline 136 & $\begin{array}{c}\text { Nannorrhops } \\
\text { ritchieana (Griff.) } \\
\text { Aitch./ Arecaceae } \\
\text { Mazri } \\
\text { SAN-SR-186 }\end{array}$ & $\begin{array}{c}\text { Shrub/ } \\
\text { W }\end{array}$ & $\begin{array}{c}\text { Fruit, leaves/ } \\
\text { Raw }\end{array}$ & Tonic & 1 & 0.2 & 5 & 0.01 & $\begin{array}{c}1^{*}, 2^{*}, 3^{*}, 4^{*}, 5^{*}, 6^{*} \\
7^{*}, 8^{*}, 9^{*}, 10^{*}, 11^{*} \\
12^{*}, 13^{*}, 14^{*}, 15^{*} \\
16^{*}, 17^{*}, 18^{*}, 19^{*} \\
20^{*}, 21^{*}, 22^{*}, 23^{*}\end{array}$ & \\
\hline
\end{tabular}

APPLIED ECOLOGY AND ENVIRONMENTAL RESEARCH 17(2):4725-4805.

http://www.aloki.hu • ISSN 15891623 (Print) • ISSN 17850037 (Online)

DOI: http://dx.doi.org/10.15666/aeer/1702_47254805

(c) 2019, ALÖKI Kft., Budapest, Hungary 


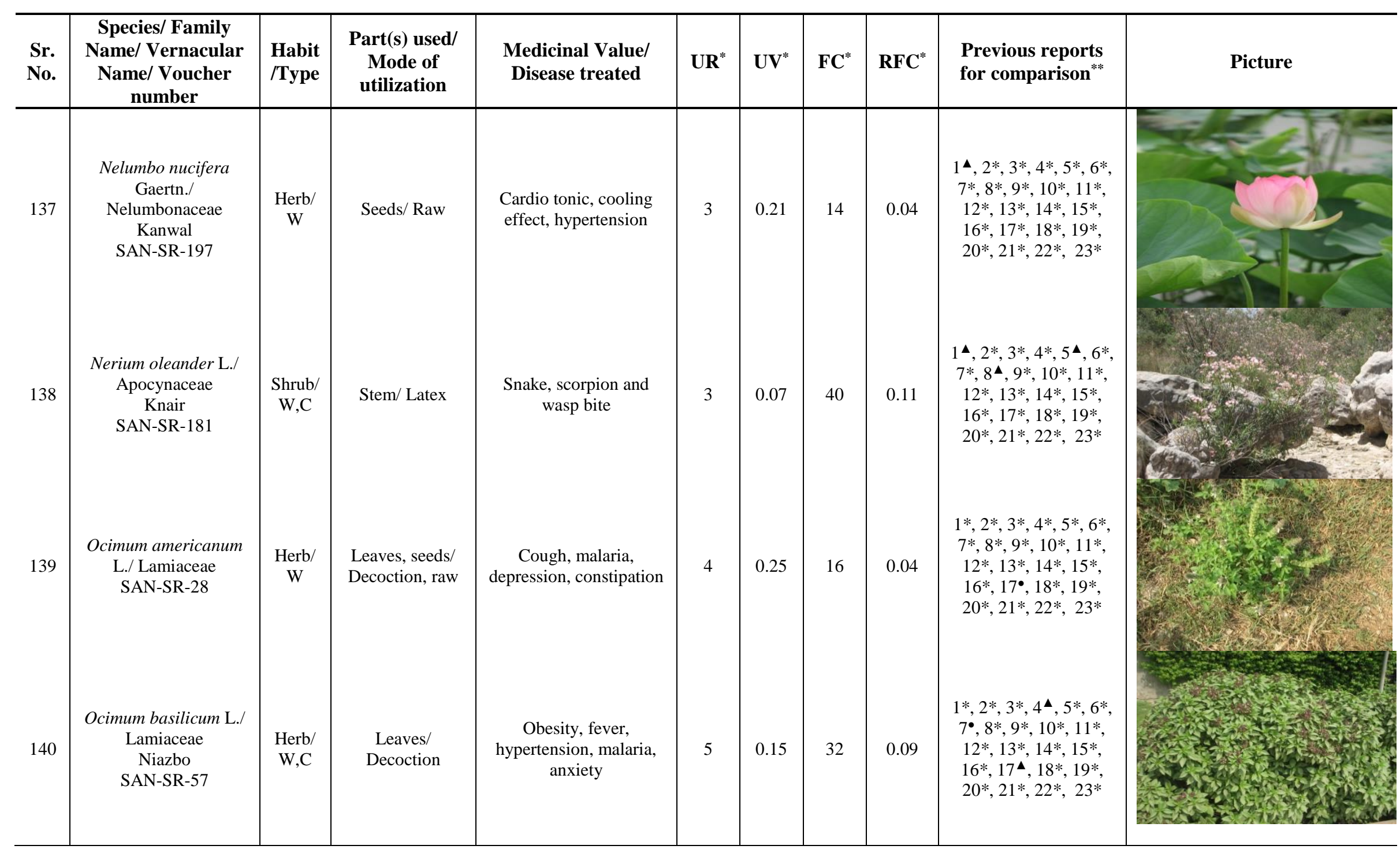

APPLIED ECOLOGY AND ENVIRONMENTAL RESEARCH 17(2):4725-4805.

http://www aloki.hu • ISSN 15891623 (Print) • ISSN 17850037 (Online)

DOI: http://dx.doi.org/10.15666/aeer/1702_47254805

(c) 2019, ALÖKI Kft., Budapest, Hungary 


\begin{tabular}{|c|c|c|c|c|c|c|c|c|c|c|}
\hline $\begin{array}{l}\text { Sr. } \\
\text { No. }\end{array}$ & $\begin{array}{c}\text { Species/ Family } \\
\text { Name/ Vernacular } \\
\text { Name/ Voucher } \\
\text { number } \\
\end{array}$ & $\begin{array}{l}\text { Habit } \\
\text { /Type }\end{array}$ & $\begin{array}{l}\text { Part(s) used/ } \\
\text { Mode of } \\
\text { utilization }\end{array}$ & $\begin{array}{l}\text { Medicinal Value/ } \\
\text { Disease treated }\end{array}$ & $\mathbf{U} \mathbf{R}^{*}$ & $\mathbf{U V}^{*}$ & $\mathbf{F C}^{*}$ & RFC $^{*}$ & $\begin{array}{l}\text { Previous reports } \\
\text { for comparison** }\end{array}$ & Picture \\
\hline 141 & $\begin{array}{l}\text { Olea ferruginea Wall. } \\
\text { ex Aitch./ Oleaceae } \\
\text { SAN-SR-111 }\end{array}$ & $\begin{array}{l}\text { Tree/ } \\
\mathrm{W}\end{array}$ & Fruit/ Oil & $\begin{array}{l}\text { Laxative, obesity, } \\
\text { stomach ache, tonic, } \\
\text { rheumatism }\end{array}$ & 6 & 0.22 & 27 & 0.08 & 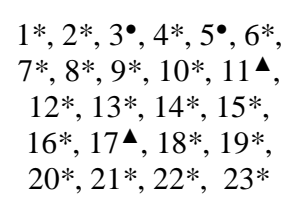 & \\
\hline 142 & $\begin{array}{c}\text { Opuntia dillenii (Ker } \\
\text { Gawl.) Haw./ } \\
\text { Cactaceae } \\
\text { SAN-SR-115 }\end{array}$ & $\begin{array}{l}\text { Shrub/ } \\
\text { W }\end{array}$ & $\begin{array}{l}\text { Fruit, leaves/ } \\
\text { Juice, extract }\end{array}$ & $\begin{array}{l}\text { Cooling effect, liver } \\
\text { problems, inflammation }\end{array}$ & 3 & 0.16 & 19 & 0.05 & 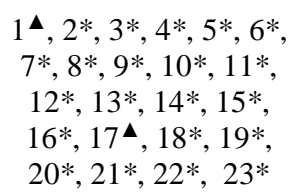 & \\
\hline 143 & $\begin{array}{l}\text { Otostegia limbata } \\
\text { (Benth.) Boiss/ } \\
\text { Lamiaceae } \\
\text { SAN-SR-205 }\end{array}$ & $\begin{array}{l}\text { Shrub/ } \\
\text { W }\end{array}$ & $\begin{array}{l}\text { Flowers, leaves/ } \\
\text { Decoction, } \\
\text { infusion }\end{array}$ & $\begin{array}{l}\text { Hypertension, insomnia, } \\
\text { cough, sore throat }\end{array}$ & 4 & 0.33 & 12 & 0.03 & 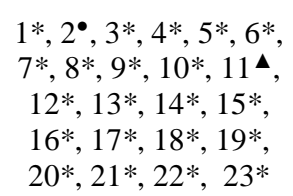 & \\
\hline
\end{tabular}




\begin{tabular}{|c|c|c|c|c|c|c|c|c|c|c|}
\hline $\begin{array}{l}\text { Sr. } \\
\text { No. }\end{array}$ & $\begin{array}{c}\text { Species/ Family } \\
\text { Name/ Vernacular } \\
\text { Name/ Voucher } \\
\text { number } \\
\end{array}$ & $\begin{array}{l}\text { Habit } \\
\text { /Type }\end{array}$ & $\begin{array}{l}\text { Part(s) used/ } \\
\text { Mode of } \\
\text { utilization }\end{array}$ & $\begin{array}{l}\text { Medicinal Value/ } \\
\text { Disease treated }\end{array}$ & UR ${ }^{*}$ & $\mathbf{U V} \mathbf{V}^{*}$ & $\mathbf{F C}^{*}$ & RFC $^{*}$ & $\begin{array}{l}\text { Previous reports } \\
\text { for comparison** }\end{array}$ & Picture \\
\hline 144 & $\begin{array}{c}\text { Oxalis corniculata } \text { L./ } \\
\text { Oxalidaceae } \\
\text { Khatti buti } \\
\text { SAN-SR-169 }\end{array}$ & $\begin{array}{l}\text { Herb/ } \\
\text { W }\end{array}$ & $\begin{array}{l}\text { Whole plant/ } \\
\text { Decoction }\end{array}$ & $\begin{array}{l}\text { Digestive problems, } \\
\text { nausea, vomiting, } \\
\text { bloating }\end{array}$ & 4 & 0.23 & 17 & 0.05 & $\begin{array}{c}1^{*}, 2^{\bullet}, 3^{*}, 4^{*}, 5^{*}, 6^{*}, \\
7^{*}, 8^{\mathbf{4}}, 9^{*}, 10^{*}, 11^{\bullet} \\
12^{*}, 13^{*}, 14^{*}, 15^{*} \\
16^{\bullet}, 17^{*}, 18^{*}, 19^{\bullet} \\
20^{\mathbf{4}}, 21^{*}, 22^{*}, 23^{*}\end{array}$ & \\
\hline 145 & $\begin{array}{c}\text { Oxalis corymbosa } \\
\text { DC./ Oxalidaceae } \\
\text { SAN-SR-173 }\end{array}$ & $\begin{array}{l}\text { Herb/ } \\
\text { W }\end{array}$ & $\begin{array}{l}\text { Whole plant/ } \\
\text { Decoction }\end{array}$ & $\begin{array}{l}\text { Digestive problems, } \\
\text { nausea, vomiting, } \\
\text { bloating }\end{array}$ & 4 & 0.23 & 17 & 0.05 & $\begin{array}{c}1^{*}, 2^{*}, 3^{*}, 4^{*}, 5^{*}, 6^{*}, \\
7^{*}, 8^{*}, 9^{*}, 10^{*}, 11^{*} \\
12^{*}, 13^{*}, 14^{*}, 15^{*} \\
16^{*}, 17^{*}, 18^{*}, 19^{*} \\
20^{*}, 21^{*}, 22^{*}, 23^{*}\end{array}$ & \\
\hline 146 & $\begin{array}{l}\text { Papaver dubium L./ } \\
\text { Papaveraceae } \\
\text { Jangli afyun } \\
\text { SAN-SR-14 }\end{array}$ & $\begin{array}{l}\text { Herb/ } \\
\mathrm{W}\end{array}$ & $\begin{array}{l}\text { Leaves, seeds/ } \\
\text { Infusion, juice }\end{array}$ & $\begin{array}{l}\text { Laxative, fatigue, } \\
\text { insomnia, cooling } \\
\text { effect, respiratory } \\
\text { problems, tonic }\end{array}$ & 6 & 0.26 & 23 & 0.06 & $\begin{array}{c}1^{*}, 2^{*}, 3^{*}, 4^{*}, 5^{*}, 6^{*} \\
7^{*}, 8^{*}, 9^{*}, 10^{*}, 11^{*} \\
12^{*}, 13^{*}, 14^{*}, 15^{*} \\
16^{*}, 17^{*}, 18^{*}, 19^{*} \\
20^{*}, 21^{*}, 22^{*}, 23^{*}\end{array}$ & \\
\hline 147 & $\begin{array}{c}\text { Parthenium } \\
\text { hysterophorus L./ } \\
\text { Asteraceae } \\
\text { Dhania booti } \\
\text { SAN-SR-39 }\end{array}$ & $\begin{array}{l}\text { Herb/ } \\
\text { W }\end{array}$ & $\begin{array}{l}\text { Whole plant/ } \\
\text { Paste, infusion } \\
\text { decoction }\end{array}$ & $\begin{array}{c}\text { Piles, poor appetite, } \\
\text { gynaecological } \\
\text { disorders }\end{array}$ & 3 & 0.18 & 16 & 0.04 & $\begin{array}{c}1^{*}, 2^{*}, 3^{*}, 4^{*}, 5^{*}, 6^{*}, \\
7^{*}, 8^{*}, 9^{*}, 10^{*}, 11^{*} \\
12^{*}, 13^{*}, 14^{*}, 15^{*} \\
16^{*}, 17^{*}, 18^{*}, 19^{*} \\
20^{*}, 21^{*}, 22^{*}, 23^{*}\end{array}$ & \\
\hline
\end{tabular}




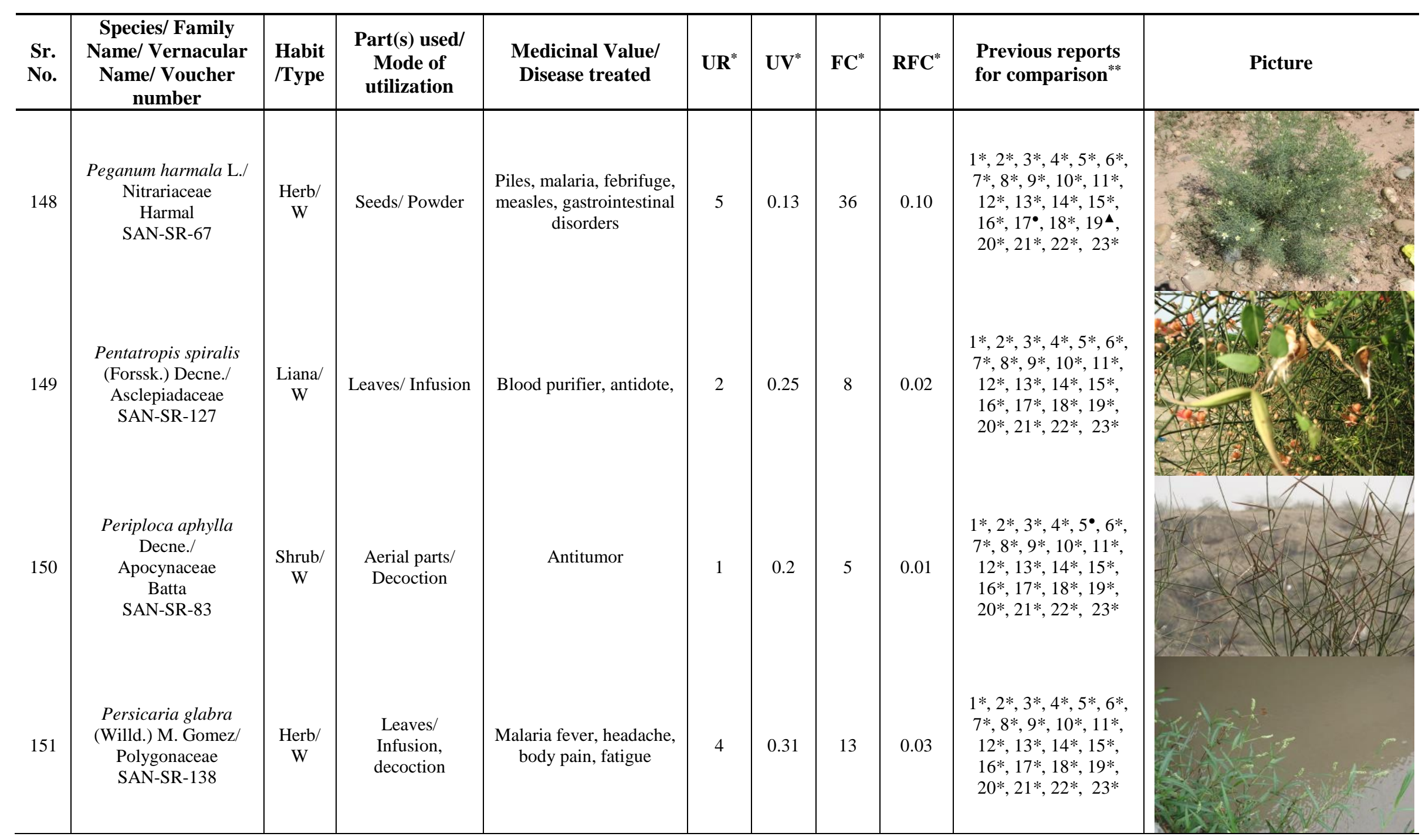

APPLIED ECOLOGY AND ENVIRONMENTAL RESEARCH 17(2):4725-4805.

http://www.aloki.hu • ISSN 15891623 (Print) • ISSN 17850037 (Online)

DOI: http://dx.doi.org/10.15666/aeer/1702_47254805

(c) 2019, ALÖKI Kft., Budapest, Hungary 


\begin{tabular}{|c|c|c|c|c|c|c|c|c|c|c|}
\hline $\begin{array}{l}\text { Sr. } \\
\text { No. }\end{array}$ & $\begin{array}{c}\text { Species/ Family } \\
\text { Name/ Vernacular } \\
\text { Name/ Voucher } \\
\text { number } \\
\end{array}$ & $\begin{array}{l}\text { Habit } \\
\text { /Type }\end{array}$ & $\begin{array}{l}\text { Part(s) used/ } \\
\text { Mode of } \\
\text { utilization }\end{array}$ & $\begin{array}{l}\text { Medicinal Value/ } \\
\text { Disease treated }\end{array}$ & $\mathbf{U R}^{*}$ & $\mathbf{U V}^{*}$ & $\mathbf{F C}^{*}$ & RFC $^{*}$ & $\begin{array}{l}\text { Previous reports } \\
\text { for comparison** }\end{array}$ & Picture \\
\hline 152 & $\begin{array}{l}\text { Persicaria lapathifolia } \\
\text { (L.) Delabre (Syn. } \\
\text { Polygonum } \\
\text { lapathifolium L./ } \\
\text { Polygonaceae } \\
\text { SAN-SR-123 }\end{array}$ & $\begin{array}{c}\text { Herb/ } \\
\text { W }\end{array}$ & $\begin{array}{l}\text { Leaves/ } \\
\text { Infusion, } \\
\text { decoction }\end{array}$ & $\begin{array}{c}\text { Malaria fever, headache, } \\
\text { body pain, fatigue }\end{array}$ & 4 & 0.28 & 14 & 0.04 & $\begin{array}{c}1^{*}, 2^{*}, 3^{*}, 4^{*}, 5^{*}, 6^{*}, \\
7^{*}, 8^{*}, 9^{*}, 10^{*}, 11^{*}, \\
12^{*}, 13^{*}, 14^{*}, 15^{*}, \\
16^{*}, 17^{*}, 18^{*}, 19^{*}, \\
20^{*}, 21^{*}, 22^{*}, 23^{*}\end{array}$ & \\
\hline 153 & $\begin{array}{c}\text { Phoenix sylvestris (L.) } \\
\text { Roxb. / } \\
\text { Arecaceae } \\
\text { Khajoor } \\
\text { SAN-SR-95 }\end{array}$ & $\begin{array}{c}\text { Tree/ } \\
\text { W }\end{array}$ & $\begin{array}{l}\text { Fruit/ Raw, } \\
\text { infusion }\end{array}$ & $\begin{array}{l}\text { aphrodisiac, tonic, } \\
\text { stomach problems, } \\
\text { expectorant, gonorrhea, }\end{array}$ & 5 & 0.22 & 22 & 0.06 & $\begin{array}{c}1^{*}, 2^{*}, 3^{*}, 4^{*}, 5^{*}, 6^{*}, \\
7^{*}, 8^{*}, 9^{*}, 10^{*}, 11^{*}, \\
12^{*}, 13^{*}, 14^{*}, 15^{*}, \\
16^{*}, 17^{*}, 18^{*}, 19^{*}, \\
20^{*}, 21^{*}, 22^{*}, 23^{*}\end{array}$ & \\
\hline 154 & $\begin{array}{l}\text { Phragmites australis } \\
\text { (Cav.) Trin. ex Steud./ } \\
\text { Poaceae } \\
\text { Narri } \\
\text { SAN-SR-35 }\end{array}$ & $\begin{array}{c}\text { Herb/ } \\
\text { W }\end{array}$ & ----- & ----- & 0 & 0 & 15 & 0.04 & $\begin{array}{c}1,2,3,4,5,6,7,8,9, \\
10,11,12,13,14,15, \\
16,17,18,19,20,21, \\
22,23\end{array}$ & \\
\hline
\end{tabular}




\begin{tabular}{|c|c|c|c|c|c|c|c|c|c|c|}
\hline $\begin{array}{l}\text { Sr. } \\
\text { No. }\end{array}$ & $\begin{array}{c}\text { Species/ Family } \\
\text { Name/ Vernacular } \\
\text { Name/ Voucher } \\
\text { number } \\
\end{array}$ & $\begin{array}{l}\text { Habit } \\
\text { /Type }\end{array}$ & $\begin{array}{c}\text { Part(s) used/ } \\
\text { Mode of } \\
\text { utilization }\end{array}$ & $\begin{array}{l}\text { Medicinal Value/ } \\
\text { Disease treated }\end{array}$ & $\mathbf{U} \mathbf{R}^{*}$ & $\mathbf{U V}^{*}$ & $\mathbf{F C}^{*}$ & RFC $^{*}$ & $\begin{array}{l}\text { Previous reports } \\
\text { for comparison** }\end{array}$ & Picture \\
\hline 155 & $\begin{array}{c}\text { Phyla nodiflora }(\mathrm{L} .) \\
\text { Greene/ Verbenaceae } \\
\text { Bhukkan } \\
\text { SAN-SR-84 }\end{array}$ & $\begin{array}{l}\text { Herb/ } \\
\text { W }\end{array}$ & $\begin{array}{l}\text { Whole plant/ } \\
\text { Decoction }\end{array}$ & $\begin{array}{l}\text { Constipation, } \\
\text { respiratory problems, } \\
\text { skin problems, }\end{array}$ & 3 & 0.3 & 10 & 0.03 & $\begin{array}{c}1^{*}, 2^{*}, 3^{*}, 4^{*}, 5^{*}, 6^{*}, \\
7^{*}, 8^{*}, 9^{*}, 10^{*}, 11^{*} \\
12^{*}, 13^{*}, 14^{*}, 15^{*} \\
16^{*}, 17^{*}, 18^{*}, 19^{*} \\
20^{*}, 21^{*}, 22^{*}, 23^{*}\end{array}$ & \\
\hline 156 & $\begin{array}{l}\text { Physalis minima L./ } \\
\text { Solanaceae } \\
\text { Jangli cherry } \\
\text { SAN-SR-12 }\end{array}$ & $\begin{array}{l}\text { Herb/ } \\
\text { W }\end{array}$ & $\begin{array}{l}\text { Whole plant/ } \\
\text { Powder, paste, } \\
\text { infusion }\end{array}$ & Chest infections, cough & 2 & 0.25 & 8 & 0.02 & $\begin{array}{c}1^{*}, 2^{*}, 3^{*}, 4^{*}, 5^{*}, 6^{*}, \\
7^{*}, 8^{*}, 9^{*}, 10^{*}, 11^{*} \\
12^{*}, 13^{*}, 14^{*}, 15^{*} \\
16^{*}, 17^{\star}, 18^{*}, 19^{*} \\
20^{*}, 21^{*}, 22^{*}, 23^{*}\end{array}$ & \\
\hline 157 & $\begin{array}{l}\text { Physorrhynchus } \\
\text { chamaerapistrum } \\
\text { (Boiss.) Boiss./ } \\
\text { Brassicaceae } \\
\text { SAN-SR-19 }\end{array}$ & $\begin{array}{l}\text { Herb/ } \\
\text { W }\end{array}$ & $\begin{array}{l}\text { Whole plant/ } \\
\text { Infusion, paste }\end{array}$ & $\begin{array}{l}\text { Skin problems, } \\
\text { headache }\end{array}$ & 2 & 0.16 & 12 & 0.03 & $\begin{array}{c}1^{*}, 2^{*}, 3^{*}, 4^{*}, 5^{*}, 6^{*}, \\
7^{*}, 8^{*}, 9^{*}, 10^{*}, 11^{*}, \\
12^{*}, 13^{*}, 14^{*}, 15^{*} \\
16^{*}, 17^{*}, 18^{*}, 19^{*} \\
20^{*}, 21^{*}, 22^{*}, 23^{*}\end{array}$ & \\
\hline
\end{tabular}




\begin{tabular}{|c|c|c|c|c|c|c|c|c|c|c|}
\hline $\begin{array}{l}\text { Sr. } \\
\text { No. }\end{array}$ & $\begin{array}{c}\text { Species/ Family } \\
\text { Name/ Vernacular } \\
\text { Name/ Voucher } \\
\text { number } \\
\end{array}$ & $\begin{array}{l}\text { Habit } \\
\text { /Type }\end{array}$ & $\begin{array}{l}\text { Part(s) used/ } \\
\text { Mode of } \\
\text { utilization }\end{array}$ & $\begin{array}{l}\text { Medicinal Value/ } \\
\text { Disease treated }\end{array}$ & $\mathbf{U R}^{*}$ & $\mathbf{U V}^{*}$ & $\mathbf{F C}^{*}$ & RFC $^{*}$ & $\begin{array}{l}\text { Previous reports } \\
\text { for comparison** }\end{array}$ & Picture \\
\hline 158 & $\begin{array}{c}\text { Pistia stratiotes L./ } \\
\text { Araceae } \\
\text { SAN-SR-208 }\end{array}$ & $\begin{array}{c}\text { Herb/ } \\
\text { W }\end{array}$ & $\begin{array}{l}\text { Leaves/ } \\
\text { Decoction }\end{array}$ & $\begin{array}{l}\text { Malaria, fever, } \\
\text { headache, sore throat }\end{array}$ & 4 & 0.30 & 13 & 0.04 & $\begin{array}{c}1^{*}, 2^{*}, 3^{*}, 4^{*}, 5^{*}, 6^{*} \\
7^{*}, 8^{*}, 9^{*}, 10^{*}, 11^{*} \\
12^{*}, 13^{*}, 14^{*}, 15^{*} \\
16^{*}, 17^{*}, 18^{*}, 19^{*} \\
20^{*}, 21^{*}, 22^{*}, 23^{*}\end{array}$ & \\
\hline 159 & $\begin{array}{c}\text { Plantago boissieri } \\
\text { Hausskn. \& Bornm.L./ } \\
\text { Plantaginaceae } \\
\text { Jangli isabgol } \\
\text { SAN-SR-179 }\end{array}$ & $\begin{array}{l}\text { Herb/ } \\
\text { W }\end{array}$ & Husk & $\begin{array}{l}\text { Digestive problems, } \\
\text { cooling effect, obesity, } \\
\text { poor appetite }\end{array}$ & 4 & 0.33 & 12 & 0.03 & $\begin{array}{c}1^{*}, 2^{*}, 3^{*}, 4^{*}, 5^{*}, 6^{*}, \\
7^{*}, 8^{*}, 9^{*}, 10^{*}, 11^{*}, \\
12^{*}, 13^{*}, 14^{*}, 15^{*}, \\
16^{*}, 17^{*}, 18^{*}, 19^{*}, \\
20^{*}, 21^{*}, 22^{*}, 23^{*}\end{array}$ & \\
\hline 160 & $\begin{array}{c}\text { Pluchea arabica } \\
\text { (Boiss.) Qaiser \& } \\
\text { Lack/ Asteraceae } \\
\text { SAN-SR-86 }\end{array}$ & $\begin{array}{l}\text { Herb/ } \\
\text { W }\end{array}$ & $\begin{array}{l}\text { Whole plant/ } \\
\text { Decoction }\end{array}$ & $\begin{array}{l}\text { Obesity, stomach ache, } \\
\text { cough, fever }\end{array}$ & 4 & 0.44 & 9 & 0.02 & $\begin{array}{c}1^{*}, 2^{*}, 3^{*}, 4^{*}, 5^{*}, 6^{*}, \\
7^{*}, 8^{*}, 9^{*}, 10^{*}, 11^{*}, \\
12^{*}, 13^{*}, 14^{*}, 15^{*}, \\
16^{*}, 17^{*}, 18^{*}, 19^{*} \\
20^{*}, 21^{*}, 22^{*}, 23^{*}\end{array}$ & \\
\hline
\end{tabular}

APPLIED ECOLOGY AND ENVIRONMENTAL RESEARCH 17(2):4725-4805.

http://www.aloki.hu • ISSN 15891623 (Print) • ISSN 17850037 (Online)

DOI: http://dx.doi.org/10.15666/aeer/1702_47254805

(c) 2019, ALÖKI Kft., Budapest, Hungary 


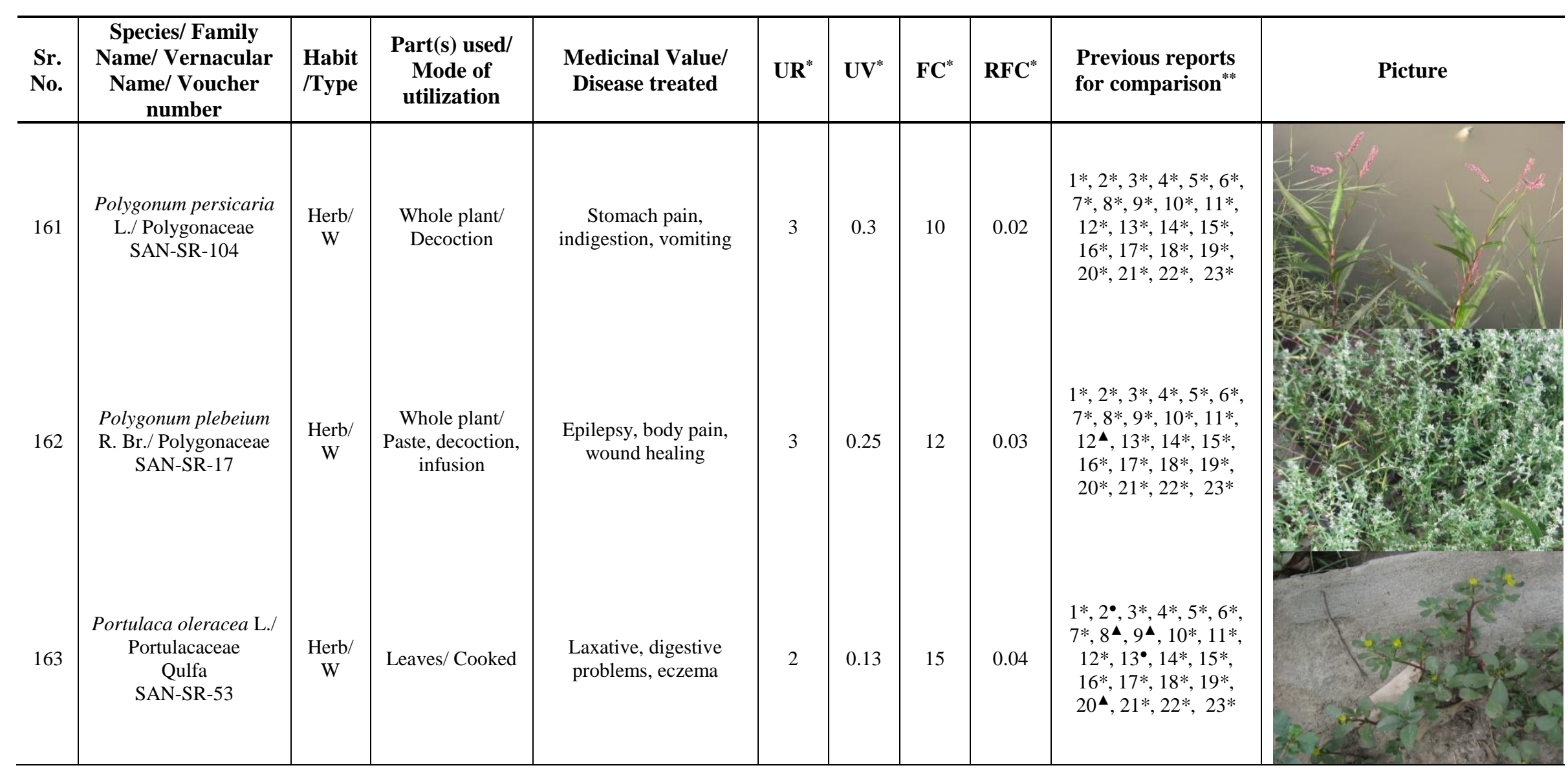




\begin{tabular}{|c|c|c|c|c|c|c|c|c|c|c|}
\hline $\begin{array}{l}\text { Sr. } \\
\text { No. }\end{array}$ & $\begin{array}{c}\text { Species/ Family } \\
\text { Name/ Vernacular } \\
\text { Name/ Voucher } \\
\text { number } \\
\end{array}$ & $\begin{array}{l}\text { Habit } \\
\text { /Type }\end{array}$ & $\begin{array}{l}\text { Part(s) used/ } \\
\text { Mode of } \\
\text { utilization }\end{array}$ & $\begin{array}{l}\text { Medicinal Value/ } \\
\text { Disease treated }\end{array}$ & $\mathbf{U} \mathbf{R}^{*}$ & $\mathbf{U V}^{*}$ & FC $^{*}$ & RFC $^{*}$ & $\begin{array}{l}\text { Previous reports } \\
\text { for comparison** }\end{array}$ & Picture \\
\hline 164 & $\begin{array}{c}\text { Prosopis } \\
\text { glandulosa Torr./ } \\
\text { Fabaceae } \\
\text { Angrezi kikri } \\
\text { SAN-SR-90 }\end{array}$ & $\begin{array}{l}\text { Shrub/ } \\
\text { W }\end{array}$ & Pods/ Decoction & $\begin{array}{l}\text { Gynaecological } \\
\text { disorders }\end{array}$ & 1 & 0.25 & 4 & 0.01 & $\begin{array}{c}1^{*}, 2^{*}, 3^{*}, 4^{*}, 5^{*}, 6^{*}, \\
7^{*}, 8^{*}, 9^{*}, 10^{*}, 11^{*} \\
12^{*}, 13^{*}, 14^{*}, 15^{*} \\
16^{*}, 17^{*}, 18^{*}, 19^{*} \\
20^{*}, 21^{*}, 22^{*}, 23^{*}\end{array}$ & \\
\hline 165 & $\begin{array}{c}\text { Prosopis cineraria }(\mathrm{L} .) \\
\text { Druce/ Fabaceae } \\
\text { Jand, Jandi } \\
\text { SAN-SR-119 }\end{array}$ & $\begin{array}{c}\text { Tree/ } \\
\mathrm{W}\end{array}$ & Leaves/ Smoke & $\begin{array}{l}\text { ENT problems, } \\
\text { inflammation, }\end{array}$ & 2 & 0.16 & 12 & 0.03 & $\begin{array}{c}1^{*}, 2^{*}, 3^{*}, 4^{*}, 5^{*}, 6^{*}, \\
7^{*}, 8^{*}, 9^{*}, 10^{*}, 11^{*}, \\
12^{*}, 13^{*}, 14^{*}, 15^{*} \\
16^{*}, 17^{*}, 18^{*}, 19^{*} \\
20^{*}, 21^{*}, 22^{*}, 23^{*}\end{array}$ & \\
\hline 166 & $\begin{array}{c}\text { Prosopis juliflora } \\
\text { (Sw.) DC./ } \\
\text { Fabaceae } \\
\text { Kikri } \\
\text { SAN-SR-18 }\end{array}$ & $\begin{array}{l}\text { Shrub/ } \\
\text { W }\end{array}$ & $\begin{array}{l}\text { Leaves, pods/ } \\
\text { Decoction }\end{array}$ & $\begin{array}{l}\text { Poultice, gynaecological } \\
\text { disorders }\end{array}$ & 2 & 0.08 & 23 & 0.06 & $\begin{array}{c}1^{*}, 2^{*}, 3^{*}, 4^{*}, 5^{*}, 6^{*} \\
7^{*}, 8^{*}, 9^{*}, 10^{*}, 11^{*} \\
12^{*}, 13^{*}, 14^{*}, 15^{*} \\
16^{*}, 17^{*}, 18^{*}, 19^{*} \\
20^{*}, 21^{*}, 22^{*}, 23^{*}\end{array}$ & \\
\hline 167 & $\begin{array}{c}\text { Pseudogaillonia } \\
\text { hymenostephana } \\
\text { (Jaub. \& Spach) } \\
\text { Linchevskii/ } \\
\text { Rubiaceae } \\
\text { SAN-SR-49 }\end{array}$ & $\begin{array}{l}\text { Herb/ } \\
\text { W }\end{array}$ & $\begin{array}{l}\text { Whole plant/ } \\
\text { Decoction }\end{array}$ & $\begin{array}{l}\text { Malaria fever, fatigue, } \\
\text { body pain, cough, cold }\end{array}$ & 5 & 0.26 & 19 & 0.05 & $\begin{array}{c}1^{*}, 2^{*}, 3^{*}, 4^{*}, 5^{*}, 6^{*}, \\
7^{*}, 8^{*}, 9^{*}, 10^{*}, 11^{*} \\
12^{*}, 13^{*}, 14^{*}, 15^{*} \\
16^{*}, 17^{*}, 18^{*}, 19^{*} \\
20^{*}, 21^{*}, 22^{*}, 23^{*}\end{array}$ & \\
\hline
\end{tabular}

APPLIED ECOLOGY AND ENVIRONMENTAL RESEARCH 17(2):4725-4805.

http://www.aloki.hu • ISSN 15891623 (Print) • ISSN 17850037 (Online)

DOI: http://dx.doi.org/10.15666/aeer/1702_47254805

(c) 2019, ALÖKI Kft., Budapest, Hungary 


\begin{tabular}{|c|c|c|c|c|c|c|c|c|c|c|}
\hline $\begin{array}{l}\text { Sr. } \\
\text { No. }\end{array}$ & $\begin{array}{c}\text { Species/ Family } \\
\text { Name/ Vernacular } \\
\text { Name/ Voucher } \\
\text { number } \\
\end{array}$ & $\begin{array}{l}\text { Habit } \\
\text { /Type }\end{array}$ & $\begin{array}{l}\text { Part(s) used/ } \\
\text { Mode of } \\
\text { utilization }\end{array}$ & $\begin{array}{l}\text { Medicinal Value/ } \\
\text { Disease treated }\end{array}$ & $\mathbf{U R}^{*}$ & $\mathbf{U} \mathbf{V}^{*}$ & $\mathbf{F C}^{*}$ & RFC $^{*}$ & $\begin{array}{l}\text { Previous reports } \\
\text { for comparison }{ }^{* *}\end{array}$ & Picture \\
\hline 168 & $\begin{array}{c}\text { Pulicaria glutinosa } \\
\text { (Boiss.) Jaub. \& } \\
\text { Spach/ } \\
\text { Asteraceae } \\
\text { SAN-SR-31 }\end{array}$ & $\begin{array}{l}\text { Herb/ } \\
\text { W }\end{array}$ & $\begin{array}{l}\text { Whole plant/ } \\
\text { Smoke, infusion }\end{array}$ & $\begin{array}{l}\text { Evil eye, measles, skin } \\
\text { allergy, inflammation, } \\
\text { malaria, blood purifier }\end{array}$ & 6 & 0.35 & 17 & 0.05 & $\begin{array}{c}1^{*}, 2^{*}, 3^{*}, 4^{*}, 5^{*}, 6^{*}, \\
7^{*}, 8^{*}, 9^{*}, 10^{*}, 11^{*} \\
12^{*}, 13^{*}, 14^{*}, 15^{*} \\
16^{*}, 17^{*}, 18^{*}, 19^{*} \\
20^{*}, 21^{*}, 22^{*}, 23^{*}\end{array}$ & \\
\hline 169 & $\begin{array}{c}\text { Punica granatum L./ } \\
\text { Lythraceae } \\
\text { SAN-SR-71 }\end{array}$ & $\begin{array}{l}\text { Shrub/ } \\
\text { W }\end{array}$ & $\begin{array}{l}\text { Fruit, peel/ } \\
\text { Juice, paste }\end{array}$ & $\begin{array}{l}\text { Malaria, pimples, } \\
\text { anemia, cooling effect, } \\
\text { fatigue, weakness }\end{array}$ & 6 & 0.24 & 25 & 0.07 & 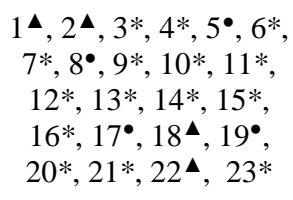 & \\
\hline 170 & $\begin{array}{c}\text { Pupalia lappacea (L.) } \\
\text { Juss./ Amaranthaceae } \\
\text { Gol Puthkanda } \\
\text { SAN-SR-76 }\end{array}$ & $\begin{array}{l}\text { Herb/ } \\
\text { W }\end{array}$ & Leaves/ Paste & Wound healing & 1 & 0.05 & 21 & 0.06 & $\begin{array}{c}1^{*}, 2^{*}, 3^{*}, 4^{*}, 5^{*}, 6^{*}, \\
7^{*}, 8^{*}, 9^{*}, 10^{*}, 11^{*}, \\
12^{*}, 13^{*}, 14^{*}, 15^{*} \\
16^{*}, 17^{\star}, 18^{*}, 19^{*} \\
20^{*}, 21^{*}, 22^{*}, 2^{*}\end{array}$ & \\
\hline
\end{tabular}




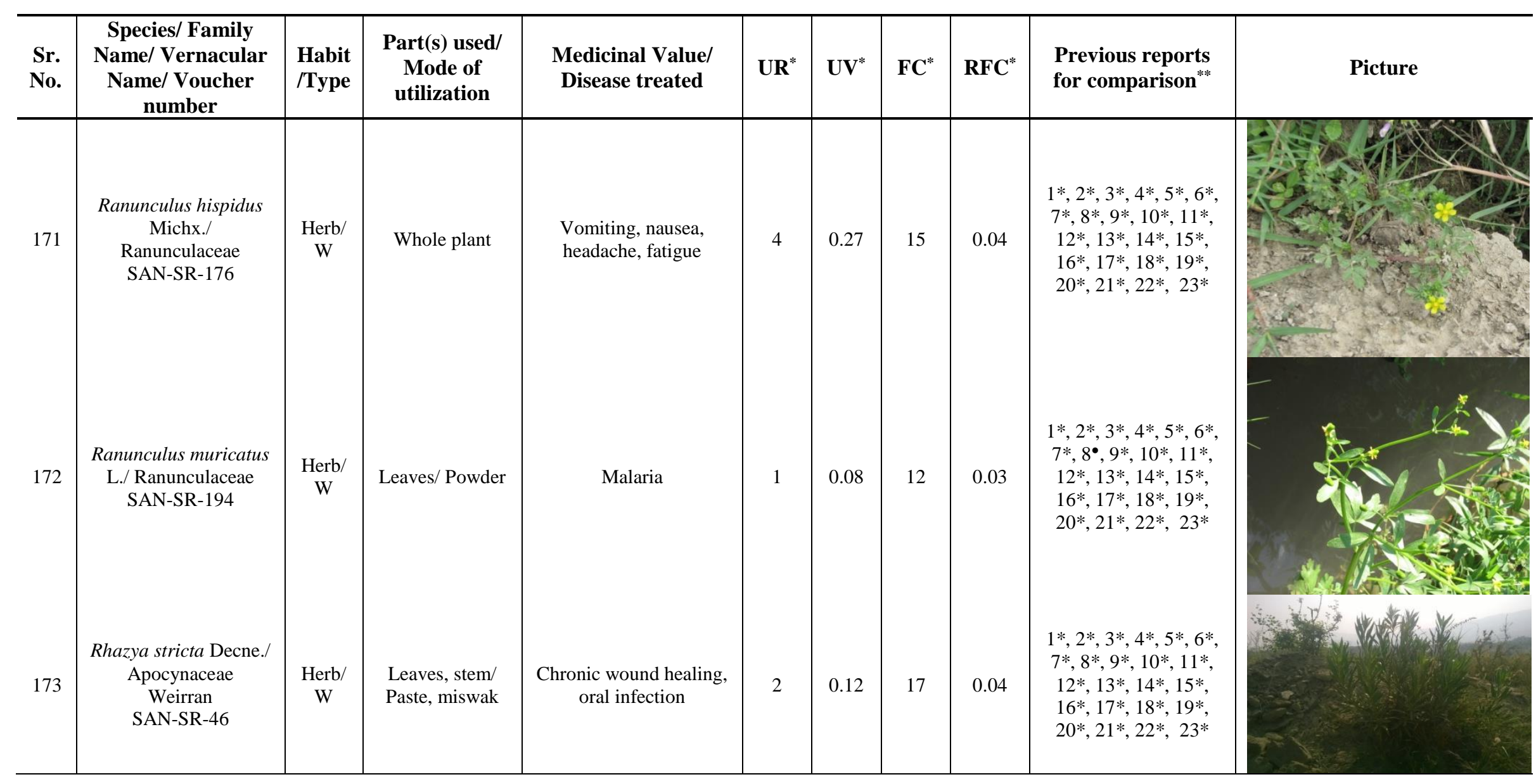




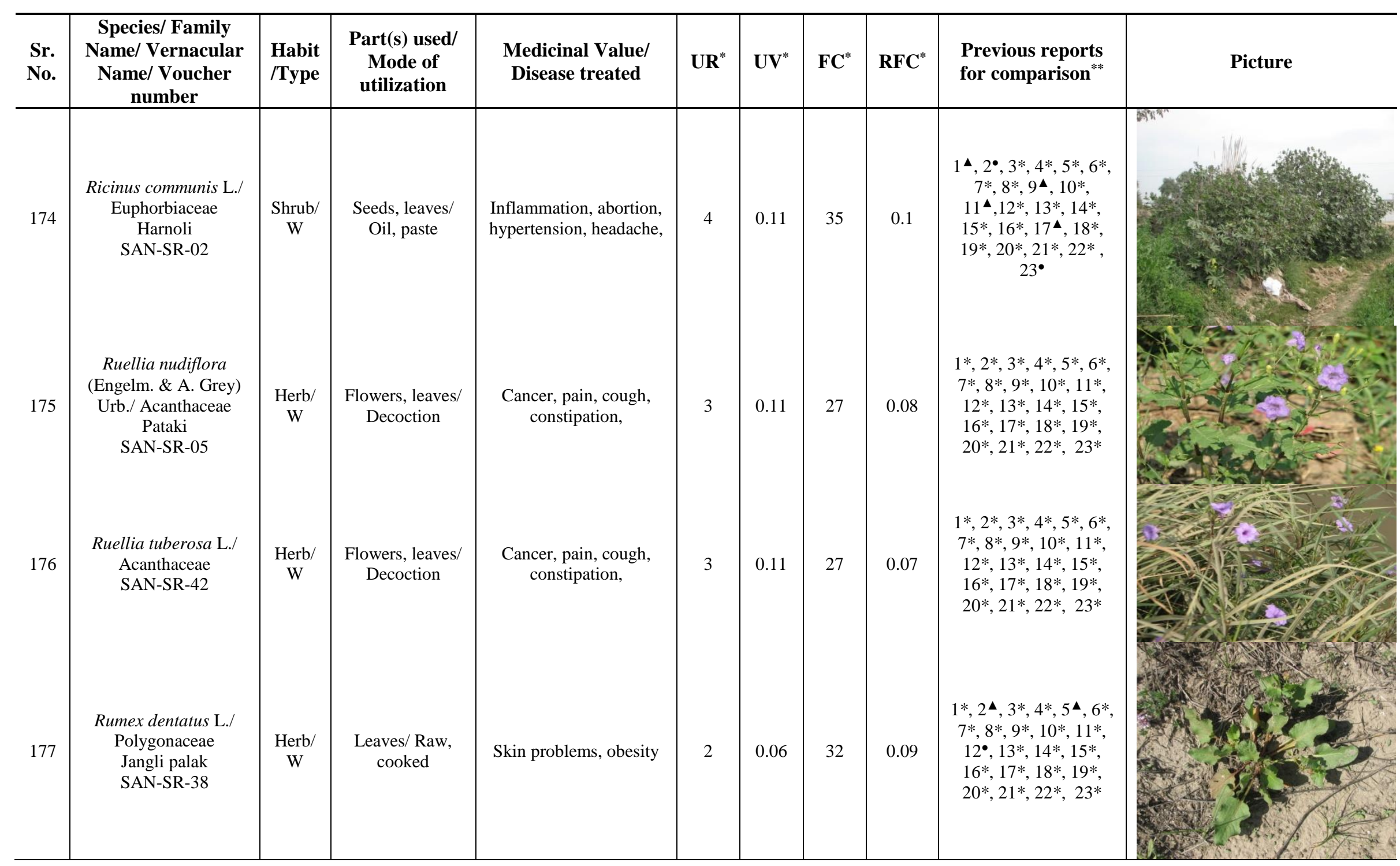

APPLIED ECOLOGY AND ENVIRONMENTAL RESEARCH 17(2):4725-4805.

http://www.aloki.hu • ISSN 15891623 (Print) • ISSN 17850037 (Online)

DOI: http://dx.doi.org/10.15666/aeer/1702_47254805

(๖) 2019, ALÖKI Kft., Budapest, Hungary 


\begin{tabular}{|c|c|c|c|c|c|c|c|c|c|c|}
\hline $\begin{array}{l}\text { Sr. } \\
\text { No. }\end{array}$ & $\begin{array}{c}\text { Species/ Family } \\
\text { Name/ Vernacular } \\
\text { Name/ Voucher } \\
\text { number } \\
\end{array}$ & $\begin{array}{l}\text { Habit } \\
\text { /Type }\end{array}$ & $\begin{array}{l}\text { Part(s) used/ } \\
\text { Mode of } \\
\text { utilization }\end{array}$ & $\begin{array}{l}\text { Medicinal Value/ } \\
\text { Disease treated }\end{array}$ & UR ${ }^{*}$ & $\mathbf{U V}^{*}$ & $\mathbf{F C}^{*}$ & RFC $^{*}$ & $\begin{array}{l}\text { Previous reports } \\
\text { for comparison** }\end{array}$ & Picture \\
\hline 178 & $\begin{array}{l}\text { Rumex vesicarius L./ } \\
\text { Polygonaceae } \\
\text { SAN-SR-70 }\end{array}$ & $\begin{array}{l}\text { Herb/ } \\
\text { W }\end{array}$ & $\begin{array}{c}\text { Leaves/ } \\
\text { Decoction, paste }\end{array}$ & $\begin{array}{l}\text { ENT problems, scorpion } \\
\text { bite, wasp bite, } \\
\text { indigestion, }\end{array}$ & 4 & 0.15 & 26 & 0.07 & $\begin{array}{c}1^{*}, 2^{*}, 3^{*}, 4^{*}, 5^{*}, 6^{*}, \\
7^{*}, 8^{*}, 9^{*}, 10^{*}, 11^{*} \\
12^{*}, 13^{*}, 14^{*}, 15^{*} \\
16^{*}, 17^{*}, 18^{*}, 19^{*} \\
20^{*}, 21^{*}, 22^{*}, 23^{*}\end{array}$ & \\
\hline 179 & $\begin{array}{c}\text { Saccharum bengalense } \\
\text { Retz./ Poaceae } \\
\text { Kana } \\
\text { SAN-SR-98 }\end{array}$ & $\begin{array}{l}\text { Herb/ } \\
\text { W }\end{array}$ & ----- & ----- & 0 & 0 & 19 & 0.05 & $\begin{array}{c}1,2,3,4,5,6,7,8,9, \\
10,11,12,13,14,15, \\
16,17,18,19,20,21, \\
22,23\end{array}$ & \\
\hline 180 & $\begin{array}{l}\text { Saccharum revennae } \\
\text { (L.) L./ Poaceae } \\
\text { Sarkanda } \\
\text { SAN-SR-62 }\end{array}$ & $\begin{array}{c}\text { Herb/ } \\
\text { W }\end{array}$ & ----- & ----- & 0 & 0 & 13 & 0.03 & $\begin{array}{c}1,2,3,4,5,6,7,8,9, \\
10,11,12,13,14,15, \\
16,17,18,19,20,21, \\
22,23\end{array}$ & \\
\hline
\end{tabular}




\begin{tabular}{|c|c|c|c|c|c|c|c|c|c|c|}
\hline $\begin{array}{l}\text { Sr. } \\
\text { No. }\end{array}$ & $\begin{array}{c}\text { Species/ Family } \\
\text { Name/ Vernacular } \\
\text { Name/ Voucher } \\
\text { number } \\
\end{array}$ & $\begin{array}{l}\text { Habit } \\
\text { /Type }\end{array}$ & $\begin{array}{l}\text { Part(s) used/ } \\
\text { Mode of } \\
\text { utilization }\end{array}$ & $\begin{array}{l}\text { Medicinal Value/ } \\
\text { Disease treated }\end{array}$ & $\mathbf{U R}^{*}$ & $\mathbf{U V}^{*}$ & FC $^{*}$ & RFC $^{*}$ & $\begin{array}{l}\text { Previous reports } \\
\text { for comparison** }\end{array}$ & Picture \\
\hline 181 & $\begin{array}{c}\text { Saccharum } \\
\text { spontaneum L./ } \\
\text { Poaceae } \\
\text { Kahn } \\
\text { SAN-SR-72 }\end{array}$ & $\begin{array}{l}\text { Herb/ } \\
\text { W }\end{array}$ & ----- & ----- & 0 & 0 & 20 & 0.05 & $\begin{array}{c}1,2,3,4,5,6,7,8,9, \\
10,11,12,13,14,15, \\
16,17,18,19,20,21, \\
22,23\end{array}$ & \\
\hline 182 & $\begin{array}{c}\text { Salvadora persica L./ } \\
\text { Salvadoraceae } \\
\text { Jaal, peelu } \\
\text { SAN-SR-88 }\end{array}$ & $\begin{array}{l}\text { Tree/ } \\
\text { W }\end{array}$ & $\begin{array}{c}\text { Fruits, bark/ } \\
\text { Raw, Decoction }\end{array}$ & $\begin{array}{c}\text { Toothache, epilepsy, } \\
\text { abortion }\end{array}$ & 3 & 0.07 & 40 & 0.11 & $\begin{array}{c}1^{*}, 2^{*}, 3^{*}, 4^{*}, 5^{*}, 6^{*}, \\
7^{*}, 8^{*}, 9^{*}, 10^{*}, 11^{*}, \\
12^{*}, 13^{*}, 14^{\star}, 15^{*}, \\
16^{*}, 17^{\star}, 18^{*}, 19^{*} \\
20^{*}, 21^{*}, 22^{*}, 23^{\mathbf{\Lambda}}\end{array}$ & \\
\hline 183 & $\begin{array}{c}\text { Salvia moorcroftiana } \\
\text { Wall. ex Benth./ } \\
\text { Lamiaceae } \\
\text { SAN-SR-99 }\end{array}$ & $\begin{array}{l}\text { Herb/ } \\
\text { W }\end{array}$ & $\begin{array}{l}\text { Leaves/ } \\
\text { Poultice, } \\
\text { decoction }\end{array}$ & $\begin{array}{l}\text { Itching skin, kidney } \\
\text { pain, colic, bloating }\end{array}$ & 4 & 0.16 & 25 & 0.07 & 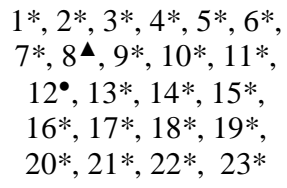 & \\
\hline
\end{tabular}




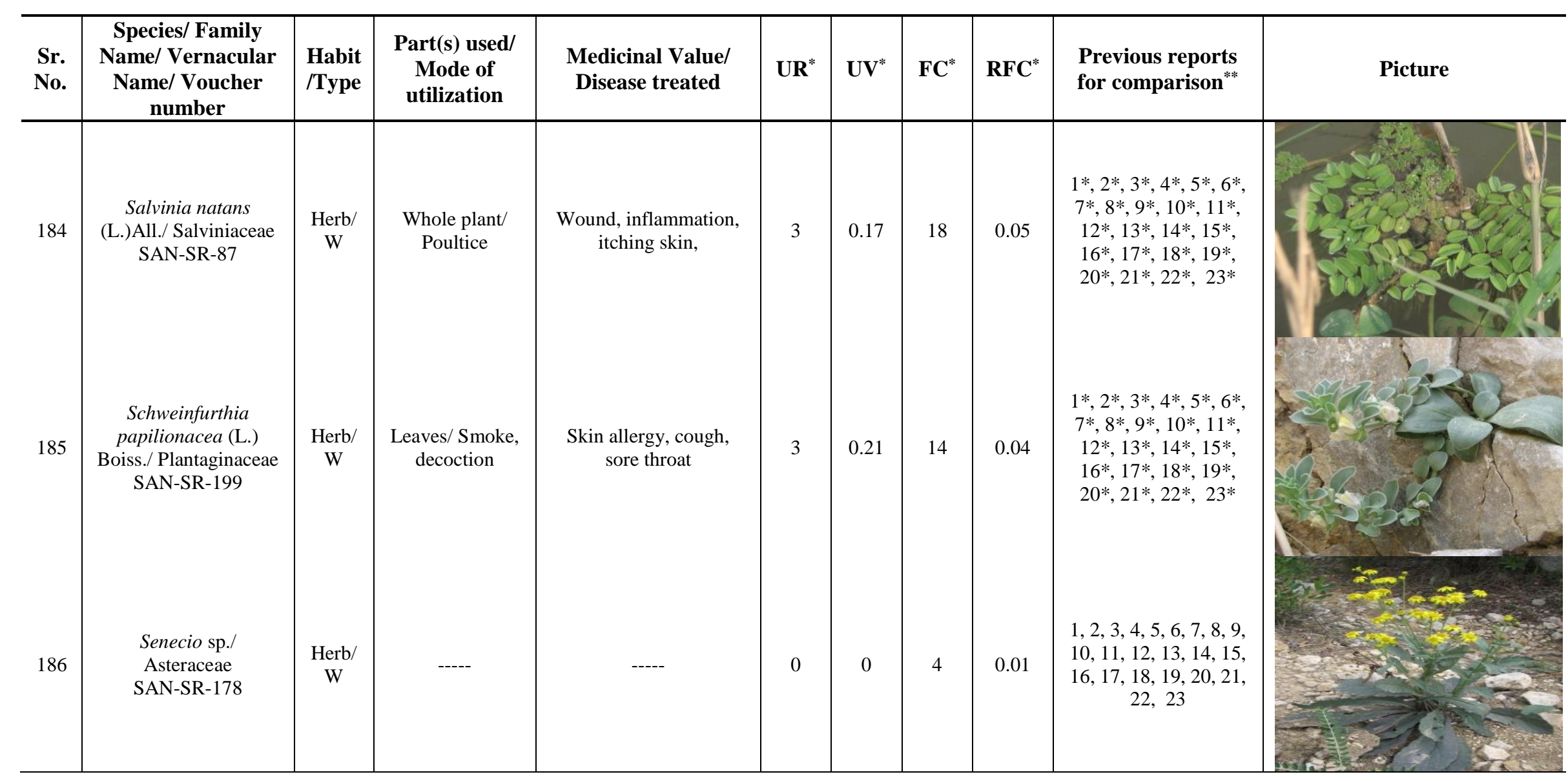




\begin{tabular}{|c|c|c|c|c|c|c|c|c|c|c|}
\hline $\begin{array}{l}\text { Sr. } \\
\text { No. }\end{array}$ & $\begin{array}{c}\text { Species/ Family } \\
\text { Name/ Vernacular } \\
\text { Name/ Voucher } \\
\text { number } \\
\end{array}$ & $\begin{array}{l}\text { Habit } \\
\text { /Type }\end{array}$ & $\begin{array}{l}\text { Part(s) used/ } \\
\text { Mode of } \\
\text { utilization }\end{array}$ & $\begin{array}{l}\text { Medicinal Value/ } \\
\text { Disease treated }\end{array}$ & $\mathbf{U R}^{*}$ & $\mathbf{U V}^{*}$ & $\mathbf{F C}^{*}$ & RFC $^{*}$ & $\begin{array}{l}\text { Previous reports } \\
\text { for comparison** }\end{array}$ & Picture \\
\hline 187 & $\begin{array}{c}\text { Sisymbrium irio L./ } \\
\text { Brassicaceae } \\
\text { Khoob kalan } \\
\text { SAN-SR-170 }\end{array}$ & $\begin{array}{c}\text { Herb/ } \\
\text { W }\end{array}$ & $\begin{array}{l}\text { Flowers/ } \\
\text { Decoction }\end{array}$ & $\begin{array}{l}\text { Chest infections, } \\
\text { expectorant }\end{array}$ & 2 & 0.08 & 23 & 0.06 & $\begin{array}{c}1^{*}, 2^{*}, 3^{*}, 4^{*}, 5^{*}, 6^{*}, \\
7^{*}, 8^{*}, 9^{*}, 10^{*}, 11^{*} \\
12^{*}, 13^{*}, 14^{*}, 15^{*} \\
16^{*}, 17^{*}, 18^{*}, 19^{\star} \\
20^{*}, 21^{*}, 22^{*}, 23^{*}\end{array}$ & \\
\hline 188 & $\begin{array}{c}\text { Solanum } \\
\text { elaeagnifolium Cav./ } \\
\text { Solanaceae } \\
\text { SAN-SR-192 }\end{array}$ & $\begin{array}{c}\text { Herb/ } \\
\text { W }\end{array}$ & $\begin{array}{l}\text { Fruits, flower/ } \\
\text { Infusion }\end{array}$ & $\begin{array}{l}\text { Analgesic, toothache, } \\
\text { menstrual pain }\end{array}$ & 3 & 0.14 & 22 & 0.06 & $\begin{array}{c}1^{*}, 2^{*}, 3^{*}, 4^{*}, 5^{*}, 6^{*} \\
7^{*}, 8^{*}, 9^{*}, 10^{*}, 11^{*} \\
12^{*}, 13^{*}, 14^{*}, 15^{*} \\
16^{*}, 17^{*}, 18^{*}, 19^{*} \\
20^{*}, 21^{*}, 22^{*}, 23^{*}\end{array}$ & \\
\hline 189 & $\begin{array}{l}\text { Solanum incanum L./ } \\
\text { Solanaceae } \\
\text { SAN-SR-105 }\end{array}$ & $\begin{array}{l}\text { Herb/ } \\
\text { W }\end{array}$ & $\begin{array}{l}\text { Fruits, flower/ } \\
\text { Infusion }\end{array}$ & $\begin{array}{l}\text { Respiratory problems, } \\
\text { gastrointestinal } \\
\text { problems }\end{array}$ & 2 & 0.14 & 14 & 0.04 & $\begin{array}{c}1^{*}, 2^{*}, 3^{*}, 4^{*}, 5^{*}, 6^{*}, \\
7^{*}, 8^{*}, 9^{*}, 10^{*}, 11^{*} \\
12^{*}, 13^{*}, 14^{*}, 15^{*} \\
16^{*}, 17^{*}, 18^{*}, 19^{*} \\
20^{*}, 21^{*}, 22^{*}, 23^{*}\end{array}$ & \\
\hline
\end{tabular}




\begin{tabular}{|c|c|c|c|c|c|c|c|c|c|c|}
\hline $\begin{array}{l}\text { Sr. } \\
\text { No. }\end{array}$ & $\begin{array}{c}\text { Species/ Family } \\
\text { Name/ Vernacular } \\
\text { Name/ Voucher } \\
\text { number } \\
\end{array}$ & $\begin{array}{l}\text { Habit } \\
\text { /Type }\end{array}$ & $\begin{array}{l}\text { Part(s) used/ } \\
\text { Mode of } \\
\text { utilization }\end{array}$ & $\begin{array}{l}\text { Medicinal Value/ } \\
\text { Disease treated }\end{array}$ & $\mathbf{U R}^{*}$ & $\mathbf{U} \mathbf{V}^{*}$ & $\mathbf{F C}^{*}$ & RFC $^{*}$ & $\begin{array}{l}\text { Previous reports } \\
\text { for comparison** }\end{array}$ & Picture \\
\hline 190 & $\begin{array}{c}\text { Solanum nigrum } \mathrm{L} . / \\
\text { Solanaceae } \\
\text { Mako } \\
\text { SAN-SR-30 }\end{array}$ & $\begin{array}{l}\text { Herb/ } \\
\text { W }\end{array}$ & $\begin{array}{l}\text { Fruits, seeds/ } \\
\text { Decoction }\end{array}$ & $\begin{array}{l}\text { Hepatitis, respiratory } \\
\text { problems, body pain }\end{array}$ & 3 & 0.19 & 16 & 0.04 & $\begin{array}{l}1^{\bullet}, 2^{\bullet}, 3^{\bullet}, 4^{*}, 5^{\bullet}, 6^{*} \\
7^{\bullet}, 8^{\mathbf{4}}, 9^{*}, 10^{*}, 11^{*} \\
12^{*}, 13^{*}, 14^{\mathbf{4}}, 15^{*} \\
16^{*}, 17^{*}, 18^{*}, 19^{*} \\
20^{\mathbf{4}}, 21^{*}, 22^{*}, 23^{*}\end{array}$ & \\
\hline 191 & $\begin{array}{c}\text { Solanum surattense } \\
\text { Burm. f./ Solanaceae } \\
\text { Mahorri } \\
\text { SAN-SR-131 }\end{array}$ & $\begin{array}{l}\text { Herb/ } \\
\text { W }\end{array}$ & Fruit/ Juice, raw & $\begin{array}{l}\text { Respiratory problems, } \\
\text { febrifuge, pain }\end{array}$ & 3 & 0.19 & 16 & 0.04 & $\begin{array}{c}1^{\mathbf{4}}, 2^{*}, 3^{*}, 4^{*}, 5^{*}, 6^{*} \\
7^{*}, 8^{\mathbf{4}}, 9^{*}, 10^{*}, 11^{*} \\
12^{*}, 13^{*}, 14^{*}, 15^{*} \\
16^{*}, 17^{*}, 18^{*}, 19^{*} \\
20^{*}, 21^{*}, 22^{*}, 23^{*}\end{array}$ & \\
\hline 192 & $\begin{array}{c}\text { Solanum } \\
\text { xanthocarpum Schrad. } \\
\text { \& H. Wendl./ } \\
\text { Solanaceae } \\
\text { Mahorri } \\
\text { SAN-SR-48 }\end{array}$ & $\begin{array}{l}\text { Herb/ } \\
\text { W }\end{array}$ & $\begin{array}{l}\text { Fruit, leaves/ } \\
\text { Decoction, } \\
\text { infusion }\end{array}$ & $\begin{array}{l}\text { Cough, cold, malaria, } \\
\text { headache, febrifuge }\end{array}$ & 5 & 0.28 & 18 & 0.05 & $\begin{array}{c}1^{*}, 2^{*}, 3^{*}, 4^{*}, 5^{*}, 6^{*}, \\
7^{*}, 8^{*}, 9^{*}, 10^{*}, 11^{*} \\
12^{*}, 13^{*}, 14^{*}, 15^{*} \\
16^{*}, 17^{*}, 18^{*}, 19^{*} \\
20^{*}, 21^{*}, 22^{*}, 23^{*}\end{array}$ & \\
\hline
\end{tabular}




\begin{tabular}{|c|c|c|c|c|c|c|c|c|c|c|}
\hline $\begin{array}{l}\text { Sr. } \\
\text { No. }\end{array}$ & $\begin{array}{c}\text { Species/ Family } \\
\text { Name/ Vernacular } \\
\text { Name/ Voucher } \\
\text { number } \\
\end{array}$ & $\begin{array}{l}\text { Habit } \\
\text { /Type }\end{array}$ & $\begin{array}{l}\text { Part(s) used/ } \\
\text { Mode of } \\
\text { utilization }\end{array}$ & $\begin{array}{l}\text { Medicinal Value/ } \\
\text { Disease treated }\end{array}$ & $\mathbf{U} \mathbf{R}^{*}$ & $\mathbf{U V}^{*}$ & $\mathbf{F C}^{*}$ & RFC $^{*}$ & $\begin{array}{l}\text { Previous reports } \\
\text { for comparison** }\end{array}$ & Picture \\
\hline 193 & $\begin{array}{c}\text { Sonchus asper }(\mathrm{L} .) \\
\text { Hill/ Asteraceae } \\
\text { Dhodak } \\
\text { SAN-SR-59 }\end{array}$ & $\begin{array}{l}\text { Herb/ } \\
\text { W }\end{array}$ & $\begin{array}{l}\text { Whole plant/ } \\
\text { Decoction }\end{array}$ & $\begin{array}{l}\text { Stomach ache, head } \\
\text { ache, migraine }\end{array}$ & 3 & 0.2 & 15 & 0.04 & $\begin{array}{c}1^{*}, 2^{*}, 3^{\mathbf{4}}, 4^{*}, 5^{\mathbf{\Lambda}}, 6^{*}, \\
7^{*}, 8^{\mathbf{4}}, 9^{*}, 10^{*}, 11^{*}, \\
12^{*}, 13^{*}, 14^{*}, 15^{*} \\
16^{*}, 17^{*}, 18^{*}, 19^{*} \\
20^{*}, 21^{*}, 22^{*}, 23^{*}\end{array}$ & $x^{2}$ \\
\hline 194 & $\begin{array}{c}\text { Stellaria media }(\mathrm{L} .) \\
\text { Vill./ Caryophyllaceae } \\
\text { Banbatorr } \\
\text { SAN-SR-50 }\end{array}$ & $\begin{array}{c}\text { Herb/ } \\
\text { W }\end{array}$ & $\begin{array}{l}\text { Aerial parts/ } \\
\text { Paste, infusion }\end{array}$ & $\begin{array}{l}\text { ENT problems, piles, } \\
\text { jaundice }\end{array}$ & 3 & 0.21 & 14 & 0.04 & $\begin{array}{c}1^{*}, 2^{\star}, 3^{*}, 4^{*}, 5^{*}, 6^{*}, \\
7^{*}, 8^{*}, 9^{*}, 10^{*}, 11^{*}, \\
12^{*}, 13^{*}, 14^{*}, 15^{*} \\
16^{*}, 17^{*}, 18^{*}, 19^{*} \\
20^{*}, 21^{*}, 22^{*}, 23^{*}\end{array}$ & \\
\hline 195 & $\begin{array}{c}\text { Tamarix aphylla }(\mathrm{L} .) \\
\text { H. Karst./ } \\
\text { Tamaricaceae } \\
\text { Khaggal } \\
\text { SAN-SR-114 }\end{array}$ & $\begin{array}{l}\text { Tree/ } \\
\mathrm{W}\end{array}$ & Leaves/ Smoke & $\begin{array}{l}\text { Measles, respiratory } \\
\text { disorders, febrifuge, } \\
\text { body pain }\end{array}$ & 4 & 0.10 & 39 & 0.11 & $\begin{array}{c}1^{*}, 2^{*}, 3^{*}, 4^{*}, 5^{*}, 6^{*}, \\
7^{*}, 8^{*}, 9^{*}, 10^{*}, 11^{*} \\
12^{*}, 13^{*}, 14^{*}, 15^{*} \\
16^{*}, 17^{*}, 18^{*}, 19^{*} \\
20^{*}, 21^{*}, 22^{*}, 23^{*}\end{array}$ & \\
\hline 196 & $\begin{array}{c}\text { Tamarix dioica Roxb. } \\
\text { Ex Roth./ } \\
\text { Tamaricaceae } \\
\text { Khaggal } \\
\text { SAN-SR-94 }\end{array}$ & $\begin{array}{l}\text { Tree/ } \\
\mathrm{W}\end{array}$ & Leaves/ Smoke & Measles & 4 & 0.09 & 42 & 0.12 & $\begin{array}{c}1^{*}, 2^{*}, 3^{*}, 4^{*}, 5^{*}, 6^{*}, \\
7^{*}, 8^{*}, 9^{*}, 10^{*}, 11^{*} \\
12^{*}, 13^{*}, 14^{*}, 15^{*} \\
16^{*}, 17^{*}, 18^{*}, 19^{*} \\
20^{*}, 21^{*}, 22^{*}, 23^{*}\end{array}$ & \\
\hline
\end{tabular}

APPLIED ECOLOGY AND ENVIRONMENTAL RESEARCH 17(2):4725-4805.

http://www.aloki.hu • ISSN 15891623 (Print) • ISSN 17850037 (Online)

DOI: http://dx.doi.org/10.15666/aeer/1702_47254805

(c) 2019, ALÖKI Kft., Budapest, Hungary 


\begin{tabular}{|c|c|c|c|c|c|c|c|c|c|c|}
\hline $\begin{array}{l}\text { Sr. } \\
\text { No. }\end{array}$ & $\begin{array}{c}\text { Species/ Family } \\
\text { Name/ Vernacular } \\
\text { Name/ Voucher } \\
\text { number } \\
\end{array}$ & $\begin{array}{l}\text { Habit } \\
\text { /Type }\end{array}$ & $\begin{array}{l}\text { Part(s) used/ } \\
\text { Mode of } \\
\text { utilization }\end{array}$ & $\begin{array}{l}\text { Medicinal Value/ } \\
\text { Disease treated }\end{array}$ & $\mathbf{U R}^{*}$ & $\mathbf{U} \mathbf{V}^{*}$ & $\mathbf{F C}^{*}$ & RFC $^{*}$ & $\begin{array}{l}\text { Previous reports } \\
\text { for comparison** }\end{array}$ & Picture \\
\hline 197 & $\begin{array}{l}\text { Taraxacum officinale } \\
\text { (L.) Weber F. H. } \\
\text { Wigg./ Asteraceae } \\
\text { Duddal } \\
\text { SAN-SR-116 }\end{array}$ & $\begin{array}{c}\text { Herb/ } \\
\text { W }\end{array}$ & Flower/ Infusion & $\begin{array}{c}\text { Stomach problems, } \\
\text { hepatitis, }\end{array}$ & 2 & 0.09 & 22 & 0.06 & 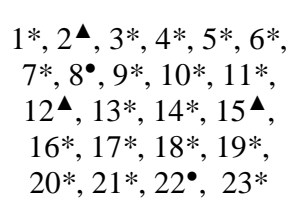 & \\
\hline 198 & $\begin{array}{c}\text { Taverniera glabra } \\
\text { Boiss./ Fabaceae } \\
\text { SAN-SR-117 }\end{array}$ & $\begin{array}{c}\text { Shrub/ } \\
\text { W }\end{array}$ & $\begin{array}{l}\text { Leaves, bark/ } \\
\text { Decoction }\end{array}$ & $\begin{array}{l}\text { Body pain, obesity, poor } \\
\text { appetite }\end{array}$ & 3 & 0.3 & 10 & 0.03 & $\begin{array}{c}1^{*}, 2^{*}, 3^{*}, 4^{*}, 5^{*}, 6^{*}, \\
7^{*}, 8^{*}, 9^{*}, 10^{*}, 11^{*} \\
12^{*}, 13^{*}, 14^{*}, 15^{*} \\
16^{*}, 17^{*}, 18^{*}, 19^{*} \\
20^{*}, 21^{*}, 22^{*}, 23^{*}\end{array}$ & \\
\hline 199 & $\begin{array}{c}\text { Taverniera spartea } \\
\text { (Burm.f.) DC./ } \\
\text { Fabaceae } \\
\text { SAN-SR-137 }\end{array}$ & $\begin{array}{l}\text { Shrub/ } \\
\text { W }\end{array}$ & Stem/ Decoction & $\begin{array}{c}\text { Cancer, blood } \\
\text { purification, pimples }\end{array}$ & 3 & 0.21 & 14 & 0.04 & $\begin{array}{c}1^{*}, 2^{*}, 3^{*}, 4^{*}, 5^{*}, 6^{*}, \\
7^{*}, 8^{*}, 9^{*}, 10^{*}, 11^{*}, \\
12^{*}, 13^{*}, 14^{*}, 15^{*} \\
16^{*}, 17^{*}, 18^{*}, 19^{*} \\
20^{*}, 21^{*}, 22^{*}, 23^{*}\end{array}$ & \\
\hline
\end{tabular}




\begin{tabular}{|c|c|c|c|c|c|c|c|c|c|c|}
\hline $\begin{array}{l}\text { Sr. } \\
\text { No. }\end{array}$ & $\begin{array}{c}\text { Species/ Family } \\
\text { Name/ Vernacular } \\
\text { Name/ Voucher } \\
\text { number } \\
\end{array}$ & $\begin{array}{l}\text { Habit } \\
\text { /Type }\end{array}$ & $\begin{array}{l}\text { Part(s) used/ } \\
\text { Mode of } \\
\text { utilization }\end{array}$ & $\begin{array}{l}\text { Medicinal Value/ } \\
\text { Disease treated }\end{array}$ & $\mathbf{U} \mathbf{R}^{*}$ & $\mathbf{U V}^{*}$ & $\mathbf{F C}^{*}$ & RFC $^{*}$ & $\begin{array}{l}\text { Previous reports } \\
\text { for comparison** }\end{array}$ & Picture \\
\hline 200 & $\begin{array}{c}\text { Tecomella undulata } \\
\text { (sm.) Seem./ } \\
\text { Bignoniaceae } \\
\text { Roheerra } \\
\text { SAN-SR-141 }\end{array}$ & $\begin{array}{l}\text { Tree/ } \\
\text { W }\end{array}$ & Bark/ Decoction & Urinary problems, & 1 & 0.08 & 12 & 0.03 & $\begin{array}{c}1^{*}, 2^{*}, 3^{*}, 4^{*}, 5^{*}, 6^{*}, \\
7^{*}, 8^{*}, 9^{*}, 10^{*}, 11^{*} \\
12^{*}, 13^{*}, 14^{*}, 15^{*} \\
16^{*}, 17^{*}, 18^{*}, 19^{*} \\
20^{*}, 21^{*}, 22^{*}, 23^{*}\end{array}$ & \\
\hline 201 & $\begin{array}{c}\text { Tephrosia apollinea } \\
\text { (Delile) DC./ Fabaceae } \\
\text { SAN-SR-144 }\end{array}$ & $\begin{array}{l}\text { Herb/ } \\
\text { W }\end{array}$ & $\begin{array}{l}\text { Pods, flower/ } \\
\text { Decoction }\end{array}$ & $\begin{array}{c}\text { Inflammation, } \\
\text { toothache, respiratory } \\
\text { problems }\end{array}$ & 3 & 0.21 & 14 & 0.04 & $\begin{array}{c}1^{*}, 2^{*}, 3^{*}, 4^{*}, 5^{*}, 6^{*}, \\
7^{*}, 8^{*}, 9^{*}, 10^{*}, 11^{*} \\
12^{*}, 13^{*}, 14^{*}, 15^{*} \\
16^{*}, 17^{*}, 18^{*}, 19^{*} \\
20^{*}, 21^{*}, 22^{*}, 23^{*}\end{array}$ & \\
\hline 202 & $\begin{array}{c}\text { Trianthema } \\
\text { portulacastrum } \text { L./ } \\
\text { Aizoaceae } \\
\text { It sit } \\
\text { SAN-SR-151 }\end{array}$ & $\begin{array}{l}\text { Herb/ } \\
\text { W }\end{array}$ & Leaves/Juice & $\begin{array}{c}\text { Antidote, } \\
\text { gynaecological } \\
\text { disorders, stomach } \\
\text { problems, eye infections }\end{array}$ & 4 & 0.14 & 28 & 0.08 & $\begin{array}{c}1^{\star}, 2^{*}, 3^{*}, 4^{*}, 5^{*}, 6^{*} \\
7^{*}, 8^{*}, 9^{*}, 10^{*}, 11^{*} \\
12^{*}, 13^{*}, 14^{*}, 15^{*} \\
16^{*}, 17^{*}, 18^{*}, 19^{*} \\
20^{*}, 21^{*}, 22^{*}, 23^{*}\end{array}$ & \\
\hline
\end{tabular}

APPLIED ECOLOGY AND ENVIRONMENTAL RESEARCH 17(2):4725-4805.

http://www.aloki.hu • ISSN 15891623 (Print) • ISSN 17850037 (Online)

DOI: http://dx.doi.org/10.15666/aeer/1702_47254805

(c) 2019, ALÖKI Kft., Budapest, Hungary 


\begin{tabular}{|c|c|c|c|c|c|c|c|c|c|c|}
\hline $\begin{array}{l}\text { Sr. } \\
\text { No. }\end{array}$ & $\begin{array}{c}\text { Species/ Family } \\
\text { Name/ Vernacular } \\
\text { Name/ Voucher } \\
\text { number } \\
\end{array}$ & $\begin{array}{l}\text { Habit } \\
\text { /Type }\end{array}$ & $\begin{array}{l}\text { Part(s) used/ } \\
\text { Mode of } \\
\text { utilization }\end{array}$ & $\begin{array}{l}\text { Medicinal Value/ } \\
\text { Disease treated }\end{array}$ & $\mathbf{U} \mathbf{R}^{*}$ & $\mathbf{U V}^{*}$ & $\mathbf{F C}^{*}$ & RFC $^{*}$ & $\begin{array}{l}\text { Previous reports } \\
\text { for comparison }{ }^{* *}\end{array}$ & Picture \\
\hline 203 & $\begin{array}{l}\text { Tribulus terrestris L./ } \\
\text { Zygophyllaceae } \\
\text { Bhakrra } \\
\text { SAN-SR-155 }\end{array}$ & $\begin{array}{l}\text { Herb/ } \\
\text { W }\end{array}$ & $\begin{array}{l}\text { Aerial parts/ } \\
\text { Decoction }\end{array}$ & $\begin{array}{c}\text { Aphrodisiac, respiratory } \\
\text { problems, febrifuge, } \\
\text { headache, laxative }\end{array}$ & 5 & 0.14 & 36 & 0.10 & 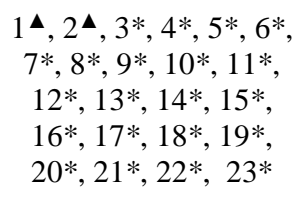 & \\
\hline 204 & $\begin{array}{l}\text { Trichodesma indicum } \\
\text { (L.) Lehm./ } \\
\text { Boraginaceae }\end{array}$ & $\begin{array}{c}\text { Herb/ } \\
\text { W }\end{array}$ & $\begin{array}{l}\text { Leaves/ } \\
\text { Decoction, } \\
\text { infusion }\end{array}$ & $\begin{array}{c}\text { Antidote, laxative, body } \\
\text { pain, fever }\end{array}$ & 4 & 0.2 & 20 & 0.05 & $\begin{array}{c}1^{*}, 2^{*}, 3^{*}, 4^{*}, 5^{*}, 6^{*} \\
7^{*}, 8^{\mathbf{4}}, 9^{*}, 10^{*}, 11^{*} \\
12^{*}, 13^{*}, 14^{*}, 15^{*} \\
16^{*}, 17^{*}, 18^{*}, 19^{*} \\
20^{*}, 21^{*}, 22^{*}, 23^{*}\end{array}$ & \\
\hline 205 & $\begin{array}{c}\text { Typha angustata Bory } \\
\text { \& Chaub./ Typhaceae } \\
\text { Koondar } \\
\text { SAN-SR-158 }\end{array}$ & $\begin{array}{c}\text { Herb/ } \\
\text { W }\end{array}$ & $\begin{array}{l}\text { Whole plant/ } \\
\text { Decoction }\end{array}$ & $\begin{array}{l}\text { Respiratory disorders, } \\
\text { stomach problems, } \\
\text { gynaecological } \\
\text { problems }\end{array}$ & 3 & 0.07 & 39 & 0.11 & $\begin{array}{c}1^{*}, 2^{*}, 3^{*}, 4^{*}, 5^{*}, 6^{*}, \\
7^{*}, 8^{*}, 9^{*}, 10^{*}, 11^{*} \\
12^{*}, 13^{*}, 14^{*}, 15^{*} \\
16^{*}, 17^{*}, 18^{*}, 19^{*} \\
20^{*}, 21^{*}, 22^{*}, 23^{*}\end{array}$ & \\
\hline 206 & $\begin{array}{l}\text { Typha latifolia } \text { L./ } \\
\text { Typhaceae } \\
\text { Koondar } \\
\text { SAN-SR-165 }\end{array}$ & $\begin{array}{c}\text { Herb/ } \\
\text { W }\end{array}$ & $\begin{array}{l}\text { Whole plant/ } \\
\text { Decoction }\end{array}$ & $\begin{array}{l}\text { Respiratory disorders, } \\
\text { stomach problems, } \\
\text { gynaecological } \\
\text { problems }\end{array}$ & 3 & 0.07 & 38 & 0.11 & $\begin{array}{c}1^{*}, 2^{*}, 3^{*}, 4^{*}, 5^{*}, 6^{*}, \\
7^{*}, 8^{*}, 9^{*}, 10^{*}, 11^{*}, \\
12^{*}, 13^{*}, 14^{*}, 15^{*} \\
16^{*}, 17^{*}, 18^{*}, 19^{*} \\
20^{*}, 21^{*}, 22^{*}, 23^{*}\end{array}$ & \\
\hline
\end{tabular}

APPLIED ECOLOGY AND ENVIRONMENTAL RESEARCH 17(2):4725-4805.

http://www.aloki.hu • ISSN 15891623 (Print) • ISSN 17850037 (Online)

DOI: http://dx.doi.org/10.15666/aeer/1702_47254805

(c) 2019, ALÖKI Kft., Budapest, Hungary 


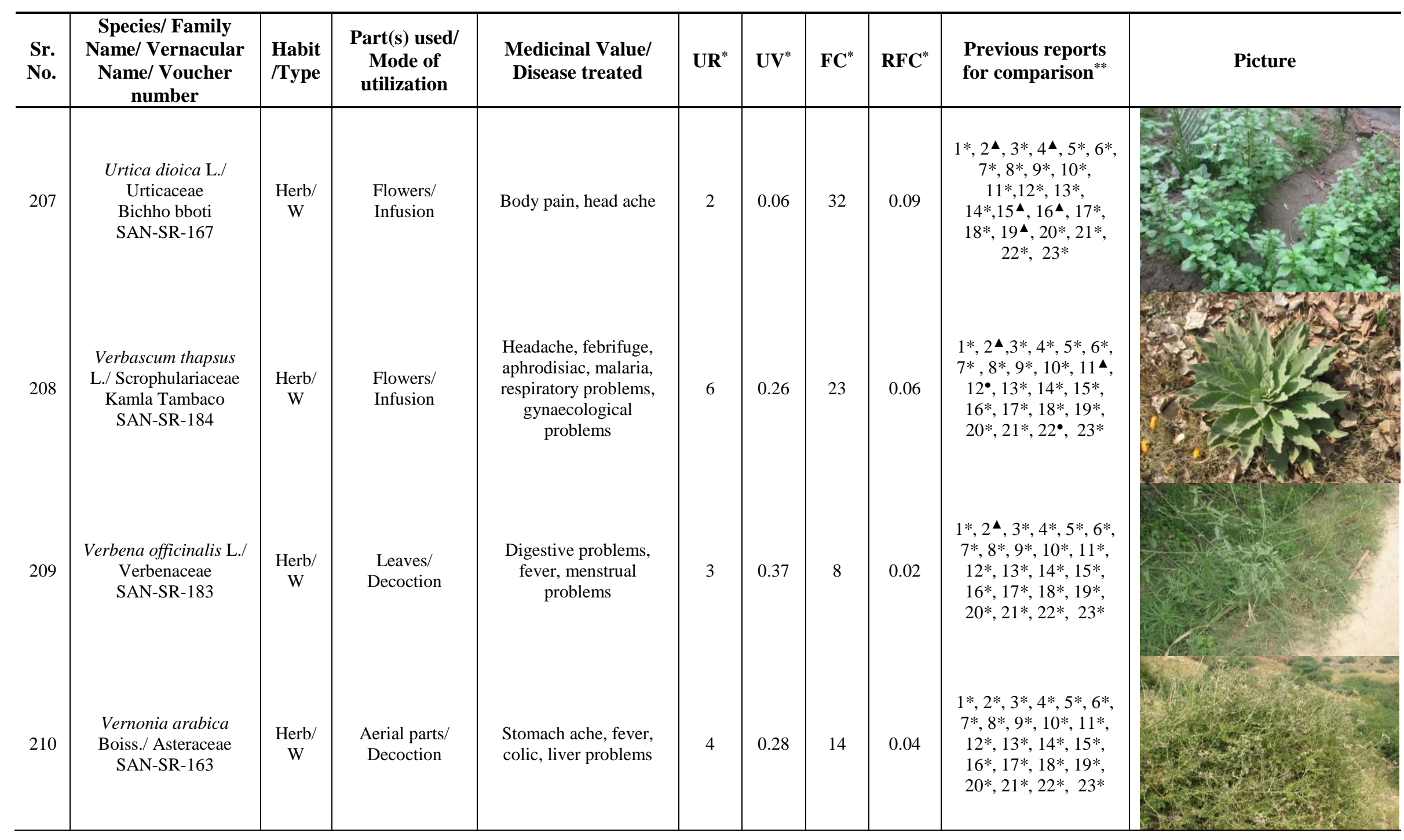

APPLIED ECOLOGY AND ENVIRONMENTAL RESEARCH 17(2):4725-4805.

http://www.aloki.hu • ISSN 15891623 (Print) • ISSN 17850037 (Online)

DOI: http://dx.doi.org/10.15666/aeer/1702_47254805

(c) 2019, ALÖKI Kft., Budapest, Hungary 


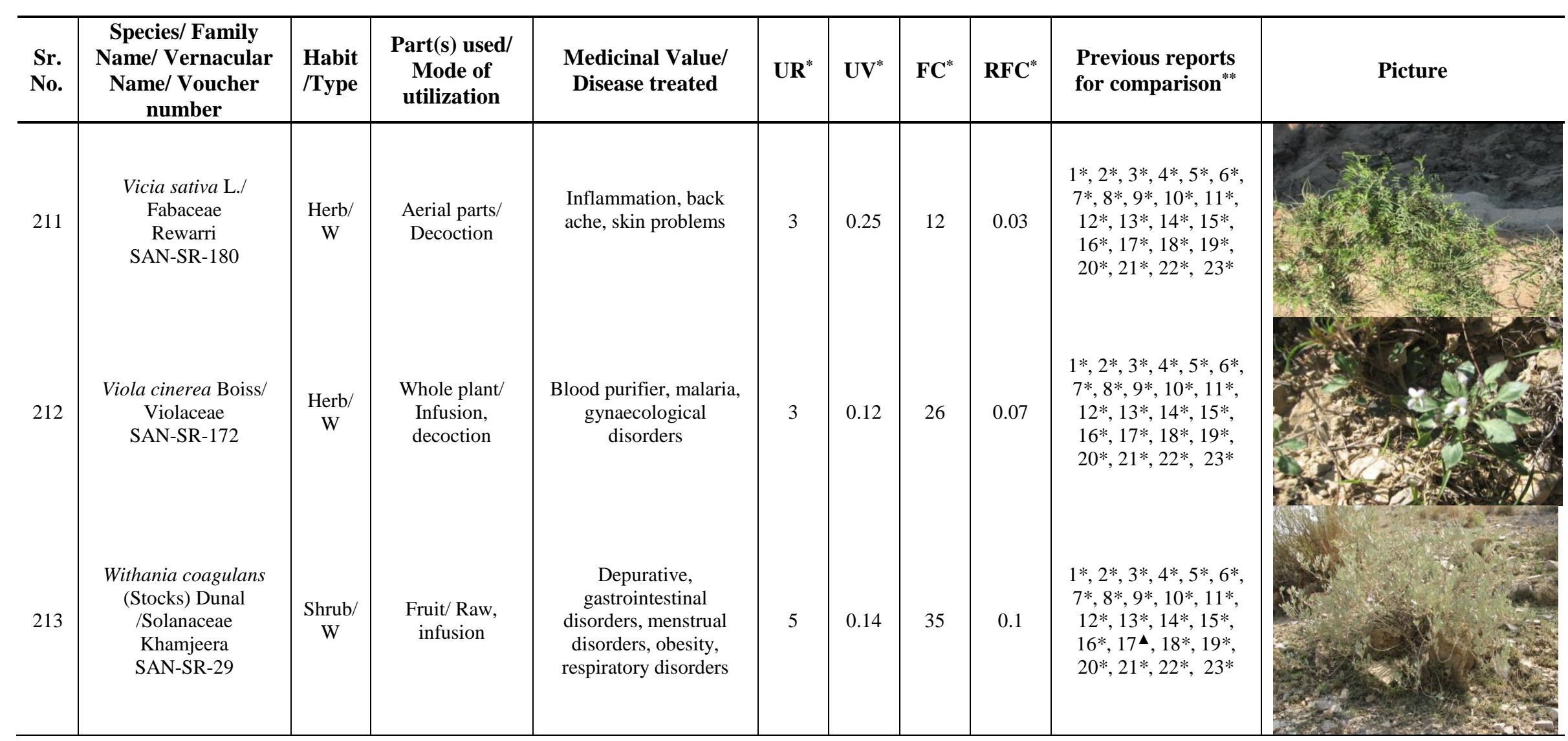




\begin{tabular}{|c|c|c|c|c|c|c|c|c|c|c|}
\hline $\begin{array}{l}\text { Sr. } \\
\text { No. }\end{array}$ & $\begin{array}{c}\text { Species/ Family } \\
\text { Name/ Vernacular } \\
\text { Name/ Voucher } \\
\text { number } \\
\end{array}$ & $\begin{array}{l}\text { Habit } \\
\text { /Type }\end{array}$ & $\begin{array}{l}\text { Part(s) used/ } \\
\text { Mode of } \\
\text { utilization }\end{array}$ & $\begin{array}{l}\text { Medicinal Value/ } \\
\text { Disease treated }\end{array}$ & $\mathbf{U R}^{*}$ & $\mathbf{U V}^{*}$ & $\mathbf{F C}^{*}$ & RFC $^{*}$ & $\begin{array}{l}\text { Previous reports } \\
\text { for comparison** }\end{array}$ & Picture \\
\hline 214 & $\begin{array}{c}\text { Withania somnifera } \\
\text { (L). Dunal /Solanaceae } \\
\text { Kutai lal } \\
\text { SAN-SR-193 }\end{array}$ & $\begin{array}{l}\text { Herb/ } \\
\text { W }\end{array}$ & Fruit/ Infusion & $\begin{array}{l}\text { Abdominal pain, } \\
\text { gastrointestinal } \\
\text { problems, vomiting, } \\
\text { skin problems, } \\
\text { depurative, cough, cold, } \\
\text { throat infections, } \\
\text { insomnia }\end{array}$ & 9 & 0.25 & 35 & 0.1 & $\begin{array}{c}1^{*}, 2^{\mathbf{4}}, 3,4,5^{\mathbf{4}}, 6^{*} \\
7^{*}, 8^{\mathbf{4}}, 9^{*}, 10^{*}, 11^{*}, \\
12^{*}, 13^{\mathbf{4}}, 14^{*}, 15^{*}, \\
16^{*}, 17^{\bullet}, 18^{*}, 19^{*}, \\
20^{*}, 21^{*}, 22^{\mathbf{4}}, 23^{\bullet}\end{array}$ & \\
\hline 215 & $\begin{array}{c}\text { Xanthium strumarium } \\
\text { L./ Asteraceae } \\
\text { Chhota Dhatura } \\
\text { SAN-SR-187 }\end{array}$ & $\begin{array}{c}\text { Herb/ } \\
\text { W }\end{array}$ & $\begin{array}{l}\text { Leaves, aerial } \\
\text { parts/ Infusion, } \\
\text { powder }\end{array}$ & $\begin{array}{c}\text { Oral infection, } \\
\text { dysmenorrhea, } \\
\text { hypertension, malaria }\end{array}$ & 4 & 0.31 & 13 & 0.04 & 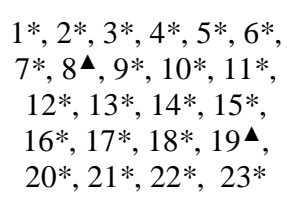 & \\
\hline
\end{tabular}




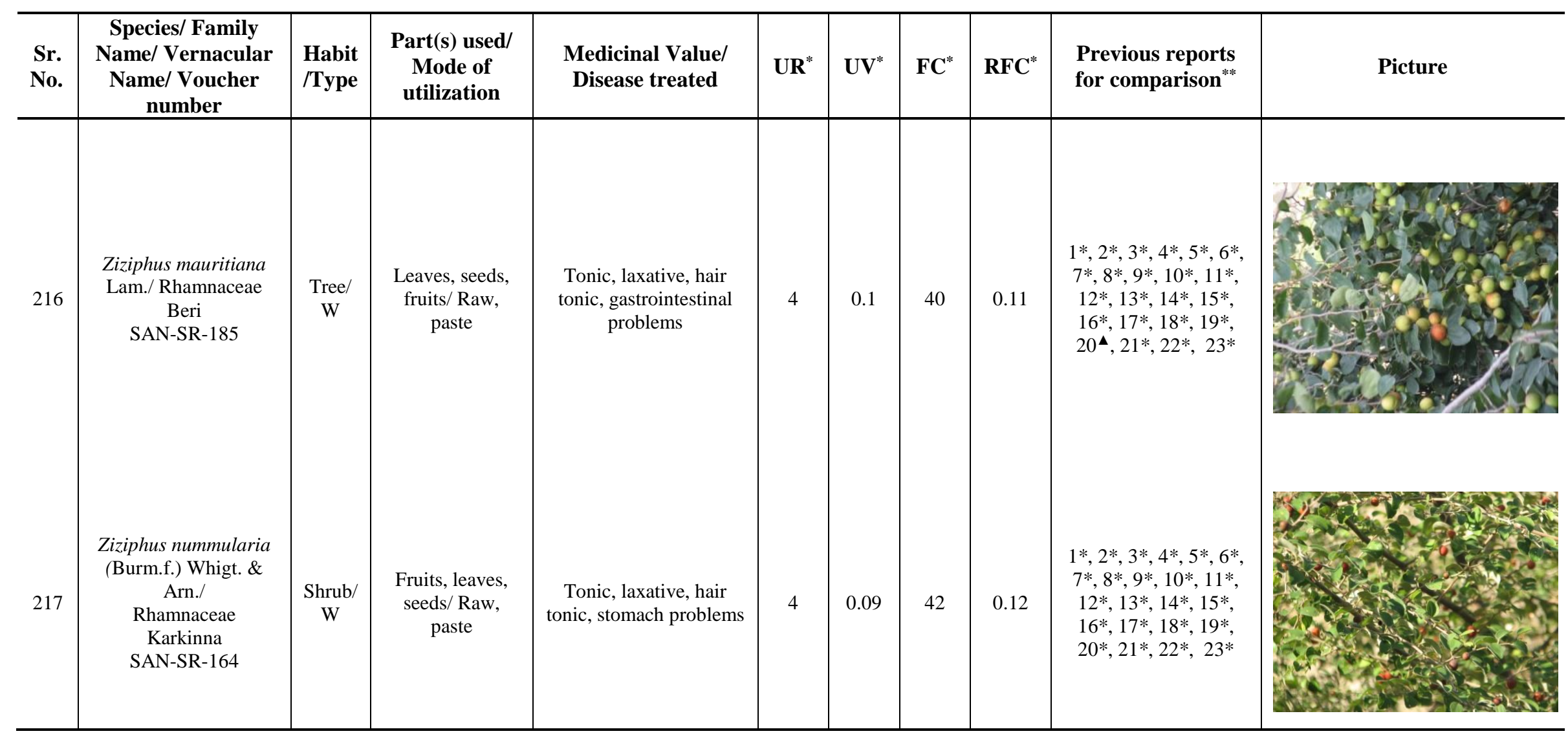

$1=$ Yabesh et al. (2014); 2= Haq. (2012); 3= Khan et al. (2010); 4= Phondani et al. (2010); 5= Zabihullah et al. (2006); 6= Hazrat et al. (2011); 7= Khan and Khatoon (2008); 8= Amjad. (2015); 9= Jiofack et al. (2009); 10= Bano et al. (2014); 11= Ahmad et al. (2014); 12= Khan et al. (2013); 13= Belayneh et al. (2012); 14= Teklehaymanot and Giday. (2010); 15= Pieroni and Giusti. (2009); 16= Pradhan and Badola. (2008); 17= Shah and Rahim. (2017); 18= Tetali et al. (2009); 19= Ali and Qaiser. (2009); 20= Rasingam. (2012); 21= Sanz-Biset et al. (2009); 22= Sharma et al. (2004); 23= Teklehaymanot and Giday. (2010)

$\mathrm{UR}^{*}$; Use Report, VU*; Use Value, FC*; frequency of Citation, $\mathrm{RFC}^{*}$; Relative Frequency Citation

W; Wild, C; Cultivated

${ }^{* *}(\bullet)$ - Similar use, $(\boldsymbol{\Lambda})$ - Dissimilar use, $(*)$ - Use not reported 


\section{Collection and taxonomic identification of plants}

Plants were collected with the help of traditional healer and local respondents just after the formal and informal discussion on ethnobotanically important plants of the area. Local informants guided researchers to the natural habitat of the discussed species and in some cases also helped to collect voucher specimens. After recording information about plants, voucher specimen were pressed in the field and dried properly. Plants were identified following the Flora of Pakistan (Nasir and Ali, 19702001). The list of plant species used ethnobotanically in the area was checked and updated after verification from the online website (http://www.theplantlist.org) of the Royle Botanic Garden, Kew, assessed on April 3, 2014. Voucher specimens were preserved and deposited in the Herbarium of University of Sargodha for their taxonomic identification by comparison with specimen in the herbarium and for future reference.

\section{Quantitative analyses of ethnobotanical data}

Ethnobotanically important plant species were arranged in alphabetic order by botanical name, family, habit, parts used, modes of utilization and disease treated. Different quantitative indices including use value (UV), frequency of citation (FC), relative frequency citation (RFC), family importance value (FIV), informant consensus factor (ICF) and the Jaccard index (JI) were applied to analyze the documented data.

\section{Use Value (UV)}

The UV is a quantitative index used to determine the relative importance of an indigenous plant species. According to Phillips et al. (1994), the use value was calculated by using the formula :

$$
U V=\sum U / n
$$

Where, "U" refers to the number of uses mentioned by the participant for a given species and " $n$ " refers to the total number of informants interviewed that used the given species. High UV score indicates that there are many use reports for that plant and vice versa.

\section{Frequency of Citation (FC) and Relative Frequency Citation (RFC)}

The Frequency of Citation (FC) is number of informants mentioning the use of plant species.

Moreover, Relative Frequency Citation (RFC) index was evaluated by using the formula :

$$
R F C=F C / N(0<R F C<1)
$$

$\mathrm{N}$ is the total number of informants participating in the study. The RFC index ranges from " 0 " when nobody referred to a plant as useful to " 1 " when all informants referred to as useful. (Vitalini et al., 2013).

\section{Informant Consensus Factor (ICF)}

Informant Consensus Factor (ICF) was calculated using the formula (Heinrich et al., 1998): 


$$
I C F=(N u r-N t) / N u r-1
$$

Where, "Nur" refers to the total number of use reports mentioned for a particular disease category and "Nt" refers to the total number of plant taxa used for particular disease category. This formula was opted to find out the homogeneity in the ethnomedicinal information documented from the traditional informants.

To calculate ICF, reported medicinal plant species were grouped into 16 categories of various diseases reported in the study area by the informants. ICF is useful to focus and to determine the consistency of the data concerning certain sorts of disease categories (Canales et al., 2005; Heinrich et al., 1998). The result of this consensus ranges from 0 to 1 . A high value (close to 1 ) predicts that plants are used by the high percentage of the informants for a number of illness related to that comprehensive category, whereas, the low value (close to 0) shows that the plants are selected arbitrarily for a few or a single complaint or that informants did not share or exchange facts and data about the usage of plants (Abu-Irmaileh and Afifi, 2003; Akerele, 1988; Kloutsos et al., 2001). Medicinal plants for which very few diseases are referred are supposed to be pharmacologically less active and thereby have low lower ICF values (Gazzaneo et al., 2005; Sharma et al., 2012; Teklehaymanot, 2009), Table 2.

\section{Jaccard Index (JI)}

Jaccard index is used to compare the reported data with that of other ethnobotanical studies conducted in other areas of Pakistan and abroad (González-Tejero et al., 2008). $\mathrm{JI}$ is calculated by using the formula :

$$
J I=c \times 100 / a+b-c
$$

Where, "a" is the recorded number of species of the study area "A"(Namal Valley) and " $b$ " is the documented number of species of the area " $B$ ". Where " $c$ " is the number of species common to both "A" and "B". As far as indigenous communities are concerned, " $a$ " is the number of species reported by indigenous community " $A$ ", " $b$ " is the number of species cited by the indigenous community " $\mathrm{B}$ " and the " $\mathrm{c}$ " is the number of species reported by both "A" and "B" communities, Table 2.

\section{Results}

A total of 350 local informants were interviewed. The age of informants ranged from 25 years to above 65 . Education wise, $24.3 \%$ informants were illiterate, $20 \%$ completed their five years education, $18.6 \%$ completed their eight years education and only $13.1 \%$ informants had higher education (University level). A total of 217 plant species belonging to 166 genera and 70 families were collected from the Valley. The ethnobotanical uses of plants (vernacular name, habit/type, part used/ mode of utilization, disease treated and other information like UR, UV, FC, RFC, previous report) are included in the Table 1. The highest number of reported species belong to family Fabaceae (19 species) followed by Asteraceae (18 species), Poaceae (13 species), Euphorbiaceae and Solanaceae (9 species each) and Lamiaceae and Moraceae (8 species each) etc. Most of the documented species were herbs $(71 \%)$, followed by shrubs (15\%) and trees (14\%) (Fig. 2). 
Table 2. Comparison of present study with previous reports at national and global level (JI*; Jaccard index)

\begin{tabular}{|c|c|c|c|c|c|c|c|c|c|c|c|c|}
\hline $\begin{array}{l}\text { Sr. } \\
\text { No. }\end{array}$ & Reference & Valley name/ Region & $\begin{array}{c}\begin{array}{c}\text { No. of } \\
\text { documented } \\
\text { plant } \\
\text { species }\end{array} \\
\end{array}$ & $\begin{array}{l}\text { Plants } \\
\text { with } \\
\text { similar } \\
\text { use }\end{array}$ & $\begin{array}{c}\text { Plants } \\
\text { with dis- } \\
\text { similar } \\
\text { use }\end{array}$ & $\begin{array}{c}\text { No. of } \\
\text { species } \\
\text { common } \\
\text { in both } \\
\text { areas }\end{array}$ & $\begin{array}{c}\text { \%age of } \\
\text { species } \\
\text { common } \\
\text { in both } \\
\text { areas }\end{array}$ & $\begin{array}{c}\text { Species } \\
\text { enlisted } \\
\text { only in } \\
\text { aligned } \\
\text { area }\end{array}$ & $\begin{array}{c}\text { Species } \\
\text { enlisted } \\
\text { only in } \\
\text { study } \\
\text { area }\end{array}$ & $\begin{array}{c}\text { \% age of } \\
\text { plants } \\
\text { with } \\
\text { similar } \\
\text { use }\end{array}$ & $\begin{array}{c}\% \text { age } \\
\text { of dis- } \\
\text { similar } \\
\text { uses }\end{array}$ & $\mathbf{J}^{{ }^{*} \mathbf{I}^{*}}$ \\
\hline 1 & Yabesh et al., 2014 & Silent Valley/ Kerala, India & 102 & 5 & 12 & 17 & 16.7 & 85 & 184 & 4.9 & 11.8 & 6.7 \\
\hline 2 & Haq, 2012 & $\begin{array}{c}\text { Allai Valley/ Western Himalaya } \\
\text { Pakistan }\end{array}$ & 172 & 15 & 17 & 32 & 18.6 & 140 & 169 & 8.7 & 9.9 & 11.5 \\
\hline 3 & Khan et al., 2010 & $\begin{array}{c}\text { Poonch Valley/ Azad Kashmir } \\
\text { (Pakistan) }\end{array}$ & 169 & 6 & 7 & 13 & 7.7 & 156 & 188 & 3.5 & 4.1 & 3.9 \\
\hline 4 & Phondani et al., 2010 & $\begin{array}{l}\text { Niti Valley/ Central Himalaya, } \\
\text { India }\end{array}$ & 86 & & 8 & 8 & 9.3 & 78 & 193 & 0 & 9.3 & 3.04 \\
\hline 5 & Zabihullah et al., 2006 & $\begin{array}{l}\text { Kot Manzaray Baba Valley/ } \\
\text { Malakand Agency, Pakistan }\end{array}$ & 82 & 9 & 15 & 24 & 29.3 & 58 & 177 & 10.1 & 18.3 & 11.4 \\
\hline 6 & Hazrat et al., 2011 & $\begin{array}{l}\text { Dir Kohistan Valley/ Khyber } \\
\text { Pukhtunkhwa, Pakistan. }\end{array}$ & 40 & 1 & 2 & 3 & 7.5 & 37 & 198 & 2.5 & 5 & 1.3 \\
\hline 7 & Khan and Khatoon, 2008 & $\begin{array}{c}\text { Haramosh and Bugrote Valleys/ } \\
\text { Gilgit, Pakistan }\end{array}$ & 98 & 6 & 5 & 11 & 11.2 & 87 & 190 & 6.1 & 5.1 & 4.1 \\
\hline 8 & Amjad, 2015 & Bana Valley/ Kotli, Pakistan & 86 & 12 & 28 & 40 & 46.5 & 46 & 161 & 13.9 & 32.5 & 23.9 \\
\hline 9 & Jiofack et al., 2009 & Upper Nyong Valley/ Cameroon & 140 & 0 & 3 & 3 & 2.1 & 137 & 198 & 0 & 2.14 & 0.9 \\
\hline 10 & Bano et al., 2014 & $\begin{array}{l}\text { Skardu Valley/ karakoram- } \\
\text { Himalayan range, Pakistan }\end{array}$ & 50 & 1 & 2 & 3 & 6 & 47 & 198 & 2 & 4 & 1.2 \\
\hline 11 & Ahmad et al., 2014 & Chail Valley/ Swat- Pakistan & 50 & 6 & 4 & 10 & 20 & 40 & 191 & 12 & 8 & 4.5 \\
\hline 12 & Khan et al., 2013 & $\begin{array}{c}\text { Naran Valley/ Western Himalaya, } \\
\text { Pakistan }\end{array}$ & 101 & 4 & 6 & 10 & 9.9 & 91 & 191 & 3.9 & 5.9 & 3.7 \\
\hline 13 & Belayneh et al., 2012 & Erer Valley/ Eastern Ethiopia & 51 & 2 & 1 & 3 & 5.9 & 48 & 198 & 3.9 & 1.9 & 1.23 \\
\hline 14 & $\begin{array}{l}\text { Teklehaymanot and } \\
\text { Giday, } 2010\end{array}$ & Lower Omo River Valley/ Ethiopia & 38 & 0 & 3 & 3 & 7.9 & 35 & 198 & 0 & 7.9 & 1.30 \\
\hline 15 & Pieroni and Giusti, 2009 & Upper Varaita Valley/ Piedmont & 88 & 0 & 3 & 3 & 3.4 & 85 & 198 & 0 & 3.4 & 1.07 \\
\hline 16 & $\begin{array}{l}\text { Pradhan and Badola, } \\
2008 \\
\end{array}$ & $\begin{array}{c}\text { Dzongu Valley/ North Sikkim, } \\
\text { India }\end{array}$ & 118 & 3 & 4 & 7 & 5.9 & 111 & 194 & 2.5 & 3.4 & 2.34 \\
\hline 17 & Shah and Rahim, 2017 & Soon Valley/ Khushab, Pakistan & 70 & 11 & 17 & 28 & 40 & 42 & 173 & 15.7 & 24.3 & 14.9 \\
\hline 18 & Tetali et al., 2009 & Parinche Valley/ Maharashtra, India & 28 & 0 & 5 & 5 & 17.8 & 23 & 196 & 0 & 17.8 & 2.34 \\
\hline 19 & Ali and Qaiser, 2009 & Chitral Valley/ Pakistan & 83 & 4 & 6 & 10 & 12.04 & 73 & 191 & 4.8 & 7.2 & 3.9 \\
\hline 20 & Rasingam, 2012 & Pillur Valley/ Tamil Nadu, India & 74 & 0 & 12 & 12 & 16.2 & 62 & 189 & 0 & 16.2 & 5.02 \\
\hline 21 & Sanz-Biset et al., 2009 & Chazuta Valley/ Peruvian Amazon & 289 & 1 & 0 & 1 & 0.3 & 288 & 200 & 0.3 & 0 & 0.20 \\
\hline 22 & Sharma et al., 2004 & $\begin{array}{c}\text { Parvati Valley/ western Himalaya, } \\
\text { India }\end{array}$ & 50 & 4 & 3 & 7 & 14 & 43 & 194 & 8 & 6 & 3.04 \\
\hline 23 & $\begin{array}{l}\text { Teklehaymanot and } \\
\text { Giday, } 2010\end{array}$ & lower Omo River Valley/ Ethiopia & 57 & 2 & 4 & 6 & 10.5 & 51 & 195 & 3.5 & 7.01 & 2.5 \\
\hline
\end{tabular}

APPLIED ECOLOGY AND ENVIRONMENTAL RESEARCH 17(2):4725-4805.

http://www.aloki.hu • ISSN 15891623 (Print) • ISSN 17850037 (Online)

DOI: http://dx.doi.org/10.15666/aeer/1702_47254805

(c) 2019, ALÖKI Kft., Budapest, Hungary 
The high numbers of species recorded in the present work were wild (92.6\%). In the current study, the most frequent plant part used ethnobotanically by the local people was flowers $(35 \%)$, followed by leaves $(18 \%)$, whole plant $(12 \%)$, pods $(10 \%)$, twigs $(8 \%)$, aerial parts $(8 \%)$, rhizome and seeds (3\% each), stem (1.6\%) and fruit (1\%) (Fig. 3). A total of 261 preparations were recorded and the most preferred method of preparation was decoction $(41 \%)$, followed by infusion (18\%), raw form $(14 \%)$, paste $(8 \%)$, juice $(5 \%)$, cooked form (3\%), latex, smoke, poultice and powder ( $2 \%$ each), oil, ash and extract (1\% each) (Fig. 4).

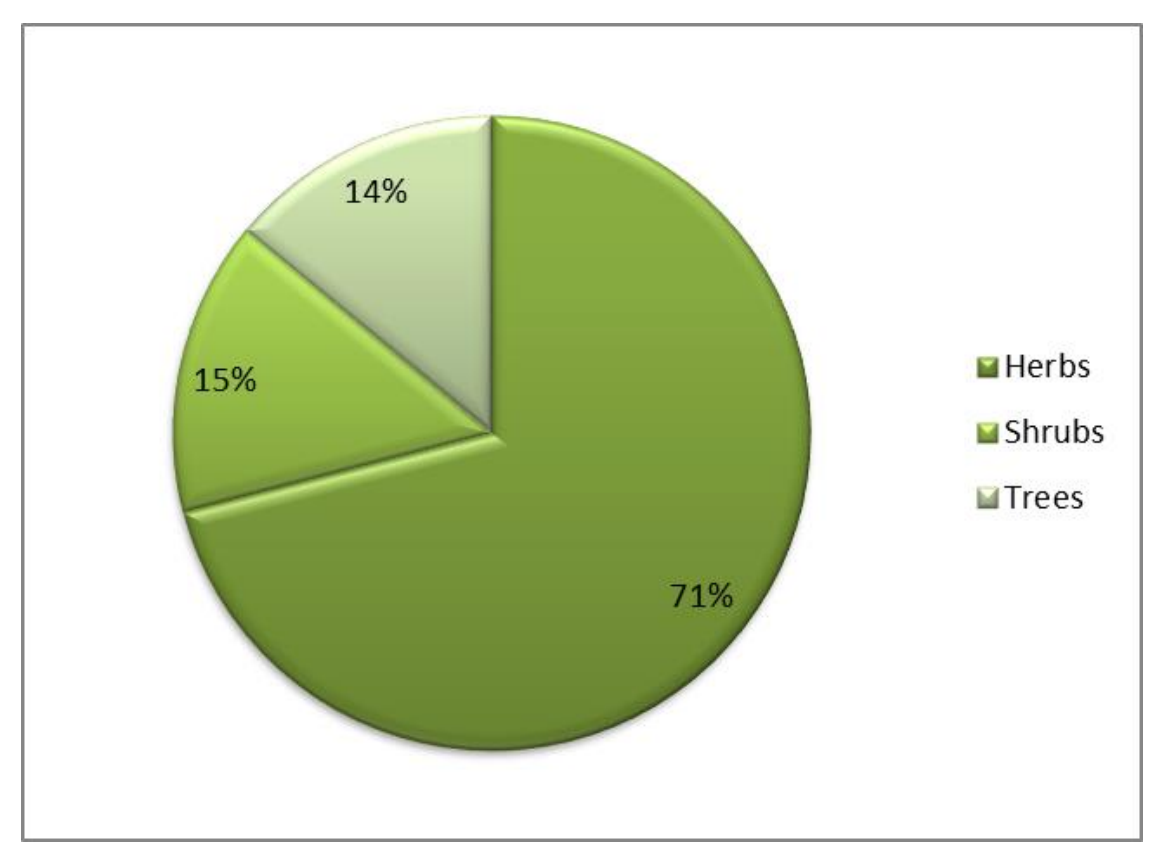

Figure 2. Life form of reported medicinal plants

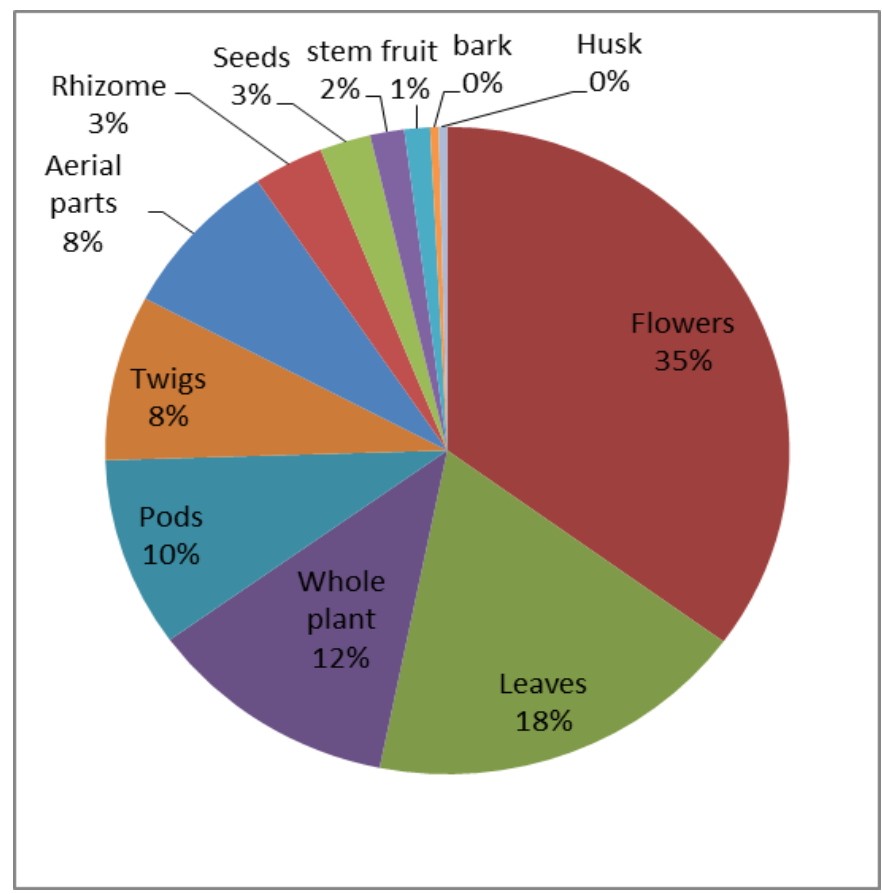

Figure 3. Percentage of part used of plant species 


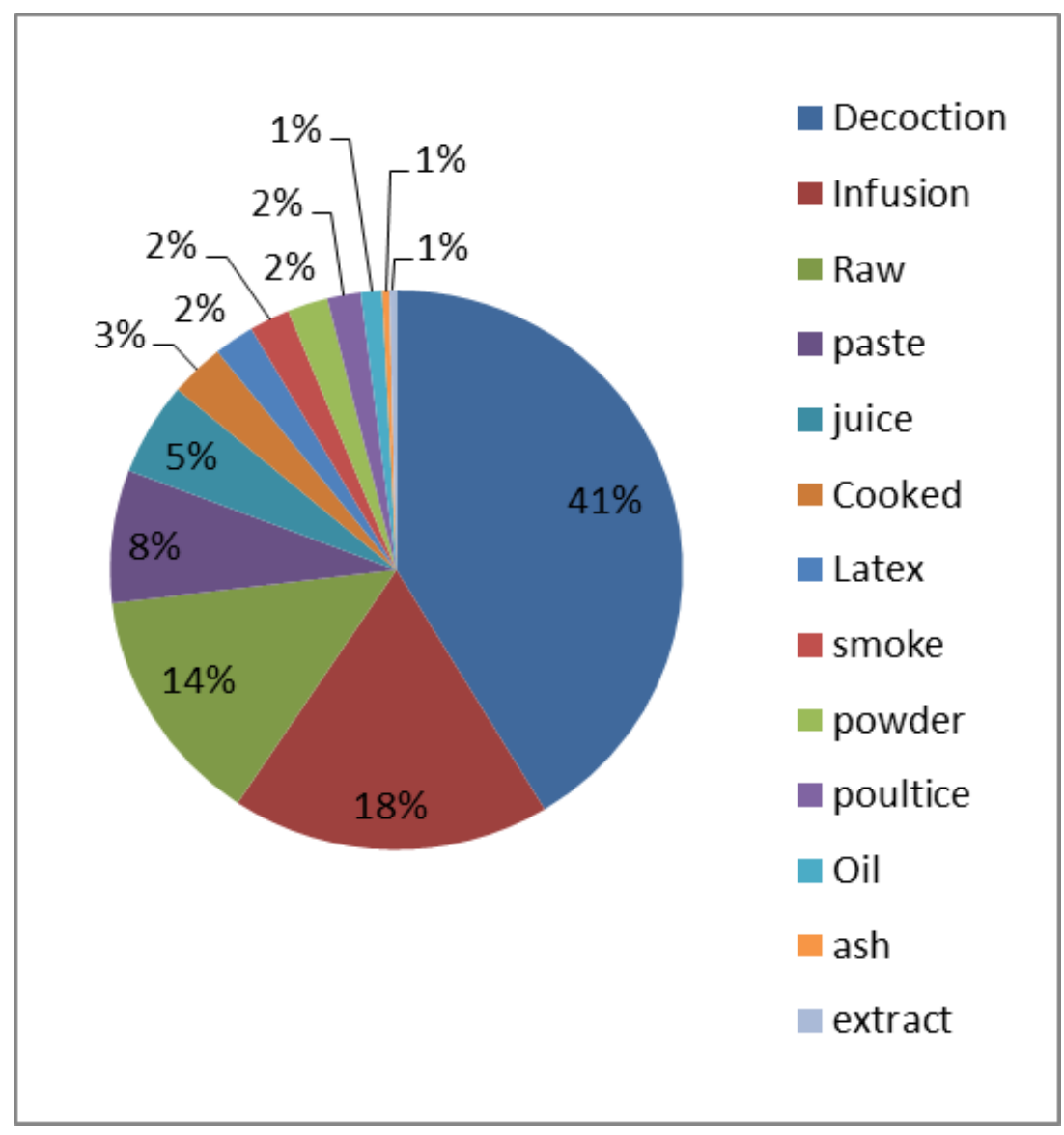

Figure 4. Percentage of mode of utilization

\section{Discussion}

Ethnobotanical knowledge plays an important role in exploring the human-plant relationship and medicinal uses of native flora. In this study possession of traditional knowledge about medicinal plants was found among the people with low level of education and among the age group of $46-<65$. This is because inhabitants of the study area are mostly associated with raring of animals and other depends on available land for farming, they have least concern about acquiring higher education. But due to intimate relation with nature they have enough knowledge about the use of these plants. On the other hand, the informants who are educated are unaware from the uses of plants because of least interaction with the nature.

Highest use report of plants belonging to Fabaceae and Lamiaceae is due to their dominance and wider occurrence as easily available plants are likely to be used frequently by local inhabitant (Agelet and Valles, 2001; Johns et al., 1990) Similar findingis reported by Cornara et al., (2009); Giday et al., 2009; Saslis-Lagoudakis et al., 2011; Teklehaymanot, 2009). Moreover, the ascendancy of these families reveals the presence of a wide range of bioactive compounds in respective taxa belonging to these families. The diversity of bioactive compounds and secondary metabolites may be the reason for the multi-species use one or more health problems, as a single compound may not be the reason of efficacy (Verpoorte et al., 2005). The Poaceae had less aforementioned proceedings in ethno botanical investigations (Cakilcioglu and Turkoglu, 2010; Kadir et al., 2012; Rokaya et al., 2010) conducted at global level. In present study the reason of frequent use of family Poaceae is mainly the dependency of 
locals for fuel (e.g., Saccharum bengalense, S. revennae, S. spontaneum etc.), thatching (Arundo donax, Phragmites australis etc.), forage (Cymbopogon commutatus, Cynodon dactylon, Cenchrus echinatus, Chloris gayana, Coix lacryma-jobi, Dactyloctenium aegyptium, Desmostachya bipinnata, Dichanthium annulatum etc.) and other ethnobotanical uses (medicinal) as compared to medicinal values, because lush herbaceous plants are abundant in study area. Such findings indicate great reliance of inhabitants on a diversity of plant species to treat ailments, and represent a good sign of the intense facts on plant based treatments (Nadembega et al., 2011). Among the reported plant species, some medicinal plants were of high indigenous priority thus are commonly cited by majority of the respondents. The highly cited medicinal plant species those having at least 32 citations or more (Table 1) were Aloe vera, Calotropis procera, Mentha longifolia, Tamarix dioica, Ziziphus nummularia, Nerium oleander, Salvadora persica, Ziziphus mauritiana, Tamarix aphylla, Typha angustata, T. latifolia, Peganum harmala, Tribulus terrestris, Acacia modesta, Albizia procera, Eruca sativa, Ricinus communis, Withania coagulans, Withania somnifera, Morus alba, Conyza canadensis, Ocimum basilicum, Rumex dentatus and Urtica dioica. These plants may reported frequently due to their abundance in the area and there is prevailing beliefs among the inhabitants to consider these plants medicinally effective, as majority of informants stated that maximum treatment were cured at domestic level because of unavailability of healthcare services and high cost of synthetic medications. The reason of frequent citation of these plants may be the presence of valuable and pharmacologically active bioactive compounds. Favorable climatic and topographic conditions of the study area might have positive contribution toward the rapid adaptation of herbaceous flora as compared to other life forms (Ayyanar and Ignacimuthu, 2011; Giday et al., 2003; Mesfin et al., 2012; Uniyal et al., 2006). According to most of the informants the fast restoration and higher potency of herbs is the main reason to have higher preferences for medicinal purposes and herbs are ephemeral in habit. Similar results were also discussed in previous documentations (Megersa et al., 2013; Upadhyay et al., 2010; Upadhyay et al., 2007). Herbs are rich in bioactive compounds (Srithi et al., 2009). The major use of flower is unique to this study when, compared with other ethnomedicinal studies (Rahman et al., 2016; Shah and Rahim, 2017) conducted in Pakistan. Local people believe that collecting a plant in flowering period is very important for the effectiveness of the formulation. This may be due to the richness of essential oils or the existence of bioactive compounds in flowers as compared to other parts. However, collection of premature flower and its excessive use may have negative impacts on plants sustainability. Furthermore, over-exploitation of seeds and roots for local uses can lead to a drastic decline in the populations of medicinal plants (Ghimire et al., 2008) and consequently an expatriation of medicinal species from the area. Therapeutic uses of leaves are defensible (Giday et al., 2003; Zheng and Xing, 2009) which is reported as the second highest part used in this study. Use of leaves in traditional medication is a common practice which is also reported in several other studies (Asase et al., 2010; Asase and Oppong-Mensah, 2009; Koudouvo et al., 2011; Nadembega et al., 2011; Nguta et al., 2010). The use of leaves may be of high preference due to its photosynthetic function and presence of secondary metabolites for defense from herbivores which could be medicinally important for curing various ailments in humans (Bhattarai et al., 2006; Ghorbani, 2005). In addition, leaf collection is ecologically sustainable and is easy to collect as compared to other plant parts (Giday et al., 2009).

Boiling the dried and stored plant parts for herbal preparation is considered more effective as compared to other methods. It is a general observation that heating process 
speed up several biological reactions ensuring the extraction of countless vigorous compound (Al-Adhroey et al., 2010; Chen et al., 2008; Han et al., 2007; Zhang et al., 2005). Second rated mode of utilization reported in the area was infusion. Fresh and healthy plant parts are used for making infusions. Local people believe that infusion is one of the highly effective recipe which is easy to prepare and several effective compounds that plays a crucial role in the medication will not degraded during the process of preparation (Dike et al., 2012; Idowu et al., 2010). A handful amount (quantity that can be held or griped in a hand) of a patient is considered as an exact dose for medication from generation to generation. In the study area this is the most common way to measure a dose of drug required for different age groups from children to adults. Doses are taken twice or thrice a day depending upon the condition of the disease treated. Water was main and common solvent used in herbal remedies (Andrade-Cetto, 2009; Lee et al., 2008; Poonam and Singh, 2009; Prashanth et al., 2001; Ssegawa and Kasenene, 2007) since most of them were prepared as decoction or infusion. This practice is highly convenient because water is easily accessible, the easiest vehicle for herbal preparation and also expected not to disturb the chemical composition of the active constituents (Nunkoo and Mahomoodally, 2012). Some other ingredients sugar, salt, honey, olive oil, mustard oil and lemon are also added to improve the taste and to have additional effective that can help to reduce nausea, vomiting and constipation.

\section{Quantitative analysis of ethnobotanical data}

\section{Use value (UV), Frequency of citation $(F C)$ and Relative frequency citation $(R F C)$}

Use value implies the relative significance of plant species on the basis of number of informants (FC) who mention the uses reported for respective plant species. The plant species with high UV (Eq.l) are suggested for further pharmacological and phytochemical screening to develop new drugs.

Relative frequency citation (RFC) (Eq.2) is a quantitative index that estimates the local reputation of every plant species used by the ethnic folks (Vitalini et al., 2013). It is calculated from Frequency of Citation (FC) divided by the total number of informants (N) who participated in the survey (Tardío and Pardo-de-Santayana, 2008) ( Table 1).

The high RFC value suggests that these plants were known to majority of the informants. The high values of RFC help to identify species for which an appropriate conservation and sustainable utilization strategies should be formulated to ensure regular supply of raw materials for the future (Asase et al., 2005).

\section{Comparison of different indices}

Informant consensus factor (ICF) (Eq.3) values of reported medicinal plants were calculated for the categorized ailments (Table 1). Fifteen primary ailment categories were identified: Table 3. ICF values range from 0 to 0.7 (Table 3). Highest ICF value (0.7) was reported for oral and dental problems group, followed by respiratory problems (0.4), gastrointestinal problems, antidote and skin problems ( 0.3 each). The reason of high ICF could be the common happening of these illnesses in the community due to poor sanitation practice, low economic status and lack of adequate modern health care of in the Valley (Bieski et al., 2015). High ICF values undoubtedly disclose a noteworthy number of reports on the use of these taxa for a group of health problems (Baydoun et al., 2015). The slightest agreement among the informants was detected for plants used for group of sexual disorders, ear, nose and throat problems (ENT), eye ailments and other diseases which include pain, small pox, mumps, insect repellent, antitumor and anti-allergic, all these groups containing the zero ICF value. The least 
value of ICF for ear, nose and throat problems (ENT) and eye diseases is in accordance with other studies (Bibi et al., 2015; Jamila and Mostafa, 2014). These low ICF values could be credited to the trend of folks in native or urban societies to use conventional medicines for curing predictable diseases, even in current times (Upadhyay et al., 2011).

Table 3. ICF Value of medicinal plants used for treatment of various diseases (ICF*=Informant Consensus Factor)

\begin{tabular}{c|c|c|c}
\hline Disease category & No. of use reports & No. of taxa & ICF* \\
\hline Respiratory problems & 78 & 49 & 0.4 \\
Urinogenital diseases & 9 & 7 & 0.25 \\
Gastrointestinal problems & 145 & 99 & 0.3 \\
Musculoskeletal disorders & 56 & 46 & 0.2 \\
Sexual disorders & 53 & 53 & 0 \\
Skin problems & 83 & 61 & 0.3 \\
Glandular disorders & 29 & 16 & 0.03 \\
Antidote & 22 & 46 & 0.3 \\
Cardiovascular diseases & 51 & 18 & 0.1 \\
Neurological disorders & 20 & 13 & 0.1 \\
ENT problems & 13 & 3 & 0 \\
Eye ailments & 3 & 55 & 0 \\
Fever & 68 & 7 & 0.2 \\
Oral and Dental problems & 19 & 5 & 0.7 \\
Hair problems & 6 & 10 & 0.2 \\
Others & 10 &
\end{tabular}

\section{Comparative analysis of documented data with previous literature}

To conclude whether the usage of plants by communities of Namal Valley were previously reported or not for medicinal value, a literature study on ethnobotanical use was done and comparative results are listed in Table 1. Twenty three research papers from different valleys were selected to compare the data and it was observed that various plants have novel use reports and some plant species like Viola cinerea, Pulicaria glutinosa and Pseudogaillonia hymnostephana from the study area are reported for the first time and previously with no or rare use reports. In this study a total of 217 plant species were documented. Literature review of described medicinal plants have shown that the similarity of uses varies from $0 \%$ to $15.7 \%$, while dissimilar uses range up to $32.5 \%$ (Table 2) (Eq.4). In our study high degree of similarity was found with studies directed in Bana Valley in Kotli, Pakistan by Amjad. (2015) and Soon Valley in Khushab, Pakistan by Shah and Rahim. (2017) with JI 23.9 and 14.9 respectively (Table 2). The cause of maximum similarity index may be the resemblance in plant diversity and their multipurpose uses against numerous health related problems in the study area. Noteworthy multiethnic discrepancy may be the cause of deviation in JI that are topmost symbolic of variation in habitation and populace (Leonti et al., 2009). The resemblance and inconsistency in ethnomedicinal studies appear to point out the significance of tribal wakefulness of medicinal plants in miscellaneous regions where chronological (Moerman, 1998) environmental (Ladio et al., 2007), phytochemical and organoleptic (Leonti et al., 2003) structures add in their assortment. 


\section{Limitations of the study, insights gained and approaches for improvements}

This study was primarily aimed to document indigenous ethnobotanical knowledge of native communities of Namal Valley and evaluates their reliability within Pakistan and globally. However, few limitations still exists regarding sufficient interpretation of data. For example the native people have no acquaintance of common names/ vernacular names of some plant and similarly no report about their ethnomedicinal value and their use is confined to fuel, forage, thatching etc. Another obstruction of the study was that it did not investigate into the danger of invasive species and, documentation and dispersal of the introduced species.

\section{Side effects of medicinal and other plants}

In this study the documented ethnobotanical data generally elaborate the medicinal usage of the indigenous plant species, but it was observed that informants were much careful in using some of the plants such as Achyranthes aspera, Calotropis procera, Cannabis sativa, Croton bonplandianum, Cuscuta reflexa, Datura metel, Dodonaea viscosa, Euphorbia helioscopia, E. heterophylla, E. hirta, E. peplus, E. prostrata, E. serpens, Heliotropium currasavicum, H. europaeum, Ipomoea carnea, Lantana camara, Lathyrus aphaca, Nerium oleander, Ricinus communis, Rhazya stricta, Solanum elaeagnifolium, S. xanthocarpum, S. nigrum, S. surattense, S. incanum. The inhabitants are fully aware about the fact that these plants can cause symptom of toxicity including abortion, restlessness, depression, skin inflammation, vomiting, abdominal pain, nausea, impotency, sterility, dizziness and hallucination. People have a general knowledge about toxic signs and their interpretation of toxicity is based on the observation from generation to generation. Foremost taxonomic classification level for assessing the efficacy of plant to native societies is family (Thomas et al., 2009). Same is factual for the toxicity of plants (Huai et al., 2010). Fabaceae, Asteraceae, Euphorbiaceae and Apocynaceae have been described as the chief families comprising toxic plants in various studies (Levetin and McMahon, 2008; Huai et al., 2010; Ozturk et al., 2008). The reason of toxicity of these families is the presence of different toxins such as alkaloids, dicoumarin, glycosides, photosensitizing compounds, saponin, selenium (Fabaceae), acrid substances, alcohol, alkaloids, glycosides, nitrogenous compounds, photosensitizing compounds, saponins, selenium, volatile oils (Asteraceae), acrid substances, croton oil, photosensitizing compounds, biterpinoids, triterpenoids, steroids, alkaloids, cyanogenic glycosides and glucosinolates (Euphorbiaceae) and resin, glycosides (Apocynaceae) (Barla et al., 2006; Madureira et al., 2004; RIZK, 1987; Yamamura et al., 1989; Zhang and Guo, 2006). Further study of herbal toxicity in human is needed to certify safety and boost patient confidence about herbal remedies.

\section{Conclusions and recommendations}

This study exposed that traditional medication, mainly comprising the practice of medicinal plants playing a substantial role in curing the basic healthcare necessities of the inhabitants of Namal Valley, Pakistan. Usage of traditional medicine as a dominant part of their beliefs, inadequate access to recent health care conveniences and the cheerful prosperity of natural assets could be deliberated as the key influences for the prolongation of these traditional practices. The instant and thoughtful hazard to the native medicinal flora in the study area appears from the growing impact of overharvesting, overgrazing and deforestation due to human activities. There is dire necessity for thoughtful exertions to create awareness in the native people so that the 
proper actions should be taken to protect the suitable surroundings/habitations mandatory to defend and conserve the medicinal plants in their natural ecosystems. Because of lack of written documents of conservation of plants and habitats, researches like this study is compulsory to protect primeval retentions, to encourage the flow of information to the younger generations, to preserve ethno-biodiversity and to deliver a beginning point for further pharmacological, biological and chemical investigations on medicinal objects.

Acknowledgements. The authors appreciatively thank all the guides the traditional healers who accepted to participate to the study. The study was supported by Higher Education Commission Pakistan (Programme No. 20-1599/ 09/3007).

\section{REFERENCES}

[1] Abu-Irmaileh, B. E., Afifi, F. U. (2003): Herbal medicine in Jordan with special emphasis on commonly used herbs. - Journal of Ethnopharmacology 89(2-3): 193-197.

[2] Agelet, A., Valles, J. (2001): Studies on pharmaceutical ethnobotany in the region of Pallars (Pyrenees, Catalonia, Iberian Peninsula) Part I. General results and new or very rare medicinal plants. - Journal of Ethnopharmacology 77(1): 57-70.

[3] Ahmad, M., Sultana, S., Fazl-i-Hadi, S., Ben Hadda, T., Rashid, S., Zafar, M., Khan, M. A., Khan, M. P. Z., Yaseen, G. (2014): An Ethnobotanical study of Medicinal Plants in high mountainous region of Chail valley (District Swat-Pakistan). - Journal of ethnobiology and ethnomedicine 10(1): 36.

[4] Ahmad, S. S., Husain, S. Z. (2008): Ethno medicinal survey of plants from salt range (Kallar Kahar) of Pakistan. - Pakistan Journal of Botany 40(3): 1005-1011.

[5] Akerele, O. (1988): Medicinal plants and primary health care: an agenda for action. Fitoterapia 59(5): 355-363.

[6] Al-Adhroey, A. H., Nor, Z. M., Al-Mekhlafi, H. M., Mahmud, R. (2010): Ethnobotanical study on some Malaysian anti-malarial plants: A community based survey. - Journal of ethnopharmacology 132(1): 362-364.

[7] Ali, H., Qaiser, M. (2009): The ethnobotany of Chitral valley, Pakistan with particular reference to medicinal plants. - Pakistan Journal of Botany 41(4): 2009-2041.

[8] Amjad, M. S. (2015): Ethnobotanical profiling and floristic diversity of Bana Valley, Kotli (Azad Jammu and Kashmir), Pakistan. - Asian Pacific Journal of Tropical Biomedicine 5(4): 292-299.

[9] Andrade-Cetto, A. (2009): Ethnobotanical study of the medicinal plants from Tlanchinol, Hidalgo, México. - Journal of ethnopharmacology 122(1): 163-171.

[10] Asase, A., Akwetey, G. A., Achel, D. G. (2010): Ethnopharmacological use of herbal remedies for the treatment of malaria in the Dangme West District of Ghana. - Journal of ethnopharmacology 129(3): 367-376.

[11] Asase, A., Oppong-Mensah, G. (2009): Traditional antimalarial phytotherapy remedies in herbal markets in southern Ghana. - Journal of ethnopharmacology 126(3): 492-499.

[12] Asase, A., Oteng-Yeboah, A. A., Odamtten, G. T., Simmonds, M. S. (2005): Ethnobotanical study of some Ghanaian anti-malarial plants. - Journal of Ethnopharmacology 99(2): 273-279.

[13] Ayyanar, M., Ignacimuthu, S. (2011): Ethnobotanical survey of medicinal plants commonly used by Kani tribals in Tirunelveli hills of Western Ghats, India. - Journal of Ethnopharmacology 134(3): 851-864.

[14] Balunas, M. J., Kinghorn, A. D. (2005): Drug discovery from medicinal plants. - Life sciences 78(5): 431-441.

[15] Bano, A., Ahmad, M., Hadda, T. B., Saboor, A., Sultana, S., Zafar, M., Khan, M. P. Z., Arshad, M., Ashraf, M. A. (2014): Quantitative ethnomedicinal study of plants used in 
the skardu valley at high altitude of Karakoram-Himalayan range, Pakistan. - Journal of ethnobiology and ethnomedicine 10(1): 43.

[16] Barla, A., Bİrman, H., Kültür, Ş., Öksüz, S. (2006): Secondary metabolites from Euphorbia helioscopia and their vasodepressor activity. - Turkish Journal of Chemistry 30(3): 325-332.

[17] Baydoun, S., Chalak, L., Dalleh, H., Arnold, N. (2015): Ethnopharmacological survey of medicinal plants used in traditional medicine by the communities of Mount Hermon, Lebanon. - Journal of ethnopharmacology 173: 139-156.

[18] Belayneh, A., Asfaw, Z., Demissew, S., Bussa, N. F. (2012): Medicinal plants potential and use by pastoral and agro-pastoral communities in Erer Valley of Babile Wereda, Eastern Ethiopia. - Journal of Ethnobiology and Ethnomedicine 8(1): 42.

[19] Bhattarai, S., Chaudhary, R. P., Taylor, R. S. (2006): Ethnomedicinal plants used by the people of Manang district, central Nepal. - Journal of Ethnobiology and Ethnomedicine 2(1): 41.

[20] Bibi, T., Ahmad, M., Tareen, N.M., Jabeen, R., Sultana, S., Zafar, M., Zain-ul-Abidin, S. (2015): The endemic medicinal plants of Northern Balochistan, Pakistan and their uses in traditional medicine. - Journal of ethnopharmacology 173: 1-10.

[21] Bieski, I. G. C., Leonti, M., Arnason, J. T., Ferrier, J., Rapinski, M., Violante, I. M. P., Balogun, S. O., Pereira, J. F. C. A., Figueiredo, R. D. C. F., Lopes, C. R. A. S. (2015): Ethnobotanical study of medicinal plants by population of valley of Juruena region, legal Amazon, Mato Grosso, Brazil. - Journal of ethnopharmacology 173: 383-423.

[22] Bulut, G., Haznedaroğlu, M. Z., Doğan, A., Koyu, H., Tuzlac1, E. (2017): An ethnobotanical study of medicinal plants in Acipayam (Denizli-Turkey). - Journal of herbal medicine 10: 64-81.

[23] Cakilcioglu, U., Turkoglu, I. (2010): An ethnobotanical survey of medicinal plants in Sivrice (Elazığ-Turkey). - Journal of Ethnopharmacology 132(1): 165-175.

[24] Canales, M., Hernández, T., Caballero, J., De Vivar, A. R., Avila, G., Duran, A., Lira, R. (2005): Informant consensus factor and antibacterial activity of the medicinal plants used by the people of San Rafael Coxcatlán, Puebla, México. - Journal of Ethnopharmacology 97(3): 429-439.

[25] Chen, G., Yang, M., Song, Y., Lu, Z., Zhang, J., Huang, H., Guan, S., Wu, L., Guo, D. A. (2008): Comparative analysis on microbial and rat metabolism of ginsenoside Rb1 by high-performance liquid chromatography coupled with tandem mass spectrometry. Biomedical Chromatography 22(7): 779-785.

[26] Cornara, L., La Rocca, A., Marsili, S., Mariotti, M. (2009): Traditional uses of plants in the Eastern Riviera (Liguria, Italy). - Journal of Ethnopharmacology 125(1): 16-30.

[27] Dabheliya, J., Khan, S., Joshipura, M., Vasoya, M., Patel, S., Vijaya, S. (2010): Diuretic potential of aqueous extract of fruits of Withania coagulans Dunal in experimental rats. International journal of pharmacy and pharmaceutical sciences 2(4): 51-53.

[28] Dike, I. P., Obembe, O. O., Adebiyi, F. E. (2012): Ethnobotanical survey for potential anti-malarial plants in south-western Nigeria. - Journal of ethnopharmacology 144(3): 618-626.

[29] Fortini, P., Di Marzio, P., Guarrera, P. M., Iorizzi, M. (2016): Ethnobotanical study on the medicinal plants in the Mainarde Mountains (central-southern Apennine, Italy). Journal of ethnopharmacology 184: 208-218.

[30] Gazzaneo, L. R. S., De Lucena, R. F. P., de Albuquerque, U. P. (2005): Knowledge and use of medicinal plants by local specialists in an region of Atlantic Forest in the state of Pernambuco (Northeastern Brazil). - Journal of Ethnobiology and Ethnomedicine 1(1): 9.

[31] Ghimire, S. K., Gimenez, O., Pradel, R., McKey, D., Aumeeruddy-Thomas, Y. (2008): Demographic variation and population viability in a threatened Himalayan medicinal and aromatic herb Nardostachys grandiflora: matrix modelling of harvesting effects in two contrasting habitats. - Journal of Applied Ecology 45(1): 41-51.

[32] Ghorbani, A. (2005): Studies on pharmaceutical ethnobotany in the region of Turkmen Sahra, north of Iran: (Part 1): General results. - Journal of ethnopharmacology 102(1): 58-68. 
[33] Giday, M., Asfaw, Z., Elmqvist, T., Woldu, Z. (2003): An ethnobotanical study of medicinal plants used by the Zay people in Ethiopia. - Journal of Ethnopharmacology 85(1): 43-52.

[34] Giday, M., Asfaw, Z., Woldu, Z. (2009): Medicinal plants of the Meinit ethnic group of Ethiopia: an ethnobotanical study. - Journal of Ethnopharmacology 124(3): 513-521.

[35] González-Tejero, M., Casares-Porcel, M., Sánchez-Rojas, C., Ramiro-Gutiérrez, J., Molero-Mesa, J., Pieroni, A., Giusti, M., Censorii, E., De Pasquale, C., Della, A. (2008): Medicinal plants in the Mediterranean area: synthesis of the results of the project Rubia. Journal of Ethnopharmacology 116(2): 341-357.

[36] Han, J., Ye, M., Guo, H., Yang, M., Wang, B-R., Guo, D-A. (2007): Analysis of multiple constituents in a Chinese herbal preparation Shuang-Huang-Lian oral liquid by HPLCDAD-ESI-MSn. - Journal of Pharmaceutical and Biomedical Analysis 44(2): 430-438.

[37] Haq, F. (2012): The ethno botanical uses of medicinal plants of Allai Valley, Western Himalaya Pakistan. - International Journal of Plant Research 2(1): 21-34.

[38] Hazrat, A., Nisar, M., Shah, J., Ahmad, S. (2011): Ethnobotanical study of some elite plants belonging to Dir, Kohistan valley, Khyber Pukhtunkhwa, Pakistan. - Pakistan Journal of Botany 43(2): 787-795.

[39] Heinrich, M. (2000): Ethnobotany and its role in drug development. - Phytotherapy Research 14(7): 479-488.

[40] Heinrich, M., Ankli, A., Frei, B., Weimann, C., Sticher, O. (1998): Medicinal plants in Mexico: Healers' consensus and cultural importance. - Social Science \& Medicine 47(11): 1859-1871.

[41] Heinrich, M., Edwards, S., Moerman, D.E., Leonti, M. (2009): Ethnopharmacological field studies: A critical assessment of their conceptual basis and methods. - Journal of Ethnopharmacology 124(1): 1-17.

[42] Heinrich, M., Gibbons, S. (2001): Ethnopharmacology in drug discovery: an analysis of its role and potential contribution. - Journal of Pharmacy and Pharmacology 53(4): 425-432.

[43] Huai, H., Dong, Q., Liu, A. (2010): Ethnomedicinal analysis of toxic plants from five ethnic groups in China. - Ethnobotany Research and Applications 8: 169-179.

[44] Idowu, O., Soniran, O., Ajana, O., Aworinde, D. (2010): Ethnobotanical survey of antimalarial plants used in Ogun State, Southwest Nigeria. - African Journal of Pharmacy and Pharmacology 4(2): 055-060.

[45] Jamila, F., Mostafa, E. (2014): Ethnobotanical survey of medicinal plants used by people in Oriental Morocco to manage various ailments. - Journal of ethnopharmacology 154(1): 76-87.

[46] Jiofack, T., Fokunang, C., Guedje, N., Kemeuze, V. (2009): Ethnobotany and phytomedicine of the upper Nyong valley forest in Cameroon. - African Journal of Pharmacy and Pharmacology 3(4): 144-150.

[47] Johns, T., Kokwaro, J. O., Kimanani, E. K. (1990): Herbal remedies of the Luo of Siaya District, Kenya: establishing quantitative criteria for consensus. - Economic Botany 44(3): 369-381.

[48] Kadir, M. F., Sayeed, M. S. B., Shams, T., Mia, M. (2012): Ethnobotanical survey of medicinal plants used by Bangladeshi traditional health practitioners in the management of diabetes mellitus. - Journal of Ethnopharmacology 144(3): 605-611.

[49] Khan, M. A., Khan, M. A., Hussain, M., Ghulam, G. M. (2010): An ethnobotanical inventory of Himalayan region Poonch valley Azad Kashmir (Pakistan). - Ethnobotany Research and Applications 8: 107-123.

[50] Khan, S. M., Page, S., Ahmad, H., Shaheen, H., Ullah, Z., Ahmad, M., Harper, D. M. (2013): Medicinal flora and ethnoecological knowledge in the Naran Valley, Western Himalaya, Pakistan. - Journal of ethnobiology and ethnomedicine 9(1): 4.

[51] Khan, S. W., Khatoon, S. (2008): Ethnobotanical studies on some useful herbs of Haramosh and Bugrote valleys in Gilgit, northern areas of Pakistan. - Pakistan Journal of Botany 40(1): 43.

[52] Kloutsos, G., Balatsouras, D. G., Kaberos, A. C., Kandiloros, D., Ferekidis, E., Economou, C. (2001): Upper airway edema resulting from use of Ecballium elaterium. The Laryngoscope 111(9): 1652-1655. 
[53] Koudouvo, K., Karou, D., Kokou, K., Essien, K., Aklikokou, K., Glitho, I., Simpore, J., Sanogo, R., De Souza, C., Gbeassor, M., (2011): An ethnobotanical study of antimalarial plants in Togo Maritime Region. - Journal of ethnopharmacology 134(1): 183-190.

[54] Kunwar, R. M., Acharya, R. P., Chowdhary, C. L., Bussmann, R. W. (2015): Medicinal plant dynamics in indigenous medicines in farwest Nepal. - Journal of ethnopharmacology 163: 210-219.

[55] Ladio, A., Lozada, M., Weigandt, M. (2007): Comparison of traditional wild plant knowledge between aboriginal communities inhabiting arid and forest environments in Patagonia, Argentina. - Journal of arid environments 69(4): 695-715.

[56] Lee, S., Xiao, C., Pei, S. (2008): Ethnobotanical survey of medicinal plants at periodic markets of Honghe Prefecture in Yunnan Province, SW China. - Journal of Ethnopharmacology 117(2): 362-377.

[57] Leonti, M., Casu, L., Sanna, F., Bonsignore, L. (2009): A comparison of medicinal plant use in Sardinia and Sicily-De Materia Medica revisited? - Journal of ethnopharmacology 121(2): 255-267.

[58] Leonti, M., Sticher, O., Heinrich, M. (2003): Antiquity of medicinal plant usage in two Macro-Mayan ethnic groups (Mexico) - Journal of ethnopharmacology 88(2-3): 119-124.

[59] Levetin, E., McMahon, K., (2008): Plants and Society, 5th edition McGraw-Hill, New York.

[60] Madureira, A. M., Ferreira, M-J. U., Gyemant, N., Ugocsai, K., Ascenso, J. R., Abreu, P. M., Hohmann, J., Molnár, J. (2004): Rearranged jatrophane-type diterpenes from euphorbia species. Evaluation of their effects on the reversal of multidrug resistance. Planta medica 70(1): 45-49.

[61] Martin, G. (1985): Ethnobotany: a methods manual Chapman and hall. - New York, EE. UU.

[62] Megersa, M., Asfaw, Z., Kelbessa, E., Beyene, A., Woldeab, B. (2013): An ethnobotanical study of medicinal plants in Wayu Tuka district, east Welega zone of oromia regional state, West Ethiopia. - Journal of ethnobiology and ethnomedicine 9(1): 68.

[63] Menale, B., De Castro, O., Cascone, C., Muoio, R. (2016): Ethnobotanical investigation on medicinal plants in the Vesuvio National Park (Campania, southern Italy). - Journal of ethnopharmacology 192: 320-349.

[64] Mesfin, A., Giday, M., Animut, A., Teklehaymanot, T. (2012): Ethnobotanical study of antimalarial plants in Shinile District, Somali Region, Ethiopia, and in vivo evaluation of selected ones against Plasmodium berghei. - Journal of Ethnopharmacology 139(1): 221-227.

[65] Mesfin, F., Demissew, S., Teklehaymanot, T. (2009): An ethnobotanical study of medicinal plants in Wonago Woreda, SNNPR, Ethiopia. - Journal of Ethnobiology and Ethnomedicine 5(1): 28.

[66] Moerman, D. (1998): Native American Ethnobotany Timber Press. Oregon. ISBN 088192-453-9.

[67] Nadembega, P., Boussim, J. I., Nikiema, J. B., Poli, F., Antognoni, F. (2011): Medicinal plants in Baskoure, Kourittenga province, Burkina Faso: an ethnobotanical study. Journal of ethnopharmacology 133(2): 378-395.

[68] Nguta, J., Mbaria, J., Gakuya, D., Gathumbi, P., Kiama, S. (2010): Traditional antimalarial phytotherapy remedies used by the South Coast community, Kenya. Journal of ethnopharmacology 131(2): 256-267.

[69] Nunkoo, D. H., Mahomoodally, M. F. (2012): Ethnopharmacological survey of native remedies commonly used against infectious diseases in the tropical island of Mauritius. Journal of ethnopharmacology 143(2): 548-564.

[70] Ozturk, M., Uysal, I., Guecel, S., Mert, T., Akcicek, E., Celik, S. (2008): Ethnoecology of poisonous plants of Turkey and Northern Cyprus. - Pakistan Journal of Botany 40(4): 1359-1386.

[71] Phillips, O., Gentry, A. H., Reynel, C., Wilkin, P., Galvez-Durand, B. (1994): Quantitative ethnobotany and Amazonian conservation. Conservation biology 8(1): 225-248.

[72] Phondani, P. C., Maikhuri, R. K., Rawat, L. S., Farooquee, N. A., Kala, C. P., Vishvakarma, S. R., Saxena, K. G. (2010): Ethnobotanical uses of plants among the 
Bhotiya tribal communities of Niti Valley in Central Himalaya, India. - Ethnobotany Research and Applications 8: 233-244.

[73] Pieroni, A., Giusti, M. E. (2009): Alpine ethnobotany in Italy: traditional knowledge of gastronomic and medicinal plants among the Occitans of the upper Varaita valley, Piedmont. - Journal of Ethnobiology and Ethnomedicine 5(1): 32.

[74] Poonam, K., Singh, G. S. (2009): Ethnobotanical study of medicinal plants used by the Taungya community in Terai Arc Landscape, India. - Journal of ethnopharmacology 123(1): 167-176.

[75] Pradhan, B. K., Badola, H. K. (2008): Ethnomedicinal plant use by Lepcha tribe of Dzongu valley, bordering Khangchendzonga Biosphere Reserve, in north Sikkim, India. Journal of Ethnobiology and Ethnomedicine 4(1): 22.

[76] Prashanth, D., Asha, M., Amit, A., Padmaja, R. (2001): Anthelmintic activity of Butea monosperma. - Fitoterapia 72(4): 421-422.

[77] Rahman, I. U., Ijaz, F., Afzal, A., Iqbal, Z., Ali, N., Khan, S. M. (2016): Contributions to the phytotherapies of digestive disorders: Traditional knowledge and cultural drivers of Manoor Valley, Northern Pakistan. - Journal of ethnopharmacology 192: 30-52.

[78] Rasingam, L. (2012): Ethnobotanical studies on the wild edible plants of Irula tribes of Pillur Valley, Coimbatore district, Tamil Nadu, India. - Asian Pacific Journal of Tropical Biomedicine 2(3): S1493-S1497.

[79] Rehecho, S., Uriarte-Pueyo, I., Calvo, J., Vivas, L.A., Calvo, M.I. (2011): Ethnopharmacological survey of medicinal plants in Nor-Yauyos, a part of the Landscape Reserve Nor-Yauyos-Cochas, Peru. - Journal of ethnopharmacology 133(1): 75-85.

[80] Rizk, A. F. M. (1987): The chemical constituents and economic plants of the Euphorbiaceae. - Botanical Journal of the Linnean Society 94(1-2): 293-326.

[81] Rokaya, M. B., Münzbergová, Z., Timsina, B. (2010): Ethnobotanical study of medicinal plants from the Humla district of western Nepal. - Journal of Ethnopharmacology 130(3): 485-504.

[82] Sanz-Biset, J., Campos-de-la-Cruz, J., Epiquién-Rivera, M. A., Canigueral, S. (2009): A first survey on the medicinal plants of the Chazuta valley (Peruvian Amazon) - Journal of Ethnopharmacology 122(2): 333-362.

[83] Saslis-Lagoudakis, C. H., Williamson, E. M., Savolainen, V., Hawkins, J. A. (2011): Cross-cultural comparison of three medicinal floras and implications for bioprospecting strategies. - Journal of Ethnopharmacology 135(2): 476-487.

[84] Shah, A., Rahim, S. (2017): Ethnomedicinal uses of plants for the treatment of malaria in Soon Valley, Khushab, Pakistan. - Journal of ethnopharmacology 200: 84-106.

[85] Shah, A., Sarvat, R., Shoaib, S., Ayodele, A., Nadeem, M., Qureshi, T., Ishtiaq, M., Abbas, A. (2018): An ethnobotanical survey of medicinal plants used for the treatment of snakebite and scorpion sting among the people of Namal valley, Mianwali district, Punjab, Pakistan. - Applied Ecology and Environmental Research 16(1): 111-143.

[86] Sharma, P. K., Chauhan, N. S., Lal, B. (2004): Observations on the traditional phytotherapy among the inhabitants of Parvati valley in western Himalaya, India. Journal of Ethnopharmacology 92(2-3): 167-176.

[87] Sharma, R., Manhas, R., Magotra, R. (2012): Ethnoveterinary remedies of diseases among milk yielding animals in Kathua, Jammu and Kashmir, India. - Journal of Ethnopharmacology 141(1): 265-272.

[88] Srithi, K., Balslev, H., Wangpakapattanawong, P., Srisanga, P., Trisonthi, C. (2009): Medicinal plant knowledge and its erosion among the Mien (Yao) in northern Thailand. Journal of Ethnopharmacology 123(2): 335-342.

[89] Ssegawa, P., Kasenene, J. M. (2007): Medicinal plant diversity and uses in the Sango bay area, Southern Uganda. - Journal of Ethnopharmacology 113(3): 521-540.

[90] Tabuti, J., Lye, K., Dhillion, S. (2003): Traditional herbal drugs of Bulamogi, Uganda: plants, use and administration. - Journal of Ethnopharmacology 88(1): 19-44.

[91] Tardío, J., Pardo-de-Santayana, M. (2008): Cultural importance indices: a comparative analysis based on the useful wild plants of Southern Cantabria (Northern Spain). Economic Botany 62(1): 24-39. 
[92] Teklehaymanot, T. (2009): Ethnobotanical study of knowledge and medicinal plants use by the people in Dek Island in Ethiopia. - Journal of Ethnopharmacology 124(1): 69-78.

[93] Teklehaymanot, T., Giday, M. (2010): Quantitative ethnobotany of medicinal plants used by Kara and Kwego semi-pastoralist people in lower Omo River Valley, Debub Omo Zone, Southern Nations, Nationalities and Peoples Regional State, Ethiopia. - Journal of Ethnopharmacology 130(1): 76-84.

[94] The Plant List. (2013): Version 1.1. Royal Botanic Garden, Kew, Missouri Botanical Garden, and other collaborators. Published on the Internet; http://www.theplantlist.org/ (accessed 3 April 2014).

[95] Tetali, P., Waghchaure, C., Daswani, P. G., Antia, N. H., Birdi, T. J. (2009): Ethnobotanical survey of antidiarrhoeal plants of Parinche valley, Pune district, Maharashtra, India. - Journal of ethnopharmacology 123(2): 229-236.

[96] Thomas, E., Vandebroek, I., Sanca, S., Van Damme, P. (2009): Cultural significance of medicinal plant families and species among Quechua farmers in Apillapampa, Bolivia. Journal of Ethnopharmacology 122(1), 60-67.

[97] Uniyal, S. K., Singh, K., Jamwal, P., Lal, B. (2006): Traditional use of medicinal plants among the tribal communities of Chhota Bhangal, Western Himalaya. - Journal of ethnobiology and ethnomedicine 2(1): 14.

[98] Upadhyay, B., Dhaker, A. K., Kumar, A. (2010): Ethnomedicinal and ethnopharmacostatistical studies of Eastern Rajasthan, India. - Journal of Ethnopharmacology 129(1): 64-86.

[99] Upadhyay, B., Roy, S., Kumar, A. (2007): Traditional uses of medicinal plants among the rural communities of Churu district in the Thar Desert, India. - Journal of ethnopharmacology 113(3): 387-399.

[100] Upadhyay, B., Singh, K., Kumar, A. (2011): Ethno-veterinary uses and informants consensus factor of medicinal plants of Sariska region, Rajasthan, India. - Journal of Ethnopharmacology 133(1): 14-25.

[101] Verma, S., Singh, S. (2008): Current and future status of herbal medicines. - Veterinary world 1(11): 347-350.

[102] Verpoorte, R., Choi, Y.H., Kim, H.K. (2005): Ethnopharmacology and systems biology: a perfect holistic match. - Journal of Ethnopharmacology 100(1-2): 53-56.

[103] Vitalini, S., Iriti, M., Puricelli, C., Ciuchi, D., Segale, A., Fico, G. (2013): Traditional knowledge on medicinal and food plants used in Val San Giacomo (Sondrio, Italy) -An alpine ethnobotanical study. - Journal of Ethnopharmacology 145(2): 517-529.

[104] Yabesh, J. M., Prabhu, S., Vijayakumar, S. (2014): An ethnobotanical study of medicinal plants used by traditional healers in silent valley of Kerala, India. - Journal of ethnopharmacology 154 (3): 774-789.

[105] Yamamura, S., Shizuri, Y., Kosemura, S., Ohtsuka, J., Tayama, T., Ohba, S., Ito, M., Saito, Y., Terada, Y. (1989): Diterpenes from Euphorbia helioscopia. - Phytochemistry 28(12): 3421-3436.

[106] Zabihullah, Q., Rashid, A., Akhtar, N. (2006): Ethnobotanical survey in kot Manzaray Baba valley Malakand agency, Pakistan. - Pak J Plant Sci 12(2): 115-121.

[107] Zhang, J-L., Cui, M., He, Y., Yu, H-L., Guo, D-A. (2005): Chemical fingerprint and metabolic fingerprint analysis of Danshen injection by HPLC-UV and HPLC-MS methods. - Journal of pharmaceutical and biomedical analysis 36(5): 1029-1035.

[108] Zhang, W., Guo, Y-W. (2006): Chemical studies on the constituents of the chinese medicinal herb Euphorbia helioscopia L. - Chemical and pharmaceutical bulletin 54(7): 1037-1039.

[109] Zheng, X. L., Xing, F. W. (2009): Ethnobotanical study on medicinal plants around Mt. Yinggeling, Hainan Island, China. - Journal of Ethnopharmacology 124(2): 197-210. 